Metals and Ceramics Division

\title{
THE LILARTI NEURAL NETWORK SYSTEM
}

J. D. Allen, Jr.

F. M. Schell

C. V. Dodd

Date Published: October 1992

Prepared by the

OAK RIDGE NATIONAL LABORATORY

Oak Ridge, Tennessee 37831-6285

managed by

U.S. DEPARTMENT OF ENERGY under Contract DE-AC05-84OR21400 


\section{TABLE OF CONTENTS}

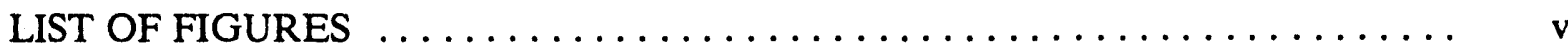

LIST OF TABLES $\ldots \ldots \ldots \ldots \ldots \ldots \ldots \ldots \ldots \ldots \ldots \ldots \ldots \ldots \ldots \ldots \ldots \ldots$

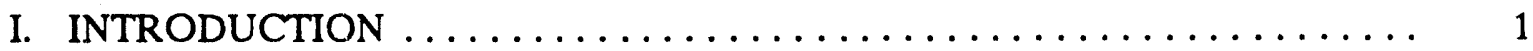

II. REVIEW OF GUIDING LILARTI PRINCIPLES AND THE EARLY

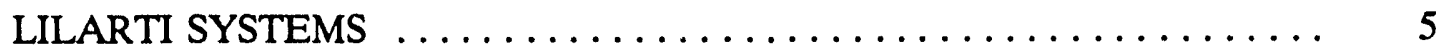

III. DESCRIPTION OF PRESENT LILARTI SYSTEM $\ldots \ldots \ldots \ldots \ldots \ldots \ldots \ldots$

IV. SYSTEM ELEMENTS ......................... 19

V. APPLICATION OF LILARTI METHOD TO NON-DESTRUCTIVE

EVALUATION $\ldots \ldots \ldots \ldots \ldots \ldots \ldots \ldots \ldots \ldots \ldots \ldots \ldots \ldots \ldots \ldots$

VI. "DIRECTIONS" FOR STUDIES $\ldots \ldots \ldots \ldots \ldots \ldots \ldots \ldots \ldots \ldots \ldots \ldots$

VII. ACKNOWLEDGMENTS $\ldots \ldots \ldots \ldots \ldots \ldots \ldots \ldots \ldots \ldots \ldots \ldots$

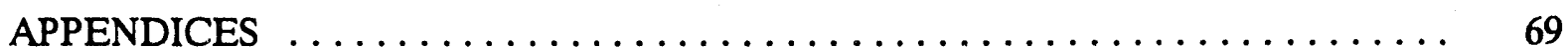

Appendix A1 - Instructions for Using LILARTI . . . . . . . . . . . 69

A1.1 - Running LILARTI . . . . . . . . . . . . . . . . . 69

A1.1a - System Utilities ............... 69

A1.1b - System Execution .............. 77

A1.2 - General Remarks on Constructing a Network-Defining Data File ............. 87

Appendix A2 - Illustrative Examples of Network-Defining Data Files . . . . . . 119

A2.1 - Source- and Target-Subsheet Parameters ......... 119

A2.2 - Periodic Connectivities .................... 127

A2.3 - Connection Distributions for Ed fe-Finding . . . . . . . 131

A2.4 - Random Connection Generation ............. 141 


\section{LIST OF FIGURES}

Fig. 1. Original LILARTI $\ldots \ldots \ldots \ldots \ldots \ldots \ldots \ldots \ldots \ldots \ldots \ldots \ldots \ldots$

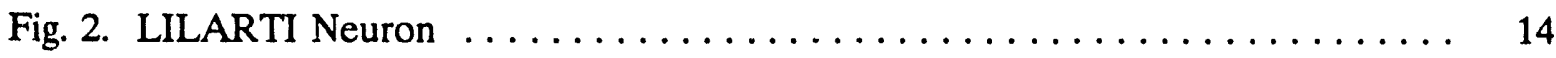

Fig. 3. Illustrative LILARTI Network $\ldots \ldots \ldots \ldots \ldots \ldots \ldots \ldots \ldots \ldots \ldots \ldots$

Fig. 4. Sigmoid response function $\ldots \ldots \ldots \ldots \ldots \ldots \ldots \ldots \ldots \ldots \ldots \ldots$

Fig. 5. $100 \mathrm{KHz}$ scan of $20 \%$ OD defect $1.1^{\prime \prime}$ from center of Fe deposit $\ldots \ldots \ldots \ldots 42$

Fig. 6. Eddy-current image decomposition scheme $\ldots \ldots \ldots \ldots \ldots \ldots \ldots \ldots \ldots$

Fig. 7. Decomposition method applied to real eddy-current signals $\ldots \ldots \ldots \ldots \ldots$. 44

Fig. 8. Steam generator tube in support $\ldots \ldots \ldots \ldots \ldots \ldots \ldots \ldots \ldots \ldots \ldots$

Fig. 9. Performance as a function of training set size: percent correctly

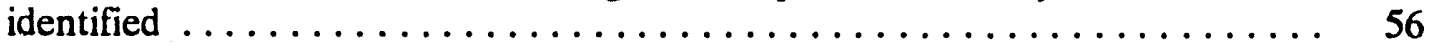

Fig. 10. Performance as a function of training set size: percent identified within $1 \mathrm{RW}$ error $\ldots \ldots \ldots \ldots \ldots \ldots \ldots \ldots \ldots \ldots \ldots$

Fig. 11. Tri-lateral question answering network $\ldots \ldots \ldots \ldots \ldots \ldots \ldots \ldots \ldots$

Fig. 12. Normal form representation of question answering network $\ldots \ldots \ldots \ldots$

Fig. 13. Example of LILARTI network $\ldots \ldots \ldots \ldots \ldots \ldots \ldots \ldots \ldots \ldots \ldots$

Fig. 14. Use of source and target subsheets $\ldots \ldots \ldots \ldots \ldots \ldots \ldots \ldots \ldots \ldots \ldots$

Fig. 15. Periodic connectivity $\ldots \ldots \ldots \ldots \ldots \ldots \ldots \ldots \ldots \ldots \ldots \ldots \ldots \ldots \ldots$

Fig. 16. Edge-finding connectivity $\ldots \ldots \ldots \ldots \ldots \ldots \ldots \ldots \ldots \ldots \ldots \ldots \ldots \ldots$ 


\section{LIST OF TABLES}

Table 1. Desirable properties of a biologically reasonable neural network ....... 6

Table 2. Desirable properties of a biologically reasonable neural network ....... 6

Table 3. Initial Goals for Neural Network-Based Eddy-Current Analysis System . . . . 41

Table 4. Trained performance for $60 \%$ training set $\ldots \ldots \ldots \ldots \ldots \ldots \ldots$

Table 5. Trained performance for $60 \%$ training set even distribution run $\ldots \ldots \ldots \quad 48$

Table 6. Trained performance for $65 \%$ training set $\ldots \ldots \ldots \ldots \ldots \ldots \ldots$

Table 7. Trained performance for $70 \%$ training set $\ldots \ldots \ldots \ldots \ldots \ldots \ldots$

Table 8. Trained performance for $75 \%$ training set $\ldots \ldots \ldots \ldots \ldots \ldots \ldots$

Table 9. Trained performance for $75 \%$ training set $(19 \times 19) \ldots \ldots \ldots \ldots \ldots \ldots$

Table 10. Trained performance for $80 \%$ training set $\ldots \ldots \ldots \ldots \ldots \ldots \ldots$

Table 11. Trained performance for $85 \%$ training set $\ldots \ldots \ldots \ldots \ldots \ldots \ldots \ldots$

Table 12. Trained performance for $90 \%$ training set $\ldots \ldots \ldots \ldots \ldots \ldots \ldots$

Table 13. Trained performance for $90 \%$ training set $(19 \times 19) \ldots \ldots \ldots \ldots \ldots$

Table 14. Trained performance for $95 \%$ training set $\ldots \ldots \ldots \ldots \ldots \ldots \ldots \ldots$

Table 15. Distribution of observed testing errors by artifact type data set (\%) $60 \ldots 52$

Table 16. Distribution of observed testing errors by artifact type

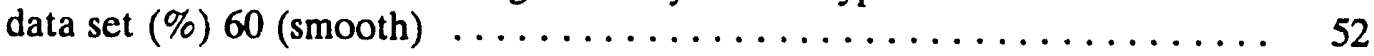

Table 17. Distribution of observed testing errors by artifact type data set (\%) $65 \ldots 52$

Table 18. Distribution of observed testing errors by artifact type data set (\%) $70 \ldots \ldots 3$

Table 19. Distribution of observed testing errors by artifact type data set (\%) $75 \ldots 53$

Table 20. Distribution of observed testing errors by artifact type

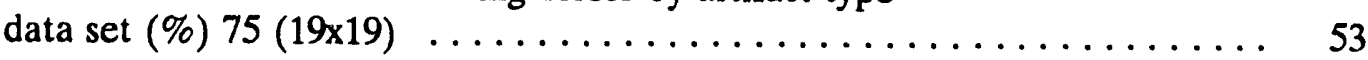

Table 21. Distribution of observed testing errors by artifact type data set (\%) $80 \ldots \ldots 4$

Table 22. Distribution of observed testing errors by artifact type data set (\%) $85 \ldots 54$ 
Table 23. Distribution of observed testing errors by artifact type data set (\%) $90 \ldots 54$

Table 24. Distribution of observed testing errors by artifact type

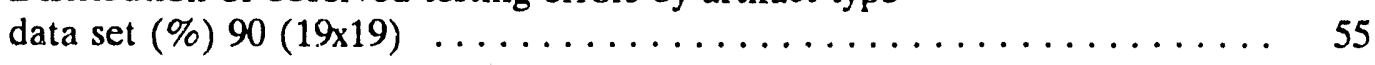

Table 25. Distribution of observed testing errors by artifact type data set (\%) $95 \ldots 55$ 


\title{
THE LILARTI NEURAL NETWORK SYSTEM*
}

\author{
J. D. Allen, Jr., F. M. Schell ${ }^{\dagger}$ \\ and C. V. Dodd
}

\begin{abstract}
The material of this Technical Memorandum is intended to provide the reader with conceptual and technical background information on the LILARTI neural network system of detail sufficient to confer an understanding of the LILARTI method as it is presently applied and to facilitate application of the method to problems beyond the scope of this document. Of particular importance in this regard are the descriptive sections (III and IV) and the Appendices which include operating instructions, partial listings of program output and data files, and network construction information.
\end{abstract}

\section{INTRODUCTION}

Over the last several years, the authors have developed a series of software-based neural network methods of increasing complexity intended (as they were initially conceived, at least) for the exploration of some of the simpler properties of one of the most complex and fascinating naturally occurring collections of neurons, the human vision system. It was recognized that at least two avenues of approach to the intended studies were open. The first, and already well worn, was avidly followed by those whose view seemed to be that if an ad hoc mathematical model could be found which performed in a manner analogous to that of some component of the human vision system, the vision system would oblige by conforming to the model. Although it cannot be denied that some very impressive systems were produced by those who took this approach, the underlying ideas were unsatisfying to others, the authors among them, whose intent was to adhere more closely to biological reality. Despite the fact that it was recognized that neither the numerical nor the biological complexity of these natural systems could be realistically modeled in presently available computing environments, to the authors a path hewed closer to identifiable biological signposts appeared to offer more promise. It is the authors' hope that the reader more comfortable with formulae will not interpret their shared preference for visual and word models as suggestive of any hostility toward mathematics. None is intended. For the authors, a direct translation of ideas into code, without the intermediate step of mathematical formalization, has seemed a way to avoid

${ }^{*}$ Research sponsored under the Seed Money program and grant (MIPR FY1457-91N5004) from the Aeronautical Systems Division of Wright-Patterson AFB.

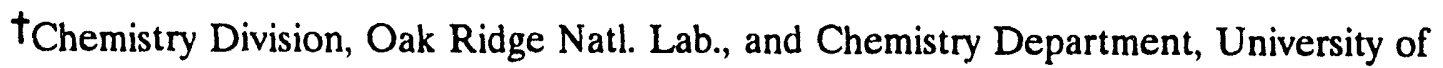
Tennessee-Knoxville. 
the too-early abstracting-away of potentially relevant characteristics. In fairness, it must be noted that, on occasion, their method has proved effective as a way to avoid the obvious!

In this document, we describe (formally where necessary, anecdotally where appropriate) some aspects of the evolution in our thinking about biological and artificial neural systems and discuss in detail the method (which bears the somewhat unfortunately chosen name, LILARTI) which has resulted therefrom. Our intent is to provide material sufficient for the reader familiar with a conventional personal computer, but not knowledgeable about neural networks, to apply our method to a problem of his own devising. We begin (Secticn II) with a review of the general principles we established at the outset as definitive of the class of artificial neural systems of particular interest to us. These are described primarily in the context of earlier LILARTI systems. The principal subject of this report, the current LILARTI methodology, is examined in Section III. Section IV is devoted to a detailed "dictionary" of the system-defining arrays and their elements. A nearly complete understanding of the workings of the LILARTI code can be acquired from a careful study of this section. A description of a very practical application of the LILARTI methodology in the area of non-destructive evaluation (NDE) comprises section V. The main body of the text concludes with section VI in which we offer some suggestions for additional studies and applications. Following the very heartfelt acknowledgments of section VII can be found two appendices which, in addition to operating instructions and a partial network output listing, include instructions for constructing both the data file representation for a simple LILARTI system and the partial file representations for a few interesting neural functions. Readers interested only in applying the method can safely skip the remainder of the introduction and all sections but the appendices, although an examination of the descriptive and dictionary sections (sections III and IV) is strongly recommended.

It is appropriate, we think, to include in this Introduction a brief history of the path by which the authors came (and almost failed to come) to be involved with the study of artificial neural networks. An interest in the general topic, which had lain dormant in two of us (Allen and Schell) for more than a decade, began to reignite during the mid eighties as every experience with other forms of artificial intelligence (most focused either on the problem of automated chemical synthesis ${ }^{1}$, hazardous chemical waste reduction ${ }^{2}$, or on the production of dedicated parallel hardware for executing very large expert systems ${ }^{3}$ ), however successful, strengthened the conviction that intelligence of the sort humans exhibit was not likely to arise algorithmically. There was no doubt that algorithm-based machines could be programmed to discover and prove theorems, reason analogically, learn in almost any domain, translate languages, extract the sense from text, and the like. There was likewise no doubt that the human brain is not, at least in any obvious sense of the term, an algorithmic engine. Yet it performs all of the noted functions, and more, and in the process endows us with a sense of self, with sentience. Because the processes of artificial neural networks represented at least a step away from the obviously algorithmic systems (if, despite the popular press, not clearly toward obviously "biological" ones), their study came to seem increasingly important to us.

As we began to consider the details of what would ultimately become the LILARTI method, we happened to encounter a delightful book by Oliver Sacks ${ }^{4}$. In a chapter entitled "The Twins", Sacks discusses brothers who, although quite retarded, exhibited a remarkable capacity for dealing in certain surprising ways with large numbers. Although they did not understand, in any conventional sense, even the simplest of arithmetical operations, they could, among 
other things, recognize, even, it would seem, generate, primes of up to 20 digits and could not only recognize the number of identical objects in a briefly displayed scene (111 spilled match sticks, for instance), but immediately produce the prime factors of the identified number. Although perhaps not as intrinsically interesting as the recognition or generation of primes, in the capacity for "N-ness" recognition, in the apparent ability to "see" and operate on numbers directly, there seemed to lie clues to the deeper operations of concept formation and concept manipulation. Because these were among the cognitive properties which we hoped ultimately to understand (however loosely they might be related to our original vision-oriented goals), $\mathrm{N}$-ness recognition suddenly came to provide a focus for our neural network musings.

Our interest in this notion of $\mathrm{N}$-ness recognition was reinforced by the discovery that very young normal children ${ }^{5}$ often exhibit some of the same capabilities so startlingly present in the "twins". Indeed, were it only in the twins that such a remarkable facility with numbers was found, one might properly have suggested that basing studies of concept formation on the behaviour of two retarded brothers bordered on retrograde science (or worse!). However, more than the capacity for recognizing $N$-ness for large $N(N>100)$ is exhibited by others than idiots savant. Not only can very young children, if they are properly instructed, perform these feats or recognition, more important, they can be taught the fundamental operations of arithmetic in the context of nothing more than the random dot-pattern flash cards with which they learn $\mathrm{N}$-ness recognition. It was, in fact, this latter point which was centrally important to what we viewed as our longer range goals for it appeared reasonable, in light of the childrens' ability to learn the operations of arithmetic only from visual exposure to random dot patterns, to expect an artificial neural network which had learned to "count" to be able, under suitable conditions, to learn to "manipulate" as well. If such a demonstration could be made, one might well argue that the rudiments of concept formation and concept manipulation were present in the network.

We appreciated the fact that it might be argued that the test subjects who displayed the capability for $\mathrm{N}$-ness recognition were simply counting very rapidly. Indeed, we recognized the triviality of constructing quite uninteresting, non-learning, neural network systems which would emulate the observed behaviour if mere counting were all that were involved. It remained our view, however, that something much deeper was at work in the young children, if not in the twins, although discovering the precise depth and nature of the operations would be rendered, in the one case by youth, in the other by retardation, a formidable proposition. Undeniably, the behaviour exhibited by the children requires considerable neural activity and a deep, if not comprehended (and perhaps, by rough analogy with the work of Julesz ${ }^{6}$ on early vision, precortical), ability to organize some aspects of patterns and numbers in something like a conceptual framework.

Two general approaches to the study of the N-ness problem suggested themselves to us. Because of a long standing interest in simulated evolution, we considered first the construction of a software "environment" in which successive generations of "genetically" constructed neural "beings" (each of which, however additionally complicated it might be, at least minimally endowed with a "retinal" sensor and an output layer by which to communicate the results of neural activity) could function and breed subject to imposed conditions tending to select for efficacy of "N-ness" recognition. We had written the necessary code and concocted a "genetically manipulatable" data file structure for neural entity representation 
before the inadequacy of laboratory computing facilities and their inaccessibility for pure research purposes were fully realized.

Confronted with this impasse, we were forced (to our good fortune, as it turned out) to consider an initially less appealing approach to the study of $\mathrm{N}$-ness. We would have, we realized, to construct, ab initio, a neural model of a significant portion of the human vision system and, by adjustment of parameters, attempt to elicit the desired behaviour. (Any reader not flabbergasted by the impertinence of the previous sentence might wish to reread it.) Brief descriptions of the working principles which guided our developmental efforts and the details of the system which resulted from them (a system, by the way, never employed for $\mathrm{N}$-ness studies) can be found in Sections II and III respectively. For the present, we draw this introduction to a close with the promised indication of the reason for our near failure to undertake neural network studies at all.

Once we had adopted the second approach to N-ness studies, it was necessary that we develop a general network model incorporating the essence, we hoped, of the biological reality to which we continued to bear allegiance. The "paper" model we produced certainly had many elements in common with the human vision system. Aside from the obviously required retina, there were feature extractors, outline or contour-producing regions, something whose function(s) bore (we optimistically asserted) some similarity to those of the lateral geniculate bodies, multiple trainable regions driven by the feature extractors, and a visual cortex. In addition, a second input sense was incorporated by which scene-describing information could be provided to the system. Last, linking the higher neural (or, as we, again optimistically, then referred to them, "cortical") levels of the two senses were very dense and trainable interconnections.

Inspection of the "paper" model suggested a variety of interesting behaviours all of which were observed, to one degree or another, when the first LILARTI was finally constructed. A working system should, for instance, be able to learn to describe unfamiliar objects and to recognize familiar objects in unfamiliar orientations. Position, scale, and rotation invariance were, in fact, observed characteristics of the early systems. Most interesting to us, it appeared that a system of the sort we envisioned, once trained in the context of a wide variety of image types, should be able to produce at its visual cortex at least a crude representation of an image for which only descriptive parameters were supplied. This behaviour was later observed as well. Because each of these model properties had a close analogue in the known behaviour of the human vision system, we were naively confident that any subsequently discovered model property would be similarly associated with reality. Thus, we were unprepared for the most startling (and, we knew, unrealistic) projection that there should be blind humans possessed of the capacity for describing and functioning in their environments despite the fact that their visual fields appeared to them entirely dark. Our unwillingness to proceed on the basis of a model which could indicate the possibility of something so preposterous led us to put off for nearly a year the construction of code based on the model. Indeed, when we did begin (principally out of desperation), it was with the understanding that we would find some mechanism for ridding the model and the associated code of this apparent offense. It was months after we had completed the first LILARTI system (for a shorthand representation of the system wiring, see Figure 1 in Section II) and had observed all the anticipated behaviours (as well as the aberrant one) that we realized that the phenomenon was well known in 
medical and psychological circles by the term "blind sight" (for a brief description see Penrose $^{7}$ ).

At this juncture, powerful working model in hand, we suffered a tragedy; Cognitive studies confronted modern laboratory reality and lost. Indeed, had there not been an application (NDE eddy-current signal evaluation) to which the image-oriented LILARTI method could readily be adapted (albeit with concomitant elimination of most of the interesting visionrelated elements), our neural network effort would have ceased. To our great good fortune, the NDE application was an interesting one and, by virtue of its own particular requirements, forced the further neural system developments which, with the exception of the historical material of Section II, form the principal focus of most of the remainder of this document.

We conclude this introduction with a brief response to the frequently asked questions concerning the origin of the name, LILARTI. Although it would please some if it were a clever acronym for the novel sequence of operations which define system behaviour, it is not. When our early considerations of potential neural network representations had matured sufficiently to permit codification in a computer program, we were confronted with the necessity of providing a moniker by which to refer to the family of programs we anticipated. "ASDFJKL;.FOR" and "QWERTY.FOR" lacked that certain essence so essential to programs for which any success is hoped and in any case were both already assigned to retiring programs on the authors' computers. Something more suggestive of the grand scope of our aspirations seemed in order, something suggestive as well of our admiration for two Arthurs, the father of the best known fictional machine intelligence, HAL, Arthur C. Clarke, and Arthur, king of Camelot. "ARTHUR.FOR" seemed a bit presumptuous a name for a system of as yet undemonstrated capabilities, indeed, for any system we could realistically envision. Even the diminutive "ARTI.FOR" anticipated capabilities whose realization lay in the distant future. The more reasonable "LITTLEARTHUR.FOR" and "LITTLEARTI.FOR" were too long for the stunted filename protocols of DOS machines. We were left with "LILARTI.FOR" (pronounced in the best Southern Tradition by analogy with Lil Abner and lilwile).

\section{REVIEW OF GUIDING LILARTI PRINCIPLES AND THE EARLY LILARTI SYSTEMS}

As suggested in the remarks of the Introduction, the authors began their neural network studies in the hope of reaching a preliminary understanding of the human vision system and of certain cognitive activities which seemed more or less directly related to it. Because a central theme of the anticipated studies would be adherence to some set of approximate biological realities, considerable effort was devoted to establishing working representations for the biological characteristics of apparent importance. These are described briefly in Tables 1 and 2 below and in somewhat more detail in the relevant material of the remainder of this section and the subsequent two sections. It should be clear that we have drawn a very important distinction between what are the essentially macroscopic properties of concern to us and the detailed processes of nerve impulse transmission, neuron firing characteristics, and the like with which any neurophysiologist would rightly concern himself. We remain, perhaps naively, 
Table 1. Desirable properties of a biologically reasonable neural network

\section{Form}

(a) Neuron characteristics should represent the behaviour (insofar as it is presently known and practicable of representation) of the naturally occurring entity. Not only the form (e.g., multiple dendrites and multiple (and distinctly functioning) axons, but the chemical properties (e.g., something like a connection altering catalyst)) should be embodied in an acceptable model.

(b) System behaviour as a function of connectivity, particularly in its tolerance for "random" variations in connection distribution in three-dimensional space, should closely represent the tolerance exhibited by natural systems.

(c) Connectivity representation should be expressed in terms of "genetic" parameters.

Table 2 Desirable properties of a biologically reasonable neural network

\section{Function}

(a) Formation of abstract representations (low level grammars) of patterns must develop naturally.

(b) There should be no necessity for a learning switch. Learning should take place in any sense, or among any set of senses, when it is neurally detected that learning is appropriate.

(c) Learning must occur without "mathematics".

(d) Connection alteration conditions and magnitudes must be determined locally, not globally.

(e) Interaction and contextual training among a multiplicity of senses or sensor inputs must be an inherent property ("cognitive complementarity") and lead to the Sormation of higher level grammars.

(f) Trained cortical regions should advance in three-dimensional space, and in neural state space, in a manner predicated on the system's training experience, but without any form of algorithmic direction.

confident that these details, although important to the sustenance of biological neural systems, are not necessarily essential ingredients of a system capable of the true cognitive activity.

Were it not for the leading parenthetical expression in Form Property Ia and the multitude of sins which may be subsumed by it, the authors would still be constructing only paper neural models, not functioning software-based neural network programs. In part because complete knowledge of neuron structure and chemistry is yet unavailable, in part because much of what 
is known is relevant only to sustaining the existence of neurons and not to their functional properties, Ia admits of less than complete realization in code. Certainly the most serious cleviation from fact involves the failure (for reasons related primarily to execution efficiency) to implement even such an obvious physical aspect of biological neural systems as multiple dendritic branching. Because of storage and computational limitations, all synaptic connections are assumed to be made on the nerve body itself. This is contrary to the known characteristic. Nevertheless, when it is necessary to do so, an approximation to the biological notion can be incorporated by the ruse of inserting additional neural nodes in existing dendritic pathways and constructing thereby a dendritic tree. Whether or not this technique even approximates the behaviour of densely branched natural systems is uncertain.

Likewise not represented in any direct manner are the chemical and electrical processes of nerve impulse generation and propagation. In fact, in our systems, as in most, neuron firing rate is represented by the magnitude of the value derived from a lookup table embodying a user-defined response function (approximately Sigmoidal in form) for neurons of the type presently under evaluation. In some of the LILARTI systems, it is arranged that the derived value for each neuron is perturbed in a quasi-random manner at times when certain conditions obtain for the relevant inputs. Not related to the "dither" process which is a key characteristic of present LILARTI systems, nor necessarily analogous to the random firing which biological neurons are known to undergo, this simplest form of random firing serves principally to suppress network behaviour characteristic only of neuron evaluation order.

A property which was, in earlier LILARTI systems, embodied (so far as we know, only by us) is the capability for "output path selection". In conventional models, output signals from a source neuron are coupled, via whatever connections presently obtain, from the source neuron "axon" to some specified and invariant set of receptor (or "Target") neurons. In those versions of the LILARTI model which included what (because of the configuration of the multiple output connections) we called "winons", a neuron could determine, on the basis of locally available and biologically reasonable information, which of two or more axonic outputs to activate in response to the signals presently impinging on the nerve body. Each of these outputs generally served a distinct set of target neurons. Neurons of the "winon" type proved especially useful in constructing the nonlearning feature extraction portions of the earlier LILARTI vision systems.

Two quasi-chemical properties have been represented in most versions of the LILARTI code. The first of these to be incorporated, the notion of a connection altering catalyst, has been the most consistently employed. Some (usually large) quantity of this material is assigned to each alterable system connection before training begins. As training proceeds, catalyst is depleted in those connections which experience weight alterations. Because the capacity of a connection to change strength or weight is made proportional to the amount of its remaining catalyst, frequently altered connections tend to "stiffen" against alteration.

The second of the two properties, referred to in those versions of the code which supported the associated operations by the term "decanting", likewise involves manipulation of connection catalyst values. The decanting process is also characterized by a removal, during training, of connection catalyst. Here, the amount removed is proportional to the signal born by the connection with the result that more frequently used connections become more rapidly 
stiffened against alteration. A not fully explored point is the interaction of these somewhat contrary behaviours.

Quite fully realized in some of the LILARTI systems is desired form property Ib. For the largest systems which we have been able to process on a 16 MByte PC (something of the order of 50,000 neurons and 300,000 connections), global system behaviour has proved to be much more a property of global connectivity patterns than of the microscopic details of individual connections. In fact, substantial local alterations can be made in the connectivity descriptions without noticeably affecting the ability of the overall system to perform its intended task. It is important to note here that the systems for which these statements are true (of which Figure 1 is fairly representative) are based on the earliest LILARTI methodology and incorporate considerable redundancy. The system of Figure 1, for instance, learns to describe images in the context of five distinct and hierarchically ordered feature classes. A disorder in any one of the feature extractors is not likely to disrupt the observed behaviour of the system. The desired tolerance against wiring perturbations is less fully realized in the more recent, and much smaller, LILARTI systems. Indeed, because position, scale, and rotation invariant recognition derive (in our systems) from the same neural regions

ORNL-DWG $92-11932$

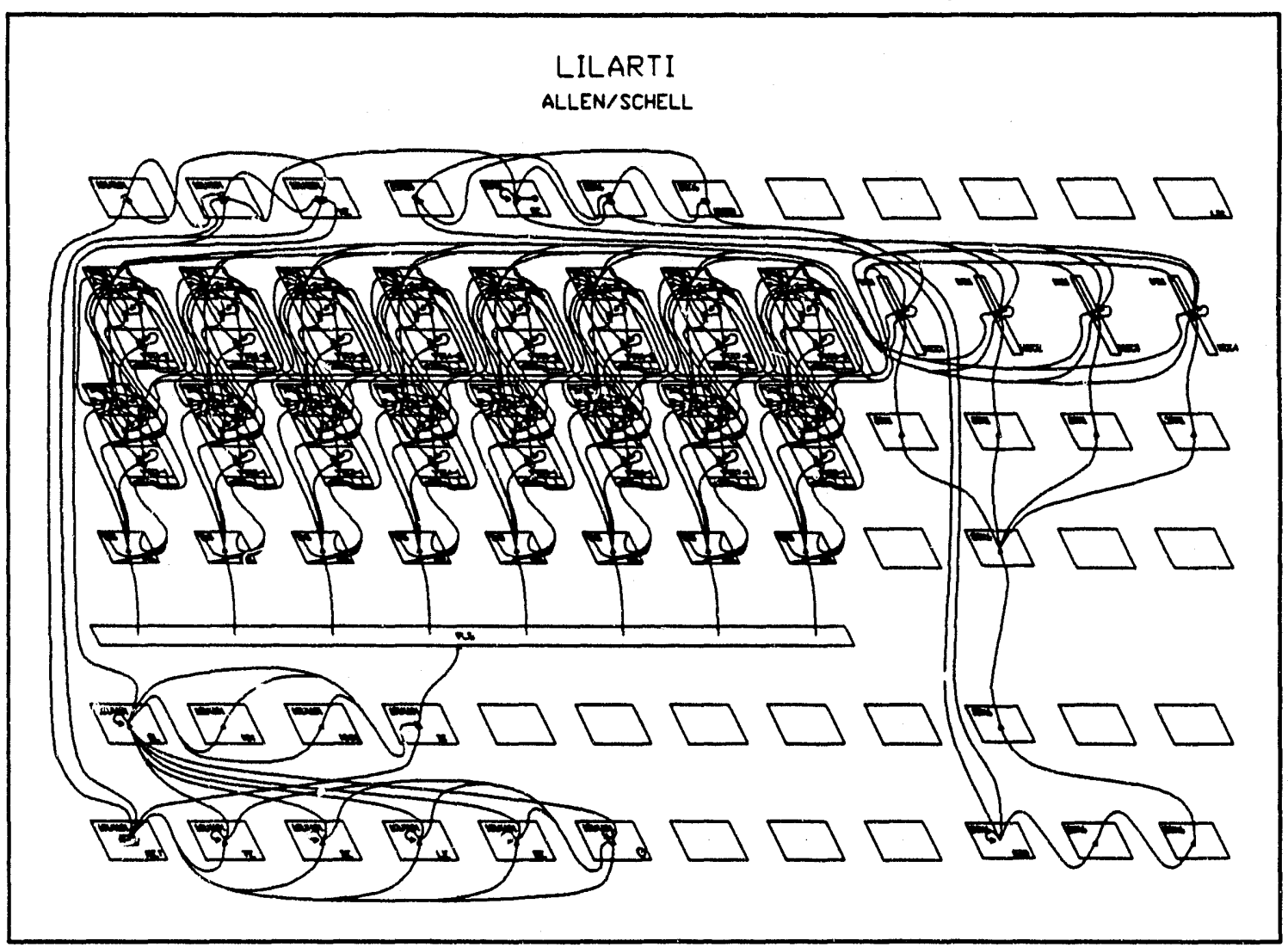

Fig. 1. Original LILARTI. 
which confer such tolerance against wiring error, no remnant of these regions is present in our current NDE-oriented systems, systems for which position, scale, and orientation sensitivity (rather than invariance) are important.

What is intended by form property Ic requires a bit of explanation. As already noted, long before the studies which resulted in our current systems were initiated, it had been anticipated that simulated evolution might prove a useful technique for exploring the emergence of certain aspects of cognition. In order to facilitate the process of "gene" combination-directed neural net formation, we adopted a file structure for network component definition whose elements, as crude analogues of genes, could easily be manipulated, combined, and recombined to direct the production of neural network "entities" of almost limitless variety. An inevitable, indeed intended, consequence of the approach was the result that specification of individual neurons or connections became almost impossible. Instead, regional properties became the objects of definition. In crude analoy to biological genetics, the file elements can be viewed as being expressed by the network construction code in something of the manner of biological gene expression. Although, to our regret, the evolution studies have, for lack of adequate computing power and laboratory interest, yet to be undertaken, the biologically oriented network description scheme, because of its considerable expressive power, has remained central to all our subsequent work.

Of more immediate practical importance than the form properties, if only because they are associated more directly with observable system behaviour, are the extents to which the above noted six functional properties have proved realizable. Again, because we have developed several variations on the original LILARTI theme and because the properties of interest represent a considerable range of difficulty of representation, realization, as the following remarks suggest, is found in widely varying degree.

Surely one of the principal problems confronting students of vision and cognition has as its focus the elucidation of the mechanism by which sensory data, i.e., an image formed on the retina of the eye, are transformed into higher level "conceptual elements" (whatever these may be) in terms of which an understanding of the environment is constructed. Indeed, there are those who would argue that it is not at all certain that such a well defined hierarchical process is involved. Even were the favourable evidence conclusive, the question of the distribution of the associated subprocesses among "trainable" and "un-trainable" neural regions remains an unresolved issue. Although we adopted the hierarchical process view, we recognized the necessity of exploring a variety of distributions for the relevant subprocesses. Thus, the very earliest regions of our artificial vision systems were "pre-wired" for specific tasks (linedetection, edge-detection, vertex-detection, etc.), whereas progressively higher regions were successively less pre-ordered. Because frequently encountered combinations of the preordained visual primitives (and successively higher level combinations of combinations, etc.) appeared to give rise to increasingly abstract, trained, representations in the higher neural levels, requirement Ila was, in our very early systems, at least primitively, supported.

Two points bear mention here. First, the behaviours noted above were sought for and observed only in the earliest, specifically vision-oriented LILARTI systems. More recentiy developed systems, as noted elsewhere, have been carefully contrived for specific applications and have incorporated none of the low-level feature extraction wiring. Perhaps more significant is the fact that any beginning student of neurophysiology could enumerate vision 
functions not even considered for incorporation in our models. A very important example is stereo-vision. Our elimination of this from consideration was motivated principally by the conviction (bourn out to a degree by the work of Julesz, op.cit.) that the processes involved were of relatively low level and thus not central to the problem of scene understanding. It should be admitted that we then had no model for the neural processes which might be involved! Among other omitted properties was a facility for temporal recall of image sequences. Neither were color vision and the associated problems considered. Image segmentation, the ability to identify the portions of an image which "belong together" was likewise not incorporated in any direct manner. Nevertheless, despite these and many other limitations, the intended behaviours (position, scale, and rotation invariant image description) were observed in those systems which included the necessary low level wiring.

The focus for one of the more obvious disparities between natural and artificial neural systems is the clearly drawn distinction generally associated with the latter between "learning" and "non-learning" behaviour. Although there are certainly times and circumstances which lend themselves more felicitously to the processes of human learning, there is certainly no distinction so evident in biological systems that the notion of a "learning switch" could have validity. Nevertheless, except for ours, all artificial neural systems of which we are aware possess something equivalent. Our systems, when confronted with the unfamiliar, determine, in a purely neural manner, whether or not there is sufficient descriptive information in the current environment to make "disambiguation" possible. If such information obtains, the system attempts incorporation into the existing state configuration. If not, the network is forced to respond with the equivalent of "I don't know". Thus, Function property IIb is supported in one way or another in all our systems.

The mechanism by which learning takes place represents another clear, but often blithely ignored, distinction between natural and artificial neural systems. Embodied in the better known conventional methods are mathematical schemes of considerable sophistication. Although it cannot be argued that these do not produce the desired trained states of the artificial neural systems to which they are applied or that they lack great mathematical elegance, it is most assuredly the case that biological neural systems do not possess the computational apparatus with which to carry out the operations required for their support. Furthermore, the connectivity of natural neural systems is not adequately modelled by these mathematical schemes to justify any assertion of relevance. Because we hoped to contrive a learning scheme predicated on the biologically reasonable, we eliminated these more mathematical methods from consideration from the beginning. Whether or not the assumptions which we have made about training-related neural functioning (see Section III for a description of the latest scheme) represent a closer approximation to reality is a mat er we presently leave the more physiologically trained reader.

Closely related to the question of learning mechanism is that of the extent to which training at one location is determined by actions at others more or less distant from it. In several of the "mathematical" schemes, quite unrealistic assumptions are forwarded about the range of connectivity and about the extent to which the states of neurons and connections distant from, and not directly connected to, those for which training is being considered can exert influence on that training. In the various versions of the LILARTI models, training influences have been of two general kinds. In the simplest of the systems, alteration of the strength of a connection weight is dependent only on the states (a term which, even in the early LILARTI 
models, conveyed more than firing rate information) of neurons at opposite ends of a synap ic junction and on the present characteristics of the synaptic junction itself. Because the neural elements (the "winons" and "nuevons") of these earliest systems incorporated multiple, and multiply functioning, outputs and inputs by which state information could be communicated among neighbouring (but not necessarily trainably connected) neurons, and because this ancillary state information was used to influence local training, the associated training rules did not occupy any of the classical niches. In more recent systems, each neuron is forced to direct the alteration of input connections in a way which the neuron has "discovered" minimizes (subject to a concomitant minimization of the energy required for the alteration) an error signal developed by the neuron in response to an operation we call "dithering".

A proper description of what we mean by the term "cognitive complementarity" and the route by which we came to recognize it as an essential ingredient of any realistic neural model lies beyond the scope of these brief notes. Nevertheless, it is important to understand its principal implication, namely, that training in response to sensory input of any one type must occur in the context of the evolving responses emerging as a result of such stimuli as are present at all other sensory inputs. Thus, in our earliest systems, visual training, for example, does not occur only in the context of visual stimuli, but in response as well to any other stimuli which, via any other sense, are (at least approximately) simultaneously present. It is this "mutuality of context" aspect of training which, we assert, supports the development of increasingly high levels of the abstracting grammars in terms of which the system learns to describe the inputs with which it is presented. Associated with the notion of cognitive complementarity is the capacity for constructing a "mental image" from a presented description. Almost certainly, it is a mechanism of this sort which is responsible, in the human, for the phenomenon referred to earlier (and popularly known) as "blind sight" which exhibits itself in some blind persons as the startling capacity for describing and functioning in their environments despite the fact that their visual fields are entirely dark.

It is with requirement $\mathrm{II}(\mathrm{f})$ that the connection with biological reality is perhaps most tenuous. It is not, to our knowledge, convincingly demonstrated that there is anything resembling a regular advance of some "front" which represents the boundary between fully trained and as yet not fully trained regions of the various human cortexes. Neither do we argue that such regularity obtains. Certainly, however, it is widely assumed that the "plasticity" or trainability of individual synapses decreases with time (and, it is further assumed, with the training which has taken place during that time). If training is, by the continuing appearance of stimuli, to continue "beyond" a non-plastic region, some provision must surely be made for associating such responses as this region does make (however predicated they may be on the effects of previous training) with still alterable responses in other, but connected, cortical regions. It is most certainly the case that, if something of this sort does occur, it can require no formal mathematics for its realization. It was for the facilitation of this assumed behaviour that the new class whose elements we christened (to our great and eternal regret!) "winons" was introduced.

A characteristic not thus far addressed, the capacity for learning (and recalling) in a temporal context, although not particularly relevant to the manipulation of "static" images, nevertheless should be of concern to those considering the construction of biologically reasonable artificial neural system. Humans, after all, do not hum all the notes of a favorite musical theme at precisely the same instant (although, given the nature of what passes for contemporary music, 
such an action would represent a considerable refinement!). Neither do they recall events without some sense of temporal ordering (despite the efforts of political reconstructionists). Among the conventional schemes, perhaps the most computationally reasonable (yet, at least for recall over long spans of time, biologically unlikely), are those which, by one means or another, provide for feedback from later to earlier stages of the neural system. The intent is to make subsequent system responses contingent on present and/or prior stimuli. Although we would agree that, in some large sense, this notion seems supportive of the observed behaviour, we think it unlikely that so direct and simple a feedback mechanism can be responsible for processes as disparate as the proper ordering of words in a seritence and the correctly ordered recall of historical events. The very different direction of our own initial efforts in this connection will be the subject of a subsequent note.

We would be remiss were we not to acknowledge the enormous body of neural knowledge which, with unintended high hand, we necessarily ignored. A quick survey of even the simplest text on the neural processes of vision ${ }^{8}$ would elicit a list of impressive proportion. The very important details of neuron pulse production, latency, pre-somatic inhibition, the diversity of neuron form and function, and many others were of necessity suppressed. We leave the reader to decide whether or not the observed system behaviour justifies our choices of represented forms and functions and our assertion of biological relevance.

\section{DESCRIPTION OF PRESENT LILARTI SYSTEM}

In one way or another, all the earlier LILARTI systems were designed to avoid the necessity of confronting directly a single vexing issue arising from the extended, non-planar, distribution of neurons and neural interconnections which any realistic model would embody. The issue in question was the difficulty of concocting reasonable training rules for those system connections (of which, in extended systems, there could be many) having neither terminus at either an external input node or a network output node. As very briefly suggested in the previous section, our initial solution was to design neurons equipped with specially functioning inputs and outputs by which the neurons could probe their local environments and respond by directing training of the associated connections according to the simplest of Hebbian rules. By such ruses we were able to retain relative simplicity in the relevant computational processes and thereby to sustain acceptable performance in a PC environment. We came to realize, however, that the trained network performance, however impressive or (as we then thought) biologically reasonable, was, on a per neuron or per connection basis, not as substantial as we might have wished for. The NDE studies, about which we shall have more to say, required neural systems of as many as 5000 neurons and 300,000 connections. The nagging suspicion that these figures were larger than they need be coupled with the increasing awareness that we were "inventing" rather than modeling biological reality continued to alert us to the necessity of coming to better terms with the goals we had originally set.

As we began to confront the necessity of a serious reconstruction of the LILARTI method we recognized that we could succeed only by coming at last to terms with the problem we had so far so adroitly avoided. We would, we knew, have to devise some realistic mechanism by which "internal" neurons (those not directly connected to external inputs or to network outputs) could direct the alteration of their associated connections in some reasonable 
response to cues originating at potentially distant neural sites. We were aware of such classical solutions to the problem as the widely employed "back-propagation of error"10 scheme and were determined to avoid something which appeared to us so devoid of biological motivation. Nevertheless, it was clear that, for systems of the sort so convenient for image classification and feature extraction (the issues of importance to the then current NDE problem) provision would have to be made for training throughout the entire network to respond in some coherent manner both to current input signals and to detected errors in the output signals. We had to discover, if this process were to be at all consistent with our biologically oriented goals, how this might be effected by neural mechanisms for which there existed some evidence.

We sought the realization of yet another goal, one which biological neural systems appeared to achieve with ease. We intended to require of the proposed training scheme that each new training episode do minimal damage to such trained-for states as might already exist in the network. Satisfying this requirement, it appeared, would depend upon the ability to devise what in effect would be a cost function, a measure of the "work" required to effect training, whose value would be minimized in a manner consistent with the performing of the indicated training.

As we speculated on the proper form for a new training scheme, we found ourselves frequently reminded of a so far much under-employed neural property (yet one on whose reality we had initially insisted), the quasi-random pulse production (or firing), which naturally occurring neurons experience. Might this property, we began to wonder, be used to advantage in support of a more biologically reasonable training scheme? Although we had incorporated the concept of quasi-random neural firing in all our earlier systems, we had intended it primarily as a device for inhibiting training processes based only on neuron evaluation order or system geometry. It occurred to us after too-long deliberation that the process might be employed in a very different and much more important role. We will shortly describe the incorporation of this nearly overlooked property into the new LILARTI scheme. First, it is necessary to describe the characteristics of the neurons which populate neural systems constructed according to its principles.

For the reader not familiar with the characteristics of typical synthetic neurons we can safely note only that the vast majority are representable as two terminal non-linear devices for which some transfer function (frequently, but not necessarily, a Sigmoid) relates the sum of the signals appearing at the "input" terminal to the value of the signal which will be caused to appear at the "output" terminal. This latter is assumed to represent the "fining rate" of the neuron in question. For almost all synthetic neurons, those of LILARTI systems included, there is some input signal (denoted by the variable ONSET in the LILARTI systems) below which there will be no output and an input signal (denoted by the variable SATVAL in the LILARTI systems) above which the output will not increase beyond some saturation value. For the LILARTI systems, all neural outputs are treated as integers lying in the range between zero and some sheet-dependent maximum value (generally 1000 ).

In order to support (among other things) the intended training mode as well as the requirement that there be no "learning switch" in the new LILARTI system, a neuron of very different form was required. As suggested by the sketch of Figure 2, the new neural entity is characterized, not by two, but by nine terminals (four of which, RTE, PSIE, DDF, and STQ 
are, because connections could not terminate at them, virtual). Of the three "real" input terminals (EXT, I0, POIN), the EXT and POIN nodes will be most familiar to those acquainted with classical neural networks. The POIN (or, post-synaptic input) terminal is completely equivalent to the summing input node for trainable connections found in almost all other artificial neural systems. It is at this node that the connection-weighted outputs of other system neurons are combined into the single input value which, via the Sigmoid transfer function, will determine the neuron output value. It is this value which appears at the $\mathrm{N}$ (for Normal) output terminal. The EXT input terminal supports non-trainable inputs arriving from external sensors like the LILARTI retina and the so-called "Keyboard" input. In the current version of the LILARTI code, the management of EXT signals is entirely algorithmic, the node serving, for each neuron, as a convenient storage location in memory. Thus, no inter-neural connections link any neural outputs to an EXT terminal.

The 10 input provides, via generally untrainable connections, a mechanism for accepting what are referred to in the LILARTI system as External Inter-Neural Input signals. These signals represent the outputs of the upper levels of the various "sensory" regions and, via the 10 connections, link the upper levels of the all the system "senses". Signals of this type, when present, are used by the receiving neurons to determine the signal levels at the associated DN (for Desired N) terminals. We anticipate subsequent remarks a bit by noting that each neuron in a LILARTI network of the new type functions in such a way as to force the signal appearing at the $\mathrm{N}$ terminal to equal (within some system-defined limit) that at the DN terminal (however this latter is determined). It is worth noting that the term "desired output" (or something like

ORNL-DWG 92-11933 it) has come, quite independently of our own work, to represent an analogous property in conventional back-propagation systems.

Although the functions of the four virtual terminals will become evident when we take up the details of system training behaviour, it may be of use to describe them briefly here. Thus, PSIE (post-synaptic input error) is a measure of the disparity between the input presently obtaining at the POIN terminal and that input which would be required in order to sustain the $N$ output at a value equal to the current DN output value. RTE (received target error) is the sum, at any neuron " $n$ ", of the error signals (about which more subsequently) generated at the target neurons for which $\mathrm{n}$ is a source. STQ (source training quality) was intended as an indicator of the degree to which a neuron had succeeded in bringing its $\mathrm{N}$ output into equality with the DN signal. Although in early versions of the code this parameter assumed values in the range 0.0 to 1.0 and modified learning rates proportionately, it presently is allowed only flag status ( 0 or 1 ) and signifies simply that the associated neuron has not $(0)$ or has (1) attempted to establish a desired output value. DDF (dither-done flag) denotes the status of 
the "dither" operation by which a neuron (specifically, a neuron bearing no I0 type connections) begins the process of determining the value for its DN output.

It will prove convenient to consider LILARTI behaviour in the context of the specific network example illustrated by Figure 3. The indicated network includes an input neural level (or, in the presently popular vernacular, layer or slab) (perhaps a "retina"), two intermediate levels, and an output level. Although each level is in this case linked by trainable connections only to the immediately superior level, it is important to note that, in contradistinction to the usually obtaining condition in back-propagation systems, the connectivity is relativity sparse. Thus, it is specifically not the case that each neuron at a lower level is connected to all neurons on the superior level (although the inclusion of such extensive connectivity is not forbidden). Moreover, each neuron on an intermediate level may be connected (trainably or otherwise) to arbitrarily many neurons on the same or any other levels (although, for the intra-sheet connectivity at the intermediate levels, the more general situation is to provide only for nearest-neighbour lateral inhibition). In order to simplify the following discussion, we include in the example network neurons serving only one "sense". The I0 connections serving the inputs at the top level of this sense are assumed to originate at the $\mathrm{N}$ outputs of the top level of one of the complementary senses not illustrated here.

We begin our discussion of system operation by examining the response of the system to some set of signals (representing, in the illustrated example, the pattern " $A$ ") applied to the EXT input terminals of the lowest (or "retinal") level "H". The appearance of these signals results immediately in the setting of the $\mathrm{N}$ and DN outputs of those retinal neurons for which EXT signals obtain to values defined by the Sigmoid function associated with the retinal level. Since, by definition, $\mathrm{N}$ and DN outputs are equal for neurons bearing only EXT input signals, the corresponding STQ values are simultaneously set to unity. Now, via whatever connections link the retinal level with the POIN inputs of the first intermediate level "I", signals appear at "I" and, in the context ORNL-DWG 92-11934 of the associated Sigmoid function (which, note, need not be identical to the retinal function or to any other function in the system) result in output signals at the $\mathrm{N}$ terminals of the level I neurons. Because the neurons on "I" have yet had no opportunity to attempt determination of the $\mathrm{DN}$ values, these remain at whatever values might previously have obtained. Until the DN values are determined, the STQ values are set to zero. By the same processes, signals are communicated to the second intermediate level "J" and lead to the setting of $\mathrm{N}$ and STQ terminal values there.

It is only with the appearance of signals at the POIN inputs of the top level that additional processes may occur for it is here that I0 signals may be present and here that the disparity between the DN outputs they produce may be assessed in the context of the $\mathrm{N}$ out-puts resulting from the POIN inputs. Two general conditions may obtain. Either I0 signals will be 
present or they will not. If they are not, system operation ceases. If they are, either the corresponding DN outputs will match (to within some specified system limit) the $\mathrm{N}$ out-puts for all top level neurons or they will not. If they do, operation ceases. To this point, the general behaviour of a LILARTI system is quite similar to that of a classical back-propagation system. It is the set of error-reducing processes initiated if any $\mathbf{N}$ fails to match the corresponding DN which distinguishes the LILARTI method. These processes, which comprise network training, are described in the next paragraphs. In the interest of simplicity of explanation, we shall consider only the processes initiated by one DN/N disparity at the top level and shall trust the reader to appreciate that it is the superposition of many such processes which constitute full system behaviour.

We suppose that a POIN signal has finally reached some neuron " $\mathrm{n}$ " at the top level " $\mathrm{K}$ ", a level on which there is at least one neuron, not necessarily $n$, for which an 10 input signal is present (a condition readily determined neurally or, as is the case with the present LILARTI code, algorithmically). Given these conditions, the POIN signal at $n$ is compared with the associated 10 signal. If the disparity is smaller than some system limit, conditions at $n$ need be evaluated no further. If it is greater, the difference between I0 and POIN is stored at $\mathrm{n}$ as the "post-synaptic input error" (PSIE). When all the neurons on $\mathrm{K}$ have been examined for post-synaptic input error, a signal is generated which is broadcast to all levels serving as sources for $\mathrm{K}$ (here the single level $\mathrm{J}$ ). In general, for every neural sheet at which this signal is received, the next phase of the training process begins. It is during this phase, which is repeated for every successive "inferior", trainably driven, level, that the process we call "dithering" occurs. The reader will recall that we earlier hinted that the random firing so characteristic of naturally occurring neurons might in fact provide the means whereby trainably connected neurons could determine their optimum firing rates. We describe next how this rate-determination process is implemented in the current LILARTI system.

Upon receiving, at some inferior level ( $\mathrm{J}$ in the illustrated example), the indication from a superior level that the input comparison process has been completed there, there is initiated the process which is intended to model the random firing characteristic of biological neurons. For each neuron " $\mathrm{m}$ " on the inferior level, the following sequence of events occurs. First, the $\mathrm{N}$ output of $\mathrm{m}$ is fixed at its current value while processing for the entire network continues for a few more iterations. During the last of these iterations the PSIE signals at the neurons for which $\mathrm{m}$ is a source are evaluated in the context of the weights linking $\mathrm{m}$ and the targets. The result of this evaluation is combined with the pre-synaptic inputs presently obtaining at $\mathrm{m}$ to produce a measure (the "received target error" or RTE) of the "work" which would be required to reduce the net target PSIE to zero. Next, with the output of $m$ fixed at a value slightly above its current output, the just noted process is repeated and a second value is determined for the RTE. Finally, the process is repeated with the output fixed at a value slightly below the current $m$ output and a third value for RTE is determined. From these three values of RTE an estimate is made of the output value which $m$ should have in order to minimize the total PSIE of the target neurons which $\mathrm{m}$ serves as source. Two operations now follow. The DN output terminal of $m$ is set to this estimated value and a PSIE value is computed for $m$ reflecting the error between the presently obtaining POIN value and the value which would be required in order to produce the just set DN output. Note that no connections have been altered to this point. When the operations associated with this "dithering" process have been completed for all neurons at level $\mathrm{J}$, signals so indicating are broadcast to all levels serving as sources for $\mathrm{J}$ (here the single level $\mathrm{I}$ ) and the just described dithering phase begins for all receiving neurons. 
It is only when the notification process encounters a level for which the STQ values are already non-zero that something different occurs. In the illustrated example this situation obtains at level "H" (the retina) since, as already noted, neurons for which only EXT input signals appear immediately assume equal $\mathrm{N}$ and $\mathrm{DN}$ outputs whose values unambiguously reflect the input signal values.

To this point, one may imagine that a "wave" of dithering and DN output determination has propagated backwards from the highest, I0-bearing, levels to the lowest, EXT-bearing, levels. Such a wave might reasonably be expected to traverse the dendritic tree in biological systems. The processes initiated by the encounter between this wave and an EXT-bearing level may also be viewed as a wave, a wave whose actions involve (at last) the alteration of connection weights and the setting of STQ values. Thus, the neurons on the lowest intermediate level (I in the example), having detected "good" sources, attempt to drive their $\mathrm{N}$ outputs toward the previously established DN values by directing the alteration of those connections which link them to active retinal source neurons. The magnitude of the weight alteration process for any neuron depends, by simple proportion, upon the contribution which a change in its output will make to reduction of the received target error.

As each neuron in turn completes the associated input weight alteration process, a new value is computed and set for the $\mathrm{N}$ output. In earlier versions of LILARTI, the disparity between this new $\mathrm{N}$ and the current DN was used in the computation of an STQ value. In more recent editions, the STQ output for a neuron serves only as a flag signifying that DN determination is complete. When all STQ values have been set, the next superior level detects the fact (again, upon receiving the above noted wave) and the just noted processes of weight alteration, $\mathbf{N}$ computation, and STQ setting continue there. This forward wave of processes finally stops when it encounters a level bearing $I 0$ inputs (i.e., the level or levels at which the initial wave began). If, after the weight alteration process is complete for this top level, the disparity between POIN and 10 signals has been reduced sufficiently, system operation ceases. If it has not, a new backward wave of dithering and DN output determination begins.

Our description of LILARTI network operation has thus far concentrated on the relatively microscopic details of network behaviour in the context of one presented image. Since, for any imaginable application, a network must be capable of responding accurately to many images or stimulus sets, some mechanism must be provided for training and for confirming network responses in the wider context of multi-element training sets. We conclude this section with descriptions of the two functions which support these operations in the current LILARTI. Because it most resembles the process employed in the preparation of more familiar neural network systems, we begin with a description of the training mode referred to in the code by the rather obvious term TRAIN. We complete the discussion of training mechanisms with a few remarks concerning the more interesting TUTOR mode.

The TRAIN mode can be best described as a brute-force method of system training, one, moreover, completely devoid of subtlety. The user selects the elements of a training set (by methods described elsewhere and not important here), establishes values for a few training parameters (number of training cycles, number of training attempts per image per training cycle, training-complete recognition scheme, and the like) and starts the system. Of the parameters, only the training-complete recognition scheme requires comment. One of the utility subroutines in the LILARTI code, RECOGNIZE, serves to identify the image (if any) corresponding to the current network response. Because RECOGNIZE concerns itself only 
with the dominant neurons comprising the overall network response it is (by design) insensitive to specific neural outputs and, thus, insensitive to errors which may obtain between 10 inputs and corresponding POIN signal values. Thus, when the user selects the trainingcomplete scheme involving RECOGNIZE, training for an image is deemed satisfactory when the dominant network response is identified as properly corresponding to it. Because full convergence of POIN and IO input values is not required the training process can be completed relatively quickly. If, on the other hand, the user elects (by choosing not to invoke RECOGNIZE) to force full convergence, the training process will proceed more slowly. We have not completed an evaluation of the relative benefits of these two alternatives and can only comment that it appears that the superiority of either depends upon the nature of the training images. In either case, for each training cycle (i.e., for each complete sweep over the training set) the order of image presentation is randomized so as to reduce the probability of training for artifacts related only to presentation order.

Whether or not the RECOGNIZE-based training-complete scheme is invoked, the RECOGNIZE utility is employed during network preprocessing (the network operations performed for determination of the requirement for training) for both the TRAIN and TUTOR modes of network preparation. During preprocessing it serves (in concert with the routine COMPUTE_IMAGE_SIMILARITY) to suppress training for images insignificantly different from already known ones described by substantially different parameter values. Failure to take this precaution would have the effect of forcing the network to learn, for instance, the equivalent of $A=4$ and $A=10$. Although it is certainly possible to provide for the situation in which multiple and distinct responses to a single stimulus are possible (indeed, such behaviours are more interesting and typical of reality than the monotonic response characteristic forced by RECOGNIZE), the applications to which the LILARTI systems have so far been applied depend upon a unique correspondence between stimulus and response.

The TUTOR training mode differs in several respects from the TRAIN mode. In its earliest realization, the most obvious of these was the division of training material, whatever its nature, into ordered groups of similar, or sensibly related, things. Training was performed for each group, in order of increasing group "difficulty", until "comprehension" obtained. Further, an attempt was made to capture the behaviour of an effective human tutor who, recognizing a difficulty arising during the training of a student, gives such additional attention to the problem as is required for clarification. A good tutor, moreover, constantly reviews earlier learned material to ensure not only that it is retained, but that it is being assimilated in the context of current material and that new material is expressed in terms of earlier-learned "primitives".

Although something of the flavor of each of the above noted characteristics was captured in the original TUTOR training mode, present applications require suppression of some of the features (although they can still be invoked when required). Thus, the individual training groups, once populated with closely related elements, are filled with members randomly selected from the complete training set. Training proceeds one group at a time, advancing to the next group only when satisfactory trained performance obtains for all earlier groups. Training is complete when the TUTOR can make a complete pass over all groups, testing performance on each group element, without discovering that additional training is required. The process is illustrated in detail in Appendix A1.1.

What, one must surely ask in conclusion, has been gained by adopting the complex and computation intensive dither-based neural processing scheme (a scheme to which we choose 
to give the somewhat descriptive name, bi-systolic)? At the very least, the efficiency of the new approach is such that we have been able to duplicate older results which required neural systems of several thousand trainably connected neurons with a system comprising (excluding the still present retina) fewer than one hundred. This advantage has not been won without a price, however. The relaxation mechanism associated with the dithering operation requires that, for each of the dithered neurons, the complete neural network be processed for four or more additional iterations (the number depending on the range of connectivity to which the dithering is expected to be sensitive) in order to ascertain the new DN output values. Thus, the overall speed, although substantially greater, by virtue of the smaller number of neurons, than that exhibited by our earlier systems, is not as much greater as the ratio of neuron or connection counts would suggest.

The advantage which, if indeed realized, we consider most important is one for which, for lack of time, we are as yet unable to provide unassailable evidence. Our choice of the form for the Received Target Error was predicated on the desire to force, as nearly as such could be realized, non-destructive training behaviour and thus to avoid the situation so commonly encountered in other artificial neural systems in which each newly trained-for example may require retraining for all previously learned examples. By defining Received Target Error as we did (specifically, by including a crude and, indeed, heuristic estimate of state-distorting "work" in the expression), we hoped to provide for a training mode in which already trainedfor neural states would be minimally perturbed by new training and would instead be, at least to some degree, incorporated in the state representations of newly acquired material. For the present we can but note two points in support of our contention that the hoped-for advantage has been realized. First, the new system learns training sets of a given size eight to ten times faster (and, by implication, with concomitantly fewer retraining cycles) than any previous version of LILARTI. Second, training sets so large that they were unlearnable by older LILARTI systems are readily acquired by the new. Indeed, for certain data set types, the new LILARTI can learn as many as three times the number of examples of which the older systems were capable. Nevertheless, as tempting as it may be to accept these points as proof of the realization of the fundamental goal, they are merely suggestive. Only with much more evaluation and theoretical study of the new LILARTI method can the assertion be accepted as demonstrated.

An advantage which is apparent only in the longer view derives from the ease with which the new method could be incorporated in one or another dedicated VLSI parallel processing implementation. Preliminary designs for both a very simple two-bus synchronous system and a more complex, but potentially more powerful, asynchronous scheme have been developed.

\section{SYSTEM ELEMENTS}

The material of this section is based on the "Dictionary" listing appearing at the beginning of the FORTRAN source code and is intended to define the more important aspects of the present LILARTI system in terms of the principal constitutive arrays. The ruling consideration in choosing the order in which to describe these arrays was the desire to illuminate the structure of a typical LILARTI configuration. Thus, we begin with the four arrays whose elements are filled upon the reading of a user-generated system-defining file. We shall have much more to say about the entries in these first four arrays in those appendices in which we take up the issue of generating system-defining files. Of these four 
the fundamental is the array, LEVELPARM, which defines the simplest properties of the sheets of neurons from which any LILARTI system is constructed. Here, and throughout this section, various forms of the indices $\mathrm{K}, \mathrm{N}, \mathrm{I}, \mathrm{J}$ appear. $\mathrm{K}$ and $\mathrm{N}$ define the spatial coordinates of the referenced neural sheet, $I$ and $J$ the coordinates of a neuron on a sheet. $K$ denotes the vertical position (or "Level") of a neural sheet, $\mathrm{N}$ its horizontal position. In all cases, a neural sheet origin is taken to be at location $I=1, J=1$. The suffixes $S$ and $T$ (as in $K S$ and $K T$ ) denote, where required, "Source" and "Target" where Source and Target refer respectively to neurons on the pre-synaptic and post-synaptic sides of a synaptic junction. The fact that the present code does not support three-dimensional distributions of neural sheets has posed no difficulties. As a point of warning, it should be noted that the terms "level" and "sheet" occur throughout in two (generally recognizable) contexts. Used together (as in "level/sheet") they define the coordinates of a neural region. Used separately, either may refer to an already referenced region. In this context, the referencing mechanism is usually in the form of a parenthesized pair, as in $(1,1)$, wherein the first element refers to the level (or vertical) coordinate, the second to the sheet (or horizontal) coordinate.

For any neuron-bearing region defined by the pair $(\mathrm{K}, \mathrm{N})$, the following definitions obtain for the LEVELPARM elements:

$\operatorname{LEVELPARM}(1, \mathrm{~K}, \mathrm{~N})$

Total Fanin (from all source sheets) for a typical neuron on $(\mathrm{K}, \mathrm{N})$

LEVELPARM $(2, \mathrm{~K}, \mathrm{~N})$

Output Limit for a typical neuron on $(\mathrm{K}, \mathrm{N})$

LEVELPARM $(3, \mathrm{~K}, \mathrm{~N})$

Depth (I) of $(K, N)$

LEVELPARM $(4, \mathrm{~K}, \mathrm{~N})$

Width $(\mathrm{J})$ of $(\mathrm{K}, \mathrm{N})$

For convenience, sheet 1 of level 1 (or, as warned of above, sheet $(1,1)$ ) is defined within the program body as the "retina". The input sheet for descriptive parameters (the so-called "keyboard input") is defined by the program variables KBD and NBD and is usually placed at $\mathrm{K}=1, \mathrm{~N}=10$. Other "sensor inputs" are generally implemented on sheets at level 1 as well. Neural sheets defining system output (of which there are as many as there are of the above noted descriptive parameters) are, by convention, distributed at level 6 beginning with sheet 9 although they may be re-distributed as the user prefers by adjustment of the entries in the array KNCOMP.

Neural sheets not otherwise deployed at one of the above noted positions may be distributed at the convenience of the system constructor throughout the region specified by the programestablished limits KCORTSYS and NCORTSYS. For the present code, these have the values 6 and 12 respectively. The corresponding variables for sheet size system limits (ICORTSYS and JCORTSYS) are presently fixed at a value of 81 . Note that, although sheets are usually "square", they may in general be rectangular. 
Two arrays, CORTPARM and CONNPARM, are required for specification of the connections which link neurons distributed on the sheets defined by LEVELPARM. The former stores parameters applicable to all connections linking specific sheet pairs. The latter defines, in terms of "connection classes", the spatial distribution, initial weight, etc. for groups of connections linking specific sheet pairs. Because the functions of the various CONNPARM entries will be easier to grasp in the context of the CORTPARM definitions, we describe the latter first.

In the following description of the CORTPARM array elements, the variables KS and NS will define the position of a "Pre-Synaptic" (or "Source") sheet, KT and NT that of a "PostSynaptic" (or "Target") sheet. For any (KS,NS) and (KT,NT), the following definitions obtain:

\section{CORTPARM(1,KS,NS,KT,NT)}

Total Fanin for a typical neuron on (KT,NT) with respect to source neurons on (KS,NS). Values for this parameter are computed during network construction from the values appearing in CONNPARM and represent the sum over all connection classes (about which more subsequently). The sum for all (KS,NS) pairs for a common (KT,NT) pair yields the entry in LEVELPARM(1,KT,NT). It is important to note that the very general connection generation process permits construction of networks for which not all neurons on a given sheet have the same fanin. Thus, the value stored here is used only as a "flag" so that, during execution, it can be determined whether or not two sheets are linked.

\section{CORTPARM(2,KS,NS,KT,NT)}

Maximum strength (magnitude) for activating connections linking (KS,NS) and $(\mathrm{KT}, \mathrm{NT})$. Values for this parameter are required to lie in the range 0.0 to plus 1.0.

\section{CORTPARM(3,KS,NS,KT,NT)}

Maximum strength (magnitude) for inhibiting connections linking (KS,NS) and $(\mathrm{KT}, \mathrm{NT})$. Values for this parameter are required to lie in the range 0.0 to plus 1.0.

\section{CORTPARM(4,KS,NS,KT,NT)}

Learning amplitude for connections linking (KS,NS) and (KT,NT). The value here is used as a multiplier for computed weight alteration of both signs. Values for this parameter are required to lie in the range 0.0 to plus 1.0. At present, no provision is made for training-dependent modification of the learning amplitude.

\section{CORTPARM(5,KS,NS,KT,NT)}

Decanting parameter for connections between (KS,NS) and (KT,NT). Decanting refers (for the purposes of the program!) to the reduction in connection catalyst which is associated with the weight alteration process. In contradistinction to the Connection Catalyst Decrement parameters noted below, the Decanting parameter does not function in a manner dependent upon the magnitude of the associated weight alteration. Instead, with each alteration attempt (whether successful or not) an amount of catalyst determined by this parameter is removed from the associated connection. A value of 0.0 implies that decanting does not 
take place, a value of 1.0 that decanting is total. Intermediate values have the obvious effect. Values outside this range are ignored. It should be noted that, in the present eddy-current realization of the code, the decanting process has been eliminated.

\section{CORTPARM(6,KS,NS,KT,NT)}

Connection Catalyst Decrement value for activating connections linking source neurons on (KS,NS) and target neurons on (KT,NT). This parameter establishes the rate at which weight alteration-dependent catalyst usage occurs for activating connections. Values in the range 0.0 to the maximum connection catalyst limit value (usually 32000 . units) are permitted.

\section{CORTPARM(7,KS,NS,KT,NT)}

Connection Catalyst Decrement value for inhibiting connections linking source neurons on (KS,NS) and target neurons on (KT,NT). This parameter establishes the rate at which weight alteration-dependent catalyst usage occurs for inhibiting connections. Values in the range 0.0 to the maximum connection catalyst limit value (usually 32000. units) are permitted.

\section{CORTPARM(8,KS,NS,KT,NT)}

Weight Alteration Asymmetry parameter for connections linking source neurons on (KS,NS) and target neurons on (KT,NT). Values greater than unity force inhibiting connections to "learn" faster than activating ones and conversely. A value of "1." forces equality.

\section{CORTPARM(9,KS,NS,KT,NT)}

"ALGORITHMIC" Indicator. A non-zero value here implies that the transfer of neural signals, from whatever the source sheet (e.g., some (KSS,NSS)), to (KT,NT) will be, in some code-dependent fashion, mediated algorithmically by the signals appearing on a third sheet, (KS,NS). This mediation will be directed by the spatial and intensity distribution on (KS,NS), a distribution neurally derived from the signals appearing at (KSS,NSS). The effective single "source" for (KT,NT) is taken to be the source (KSS,NSS), the source for (KS,NS) itself, and denoted by a "1" in CORTPARM(10,KSS,NSS,KS,NS). In effect, the distribution on (KS,NS) determines the regions on (KSS,NSS) which will be transferred to a (KT,NT). The value of parameter 9 denotes the position in the transfer sequence from (KSS,NSS) at which (KT,NT) is to accept inputs. As a simple (and previously explored) example, it may be arranged that the 5 "brightest" regions (of size determined by the code in a manner unimportant here) of the retina (at $(1,1))$ are successively "copied" onto the external inputs of sheets $(3,1),(3,2),(3,3)$, $(3,4),(3,5)$. In this example, (KSS,NSS) is $(1,1),(\mathrm{KS} . N S)$ is the mediating sheet (perhaps at $(2,1)$ ), and the Target sheets are successively $(3,1)$ through $(3,5)$. For reasons associated with the problems for which the algorithmic mediation scheme was developed, the method has come to be referred to as "Prosthetic Transfer". Although not supported by present versions of the code, the Prosthetic Transfer scheme proved very useful in connection with earlier attempts to model some of the processes thought to be associated with the lateral geniculate bodies. It should be noted that, if a non-zero value for parameter 9 should be encountered 
during the connection checking process, tests are performed to ensure the presence of the necessary contributing neural sheets.

CORTPARM(10,KS,NS,KT,NT)

Mediating Source Sheet Parameter. $A$ "1" in this position indicates that sheet

(KS,NS) is to serve as the effective source for algorithmically mediated transfer of neural signals to one or more of those sheets for which (KT,NT) is itself a source. The distinctions among the three sheet categories involved in algorithmic transfer may be clarified by noting that, for the example suggested above, there would be an entry of " 1 " in CORTPARM $(10,1,1,2,1)$, and entries of " 1 " in CORTPARM(9,2,1,3,1), "2" in CORTPARM(9,2,1,3,2), "3" in CORTPARM(9,2,1,3,3), "4" in CORTPARM(9,2,1,3,4), and "5" in CORTPARM $(9,2,1,3,5)$.

As suggested earlier, the elements of the CONNPARM array define, in terms of "connection classes", the spatial distribution, initial weight, etc. for groups (or "classes") of connections linking specific sheet pairs. It will be important to bear in mind that a CONNPARM class is applicable to all target sheet neurons within its range of definition (either the entire target sheet or, if defined, a subsheet thereof). Within the limits set by the system parameter NCONNSYS, there may be arbitrarily many connection classes linking two neural sheets. The possibility of a multiplicity of linking connection classes (each involving the associated source and target neurons in a potentially different mode of connectivity) coupled with the nature of the class-defining parameters permit the establishment of a very broad range of initial connection distributions. However, and this point is important, once the initial connectivity is established, reference to class-defining parameters is forbidden (except, of course, as required by the listing utilities). Thus, however distributed, all connections (whatever their class origin) linking a given pair of sheets are processed in the context of the entries in the corresponding CORTPARM entries for the sheet pair.

In the following discussion of the parameters represented by the CONNPARM array, the variables KS, NS, KT, and NT have the meanings already defined. The variable $L$ denotes connection class. Because more than half of the connection-defining parameters can be represented in terms of type INTEGER* 2 values (indeed, in most cases by INTEGER*1 values), the single array earlier called CONNPARM is in fact divided into two components, an integer part and a real part. These will frequently be referred to collectively as CONNPARM (indeed, the data-file elements for a given class are contiguous) but are employed in their separate forms, the integer part referred to as ICONNPARM and the real part by

CONNPARM. For any $\mathrm{L},(\mathrm{KS}, \mathrm{NS})$ and $(\mathrm{KT}, \mathrm{NT})$, the following definitions obtain:

\section{ICONNPARM(1,L,KS,NS,KT,NT)}

Fanin for neurons on (KT,NT) with respect to source neurons on (KS,NS) for connection class $\mathrm{L}$. The value here (as distinct from the similarly named entry in CORTPARM) is supplied by the user during system file construction and represents, for class $\mathrm{L}$, the number of connect ons linking a source region on level/sheet (KS,NS) to a typical neuron on (KT,NT). The characteristics of the source region are defined below. 
The next two entries are the so-called "Mapping Parameters". These determine the manner in which a region on the source sheet is mapped by (i.e., defined by the coordinates of) a target neuron. If a MAP parameter equals 1, scaled mapping of target neuron positions onto source sheets is performed in the indicated coordinate. Thus, for the indicated coordinate, the center of the source region is determined by linear proportion as a function of the target neuron coordinate and the relative sheet sizes. If a MAP parameter is 0 , the mapping point is taken as the source sheet midpoint (or the midpoint of the source subsheet, if one is defined) in the indicated coordinate. Note that the range, wavelength, and parity parameters remain functional.

ICONNPARM(2,L,KS,NS,KT,NT)

IMAP parameter for connections from (KS,NS) to (KT,NT) for connectivity class L.

ICONNPARM(3,L,KS,NS,KT,NT)

JMAP parameter for connections from (KS,NS) to (KT,NT) for connectivity class $\mathrm{L}$.

The next two entries, IRANGE and JRANGE, define the region of neural sheet (KS,NS) from which a typical neuron on (KT,NT) can accept inputs for connectivity class $L$. Note that, in earlier versions of the program, IRANGE and JRANGE were interpreted as decimal fractions (in the range $.0->1$.) of the corresponding dimensions of the relevant cortical sheet and that these fractions, when converted into "connectivity ranges", referred respectively' to the half-depth and half-width (or, more accurately put, to one less than the half-dimension) of the source region.

In the current version of the code, IRANGE and JRANGE are stored and utilized as explicit (absolute) coordinate distances from the mapping position determined from the MAP parameter values described above.

A point to note carefully is that, during the connection generation process, the source region center is taken as the current mapping point, generally referred to in the code by the variable pair (IMAP,JMAP). For the purpose of computing available cortical source space as preparation for network construction, IMAP and JMAP are taken equal to 0 . This point is unrelated to (and should not be permitted to confuse) the issue of cortical sheet origins (which are always at position $(I=1, J=1)$ ).

ICONNPARM(4,L,KS,NS,KT,NT)

IRANGE parameter for connections from (KS,NS) to (KT,NT) for connectivity class $\mathrm{L}$.

ICONNPARM(5,L,KS,NS,KT,NT)

JRANGE parameter for connections from (KS,NS) to (KT,NT) for connectivity class $\mathrm{L}$.

The "periodicity" parameters for the two coordinate axes confer on the system constructor the capability for generating wiring distributions in which, for any defined source region, only every Nth connection along the specified axis is made where " $N$ " is the periodicity value. 
ICONNPARM(6,L,KS,NS,KT,NT)

Periodicity in "I" for connections between (KS,NS) and (KT,NT) for connectivity class L.

ICONNPARM(7,L,KS,NS,KT,NT)

Periodicity in "J" for connections between (KS,NS) and (KT,NT) for connectivity class L.

The following "parity" entries define in the corresponding Source sheet coordinates the offset of the source neuron region center from the corresponding coordinate as it has been specified (through the mapping parameters) by the position of the target neuron. Note that, in earlier versions of the program, it was required that the parity be either even (0) or odd (1). In the present code, any integer value is permitted.

ICONNPARM(8,L,KS,NS,KT,NT)

"I" parity for connections between (KS,NS) and (KT,NT) for connectivity class L.

ICONNPARM(9,L,KS,NS,KT,NT).

"J" parity for connections between (KS,NS) and (KT,NT) for connectivity class L.

ICONNPARM(10,L,KS,NS,KT,NT).

(I/J) Edge-Factor. If the value of this parameter is 0 , target-to-source mapping is performed without regard to the values of the "range" parameters. If the value is 1 , the mapping constants are computed as though the source sheet were one range parameter smaller in each dimension (Note that there are not separate I and $\mathrm{J}$ edge-factor parameters). Considered use of this parameter results (among others) in a convenient method for producing accurate "condensing" wirings.

ICONNPARM(11,L,KS,NS,KT,NT)

DUMMY (Once used for "N" $" T " / " O "$ terminal connection indication).

ICONNPARM(12,L,KS,NS,KT,NT)

"EXT_I0"/"POIN" Flag. A value of 0 for this parameter indicates that connections of class L linking (KS,NS) and (KT,NT) are attached to the "External Inter-Neural" (or I0 as it often appears in the code) input terminal of the target neuron. A value of 1 indicates attachment to the normal, or post-synaptic input (POIN) terminal. For a discussion of the distinction between these two input types, see the main body of the text.

\section{ICONNPARM(13,L,KS,NS,KT,NT)}

Self-Linking factor for connections linking source neurons on (KS,NS) and target neurons on (KT,NT) for connectivity class $\mathrm{L}$ ( 1 if self-linking is permitted, 0 otherwise). The value of this parameter must be 1 if, for example, any neurons on the associated sheet are to be given "feedback" from their own outputs.

The remaining eight ICONNPARM entries permit the definition of distinct subsheets on both source (KS,NS) and target (KT,NT) sheets linked by connections of class L. In 
effect, these parameters make possible the division of one larger sheet (whether source or target) into independently functioning smaller sheets.

ICONNPARM(14,L,KS,NS,KT,NT)

The extent in the I coordinate (depth) of a subsheet of a sheet (KS,NS) serving as the source for (KT,NT).

ICONNPARM(15,L,KS,NS,KT,NT)

The extent in the $\mathrm{J}$ coordinate (width) of a subsheet of a sheet (KS,NS) serving as the source for (KT,NT).

ICONNPARM(16,L,KS,NS,KT,NT)

The I coordinate center of the subsheet denoted by

ICONNPARM(14,L,KS,NS,KT,NT) and ICONNPARM(15,L,KS,NS,KT,NT).

ICONNPARM(17,L,KS,NS,KT,NT)

The J coordinate center of the subsheet denoted by

ICONNPARM(14,L,KS,NS,KT,NT) and ICONNPARM(15,L,KS,NS,KT,NT).

ICONNPARM(18,L,KS,NS,KT,NT)

The extent in the I coordinate (depth) of a subsheet of target sheet (KT,NT) served by the source (KS,NS).

ICONNPARM(19,L,KS,NS,KT,NT)

The extent in the $J$ coordinate (width) of a subsheet of target sheet (KT,NT) served by the source (KS,NS).

ICONNPARM(20,L,KS,NS,KT,NT)

The I coordinate center of the subsheet denoted by

ICONNPARM(18,L,KS,NS,KT,NT) and ICONNPARM(19,L,KS,NS,KT,NT).

ICONNPARM(21,L,KS,NS,KT,NT)

The J coordinate center of the subsheet denoted by

ICONNPARM(18,L,KS,NS,KT,NT) and ICONNPARM(19,L,KS,NS,KT,NT).

The remaining seven connection-defining parameters are more conveniently expressed as decimal values. These are stored in the array, CONNPARM, whose elements are described below. The already established conventions apply.

CONNPARM(1,L,KS,NS,KT,NT)

Initial weight value (expressed as a signed fraction of the maximum weight linking (KS,NS) and (KT,NT)) for connectivity class L. Thus, during network

construction the value here is multiplied by the value of

CORTPARM(2,KS,NS,KT,NT) if the sign is positive and by

CORTPARM(3,KS,NS,KT,NT) otherwise.

CONNPARM(2,L,KS,NS,KT,NT)

Local Radial Weighting exponent for connections linking source neurons on (KS,NS) and target neurons on (KT,NT) for connectivity class $\mathrm{L}$. The value appearing here determines the rate at which connection strengths increase (or 
decrease) as a function of distance from mapping point to connection source position.

CONNPARM(3,L,KS,NS,KT,NT)

Global Radial Weighting factor for connections linking source neurons on (KS,NS) and target neurons on (KT,NT) for connectivity class $\mathrm{L}$. For the present, a value of -1 indicates that connections originating from a point closer to an implied sheet center than the target neuron are not constructed. A value of +1 indicates that connections originating from a point more distant from an implied sheet center than the target neuron, or equidistant from it, are not constructed.

\section{CONNPARM(4,L,KS,NS,KT,NT)}

Global Radial Weighting exponent for connections linking source neurons on (KS,NS) and target neurons on (KT,NT) for connectivity class L. The entry here differs from the Local Radial Weighting exponent in that it is distance from source sheet center, rather than distance from mapping position, which is determinative.

\section{CONNPARM(5,L,KS,NS,KT,NT)}

Local Azimuthal Weighting exponent for connections linking source neurons on (KS,NS) and target neurons on (KT,NT) for connectivity class $\mathrm{L}$. This parameter has meaning only for sheets with an implied central origin and refers to the angle between the target origin and target source directions.

\section{CONNPARM(6,L,KS,NS,KT,NT)}

Global Azimuthal Weighting parameter for connections linking source neurons on (KS,NS) and target neurons on (KT,NT) for connectivity class L. This parameter has meaning only for sheets with an implied central origin and refers to the angle between the target-origin direction and the " $\mathrm{Z}$ ", or vertical, direction (an assumed perpendicular joining source and target sheets).

\section{CONNPARM(7,L,KS,NS,KT,NT)}

Initial Connection Catalyst for connections linking source neurons on (KS,NS) and target neurons on (KT,NT) for connectivity class L (expressed as a fraction of the system-wide parameter KATSYS0).

All the parameters described so far contribute in one way or another to the description of those neuron and connection distributions characteristic of some neural network and are (with few exceptions), as previously noted, supplied by the user in the form of a system parameter file. The remaining defining parameter type specifies the characteristics, not of the neural and connection distributions, but of the response of neurons to input signals. Values for parameters of this type are stored in the array SIGPARM which, like LEVELPARM, is defined in terms of one set (here specifically the target set) of neural sheet coordinates. For reference purposes, we display as Figure 4 the general form of the neuron response function which the SIGPARM values (more correctly, the first four thereof) define. Although approximately Sigmoid in shape, the precise form of the region between the onset and saturation input values is a combination of a Sigmoid and a vertically displaced half-cosine. For clarity, we anticipate subsequent material and note that the Sigmoid function for each defined 
(KT,NT) is stored in a "lookup table", or array, of the form SIGMOID(KT,NT,INDEX) so that, where required, unique response functions can be associated with individual neural sheets. As noted below, the variable INDEX is derived from the neuron input and associates that input with the proper neuron output value.

ORNL-DWG 92-11935

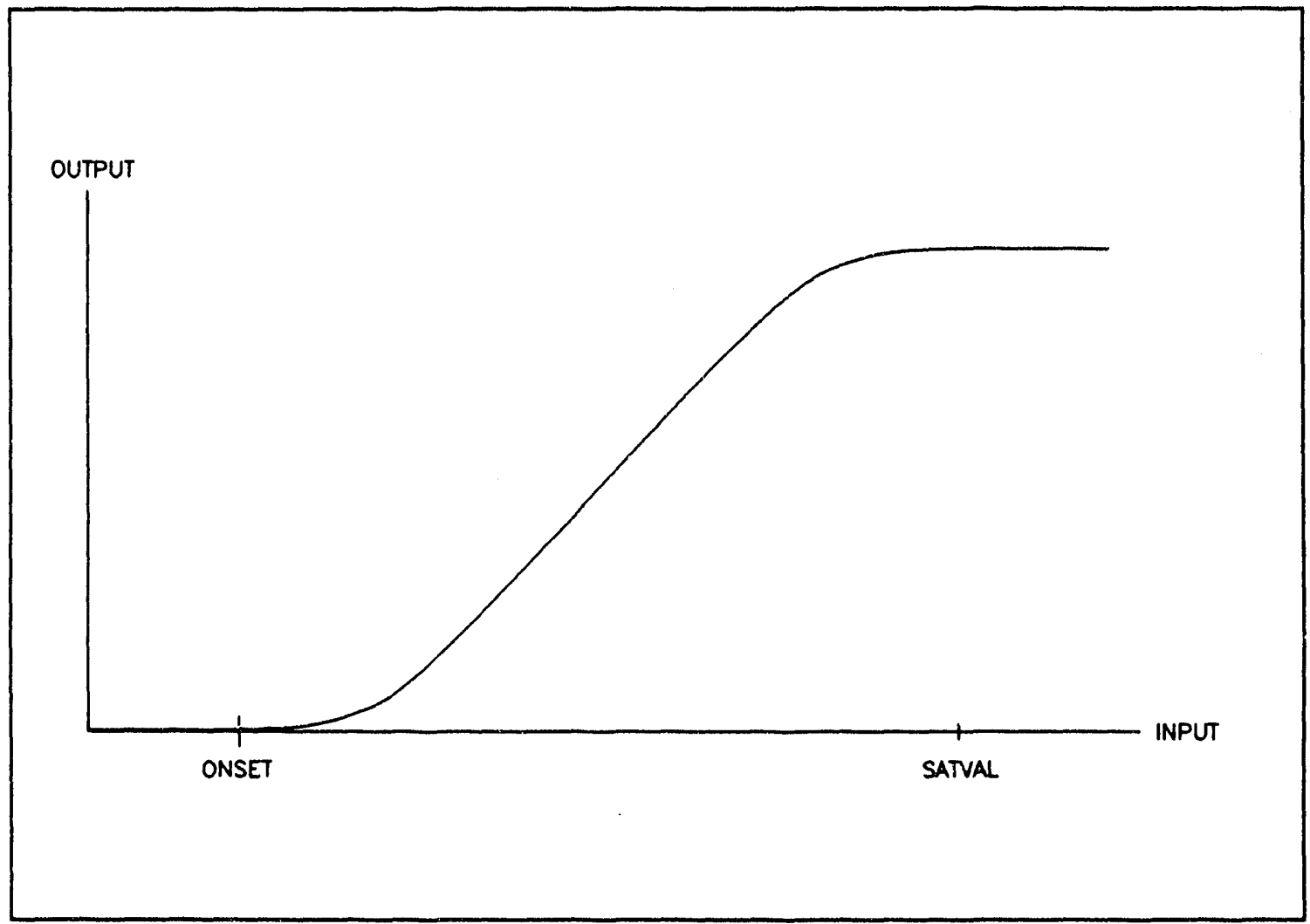

Fig. 4. Sigmoid response function.

For any neuron-bearing region defined by the pair $(\mathrm{KT}, \mathrm{NT})$, the following definitions obtain:

\section{SIGPARM(1,KT,NT)}

Sigmoid index limit for the Sigmoid function associated with neurons on sheet NT of level KT. In earlier versions of the code, the value of this parameter determined the resolution to which a neural input could be defined. In the interest of maximizing execution speed, this scaling factor is no longer employed and resolution is limited to the number of integer steps between the onset and saturation input values.

Note that the following two parameters are stored in files as decimal fractions and are interpreted by the program (and stored internally) in terms of the output limit for neurons of the corresponding level and sheet stored in LEVELPARM(2,KT,NT). 
SIGPARM(2,KT,NT)

Onset input value (ONSET) for the Sigmoid function associated with neurons on (KT,NT). The value of this parameter determines the minimum input required for producing non-zero output.

SIGPARM(3,KT,NT)

Maximum unsaturated input (SATVAL) for the Sigmoid function associated with neurons on (KT,NT). The value here establishes the input which yields the maximum output.

\section{$\operatorname{SIGPARM}(4, \mathrm{KT}, \mathrm{NT})$}

Minimum Neuron Output Limit factor. This parameter is stored as that fraction of the Sigmoid Onset Value which, when added to ONSET establishes the input value from which the neuron lower limit of activity (NEURONONLIM) is determined. During system preparation the computed value of NEURONONLIM replaces the data set parameter according the prescription:

$$
\text { ONSETMAX =ONSET+SIGPARM(4,KT,NT)*(SATVAL-ONSET) }
$$

NEURONONLIM itself is given by:

$$
\begin{aligned}
& \text { NEURONONLIM }=\text { SIGMOID(KT,NT,INT(SIGFACT +SIGPARM(6,KT,NT)* } \\
&\text { ONSETMAX) })
\end{aligned}
$$

Recent versions of the code have made no use of this parameter.

SIGPARM(5,KT,NT)

Post-Synaptic/10 Input Error Factor. The value of this parameter is employed in two ways. For 10-driven sheets, it determines the disparity which will be tolerated between signals deriving from "trained" sources (POIN inputs) and those representing "expected" response (I0 inputs). Although these latter may, by suitable arrangements of interneural wiring, be injected at any point in a network, they are most commonly applied at the "top" of a "column" or "pyramid". For details, see the main body of the text. Observe that this parameter is used multiplicatively in the context of the neuron input range defined by the difference (SATVAL - ONSET). The necessary conversions are performed during the SIGMOID generation process.

For neural sheets which are not 10 -driven, the value of this parameter establishes the magnitude of the dither operation by which the neurons on such sheets determine whether or not training is required.

\section{SIGPARM(6,KT,NT)}

Sigmoid Index conversion factor for the Sigmoid function associated with neurons on (KT,NT). When employed (it is not in present systems) it appears in the transformation relation which associates post-synaptic signal values in the range (ONSET-->SATVAL) with indices into the SIGMOID array. Note that this entry, like the one above, is computed during preparation of the system. The form of the transformation is: 
INDEX $=1+B^{*}($ POST-SYNAPTIC_INPUT - ONSET $)$

in which "B" is the value stored in $\operatorname{SIGPARM}(6,$,$) .$

SIGPARM(7,KT,NT)

Sigmoid Input-> Output conversion constant. For those program versions in which it appears, the combination of terms noted below appears with such frequency as to warrant its precomputation and storage.

\section{SIGFACT $=1 .-$ SIGPARM $(6, \mathrm{KT}, \mathrm{NT}){ }^{*}$ ONSET}

With the descriptions of the SIGPARM array elements we complete our survey of the systemdefining arrays over which the system constructor has any direct control. We devote the remainder of this section to a discussion of those arrays which are filled either during the network generation process or during program execution. Although it is principally the set of connections and the rules by which they are altered that confer any degree of individuality on neural network methodology, it will prove more convenient to begin our discussions not with the connectivity-related arrays, but with the array NEURON whose elements define the states of the neurons comprising a LILARTI network.

A point of clarification may prove helpful before we begin. The attentive reader will recall that we have so far defined neurons in terms of their four coordinates in "network space". Of these coordinates, two, $\mathrm{K}$ and $\mathrm{N}$, have specified the position of the neural sheet upon which some specified neuron resides and two, I and $\mathrm{J}$, have denoted the position on the sheet identified by $\mathrm{K}$ and $\mathrm{N}$. Although this representation is very convenient for the user, it can be extremely inefficient in terms of storage space and very limiting of processing speed for sparse networks of the LILARTI variety. Both of these liabilities can be circumvented neatly by the adoption of a form of pointer-directed storage scheme. The details of the method will emerge as we describe the entries in the NEURON array and the neuron pointer array NPOINT. For the moment we need only remark that in order to access the parameters for a specified neuron (e.g., the neuron at position $(\mathrm{K}, \mathrm{N}, \mathrm{I}, \mathrm{J})$ a pointer value (NP) is derived from a starting position value stored in [NPOINT $(1, K, N)]$, the sheet width stored in $[\operatorname{LEVELPARM}(4, \mathrm{~K}, N)]$, and the values of $I$ and $J$. Indeed, except for the input/output functions intended for user edification, neurons are referred to (and accessed only by) pointer position. For any pointer value NP, the following definitions apply:

NEURON(1,NP)

External Sensor Input to neuron NP

NEURON(2,NP)

External Inter-Neural Input to neuron NP

NEURON(3,NP)

Post-Synaptic Input for neuron NP from sources other than External Sensor Inputs and External Inter-Neural Inputs

NEURON(4,NP)

Current "N" Output of neuron NP 
NEURON(5,NP)

Old "N" Output for neuron NP

NEURON(6,NP)

Current "DN" Output of neuron NP

NEURON(7,NP)

Old "DN" Output for neuron NP

NEURON $(8, N P)$

Received_Target_Error (R_T_E) for neuron NP

NEURON $(9, N P)$

Differential RTE for neuron at $(\mathrm{I}, \mathrm{J})$ on sheet $\mathrm{N}$ of cortical level $\mathrm{K}$

NEURON(10,NP)

Dither_Done_Flag for neuron NP

NEURON(11,NP)

Source_Training_Flag (S_T_F) for neuron NP

NEURON(12,NP)

Temporary storage for computed "N" output for neuron NP during the

PREPROCESSING, PROCESSING, and RELAX operations

\section{NEURON(13,NP)}

Save "N" Output for neuron NP "RELAX" processing

The next two entries are DUMMY parameters which serve a variety of purposes during several phases of processing. Observe that some versions of the code may make use of neither, one, or both storage locations.

\section{NEURON(14,NP)}

\section{NEURON(15,NP)}

The next parameters are required to support the generalized connection distributions which can be generated by the present version of the code. KSNSISJS is the connectivity array and stores in contiguous chunks the specifications for those connections which serve as inputs to neurons denoted by values of the pointer NP. Thus, NEURON(16,NP) and NEURON $(17, N P)$ are, in effect, pointers to locations in KSNSISJS. Note that any one of the following three is redundant and that the code could be written to eliminate it:

\section{NEURON(16,NP)}

Starting Location in [KSNSISJS] for input connections to neuron NP

NEURON(17,NP).

Ending Location in [KSNSISJS] for input connections to neuron NP 
NEURON(18,NP).

Total number of input connections to neuron NP.

The NPOINT array facilitates the condensing of neural storage for sparse systems. Its two elements identify the region in the NEURON array in which neuron parameters for a specified level $\mathrm{K}$ and sheet $\mathrm{N}$ are stored.

NPOINT(1,K,N)

Starting position in the array [NEURON] for neurons on $(\mathrm{K}, \mathrm{N})$.

NPOINT(2,K,N)

Ending position in the array [NEURON] for neurons on $(\mathrm{K}, \mathrm{N})$.

Connections in the present LILARTI are defined by the values of eight parameters. Of these, five can be stored as INTEGER* 1 constants in the array KSNSISJS. The remainder, because of their greater range, must be stored as INTEGER*2 constants and reside in the NPSOURCE array. The variable NCONN is, as already noted, effectively a pointer and, for a given target neuron NP, takes on values between NEURON(15,NP) and NEURON(16,NP) to retrieve the range of connections which serve NP as sources.

Note that, although the first four of the following KSNSISJS entries serve primarily to support user input-output functions, they are used for some of the logical decisions required for present LILARTI processing and could not readily be eliminated:

KSNSISJS(1,NCONN)

Source Neuron 'K' Coordinate (KS).

KSNSISJS(2,NCONN)

Source Neuron 'N' Coordinate (NS).

KSNSISJS(3,NCONN)

Source Neuron 'I' Coordinate (IS).

KSNSISJS(4,NCONN)

Source Neuron 'J' Coordinate (JS).

KSNSISJS(5,NCONN)

Target Neuron 'EXT_I0'/POIN' Flag. A value of 0 indicates that the source signal is to be applied to the External Inter-Neural Input terminal of the target neuron. A value of 1 implies use of the normal, or post-synaptic input.

The remaining connectivity parameters are stored in the INTEGER*2 array NPSOURCE as noted below.

NPSOURCE(1,NCONN)

Weight of link from a source neuron at (KS,NS,IS,JS) (indirectly pointed to by the value of NCONN) to the target neuron for which NCONN is a connection pointer. Weights span the range -1 . to 1 . inclusive but are represented by integers 
in the range $-16 \mathrm{~K}$ to $+16 \mathrm{~K}$. Almost all of the computations involving weights make use of the integer representation in the interest of processing efficiency.

NPSOURCE(2,NCONN)

Remaining connection catalyst for connection alterations involving link from (KS,NS,IS,JS) to the target neuron for which NCONN is a connection pointer. Catalyst values span the range 0 to the value represented by the system variable KATSYS0 (usually 32000).

NPSOURCE(3,NCONN)

Pointer to Source Neuron (almost always referred to by the variable NPS) for connection alterations involving link from (KS,NS,IS,JS) to the target neuron for which NCONN is a connection pointer. The value of NPS permits direct access to the parameters for the neuron at the source end of the connection identified by NCONN.

The SIGMOID array represents for each neural sheet the response function which relates neuron input to neuron output. Depending upon the version of the code the index "I" which appears as an argument in SIGMOID() is either equal to the presently obtaining input or generated from the input according to the prescription:

$$
\mathrm{I}=\operatorname{INT}(\operatorname{SIGPARM}(7, \mathrm{~K}, \mathrm{~N})+\operatorname{SIGPARM}(6, \mathrm{~K}, \mathrm{~N}) * \operatorname{INPUT}) \text {. }
$$

\section{$\operatorname{SIGMOID}(K, N, I)$}

Functional component of neuron response for input index I for neurons on sheet $\mathrm{N}$ of level $\mathrm{K}$.

Related to the Sigmoid array is its inverse SIGMOIDINV which finds application during the computation of post-synaptic input error values.

\section{SIGMOIDINV(K,N,IOUTPUT)}

Inverse of neuron response function. For any $(\mathrm{K}, \mathrm{N})$, the stored value for an index, IOUTPUT, is the input which would produce it. The numerical value of IOUTPUT is equal to the neural output value for which a corresponding input is desired and must lie between 0 and LEVELPARM(2,K,N).

We note next the several "bookkeeping" arrays which are generated during system setup and/or execution. These arrays serve in one manner or another either to reduce execution time (by codifying frequently required combinations of parameters or "conditions") or to collect data potentially useful to a user attempting to optimize system behaviour.

\section{KNSDEFINED(K,N)}

Defined Source List. The elements of this array denote (by a 1 or 0 in location $(\mathrm{K}, \mathrm{N}))$ those neural sheets from which input connections originate.

\section{KNTDEFINED(K,N)}

Defined Target List. The elements of this array denote (by a 1 or 0 in location $(\mathrm{K}, \mathrm{N}))$ those neural sheets on which input connections terminate. 
In connection with the above two arrays, it should be noted that, although most neural sheets in a LILARTI system act as both source and target, this is not required. The retina, for instance, is usually connected only as a source, although it is certainly possible to endow it with any desired connectivity characteristics (e.g., with some "nearest-neighbour" lateral inhibition). The "keyboard cortex" regions, on the other hand, generally serve only as targets although it is occasionally prudent to provide for both a little self-activation and a little mutual inhibition.

\section{KNLEARN(K,N)}

Trainable Neural Sheet List. The elements of this array denote (by a 1 or 0 in location $(K, N)$ ) those neural sheets on which trainable input connections (i.e., those bearing non-zero initial connection catalyst) terminate.

\section{KNRANCONN(KS,NS,KT,NT)}

Random Connection List. The elements of this array denote (by a 1 or 0 in location $(\mathrm{K}, \mathrm{N})$ ) those neural sheet pairs between which the random connection generation process has been invoked. Note that (KS,NS) and (KT,NT) may define the same neural sheet.

\section{$\mathrm{KNCOMP(3)}$}

Keyboard Complementary Sheet Definitions. The first entry signifies the level (K) at which the complementary sheets reside, the second the sheet number $(N)$ of the first of these sheets, and the third, the number of contiguous sheets making up the region. If all is well, this latter value will equal the number of image defining parameters.

\section{KNEXTI0(1/2,K,N)}

External Sensor Input Flag/Inter-Neural External Input Flag for Level/Sheet $(\mathrm{K}, \mathrm{N})$. The entries here are set during processing as it is determined that one or the other (or both) of the noted input signal types is/are present at the input for at least one neuron on $(\mathrm{K}, \mathrm{N})$. Note that the entries here will vary during processing.

\section{$\operatorname{KNPHASE}(1 / 2, \mathrm{~K}, \mathrm{~N})$}

Bookkeeping array for Phase State of Level/Sheet $(K, N)$. The first entry denotes the number of neurons on neural sheet $(K, N)$ which have not yet undergone the "Dither" operation. The second denotes the number which have not yet undergone source-connection training.

\section{$\mathrm{KNCOPY}(\mathrm{K}, \mathrm{N})$}

The elements of this array identify those neural sheets which must be copied into temporary storage for the dither processing operations.

The next noted array stores the results of four neural sheet-wide assessments of system activity:

\section{$\operatorname{ACTIVITY}(1, \mathrm{~K}, \mathrm{~N})$}

Total Activity for sheet (K,N) (sum of all neuron "N" outputs). 
ACTIVITY $(2, \mathrm{~K}, \mathrm{~N})$

Specific Activity for sheet (K,N) (Total Sheet Activity/Number of Active

Neurons).

ACTIVITY $(3, \mathrm{~K}, \mathrm{~N})$

Current Stability value for neural sheet $(\mathrm{K}, \mathrm{N})$.

ACTIVITY $(4, \mathrm{~K}, \mathrm{~N})$

Last Stability value for neural sheet $(K, N)$.

The STATKN array, whose elements are noted below, serves to collect various measures of system performance during the training process. Because the stored information is principally useful during the initial phases of system development, provision is made for defeating the statistics collection when the resulting data are not required.

STATKN $(1,1, \mathrm{KS}, \mathrm{NS}, \mathrm{KT}, \mathrm{NT})$

Single Iteration Number of Activating Alterations. Stored here is the total number of connection strength alterations involving activating connections linking neural sheets (KS,NS) and (KT,NT) occurring during a single iteration. Needless to say, the value of this parameter is re-set to 0 before each iteration.

\section{STATKN(2,1,KS,NS,KT,NT)}

Single Iteration Sum of Activating Alterations. Stored here is the sum of connection strength alterations involving activating connections linking neural sheets (KS,NS) and (KT,NT) occurring during a single iteration. The value of this parameter is also re-set to 0 before each iteration.

The following two storage locations serve inhibiting connections in the just the manner noted above for activating connections.

STATKN(3,1,KS,NS,KT,NT)

Single Iteration Number of Inhibiting Aiterations.

\section{STATKN $(4,1, \mathrm{KS}, \mathrm{NS}, \mathrm{KT}, \mathrm{NT})$}

Single Iteration Sum of Inhibiting Alterations.

The following four storage locations serve the catalyst decanting process in a manner analogous to the just noted scheme serving the weight alteration process. Recall that the current version of the code does not support the decanting.

STATKN $(5,1, \mathrm{KS}, \mathrm{NS}, \mathrm{KT}, \mathrm{NT})$

Single Iteration Number of Activating Decantations.

STATKN(6,1,KS,NS,KT,NT)

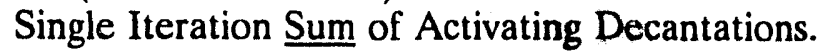

STATKN(7,1,KS,NS,KT,NT)

Single Iteration Number of Inhibiting Decantations. 


\section{STATKN $(8,1, \mathrm{KS}, \mathrm{NS}, \mathrm{KT}, \mathrm{NT})$}

Single Iteration Sum of Inhibiting Decantations.

The following eight storage locations function as accumulators for the eight single iteration parameters just noted. The accumulation process continues without resetting during any of the system training modes. Restarting the training process results in resetting all STATKN entries.

STATKN $(1,2, \mathrm{KS}, \mathrm{NS}, \mathrm{KT}, \mathrm{NT})$

Accumulated Number of Activating Alterations.

STATKN(2,2,KS,NS,KT,NT)

Accumulated Sum of Activating Alterations.

STATKN(3,2,KS,NS,KT,NT)

Accumulated Number of Inhibiting Alterations.

STATKN(4,2,KS,NS,KT,NT)

Accumulated Sum of Inhibiting Alterations.

STATKN(5,2,KS,NS,KT,NT)

Accumulated Number of Activating Decantations.

STATKN(6,2,KS,NS,KT,NT)

Accumulated Sum of Activating Decantations.

STATKN(7,2,KS,NS,KT,NT)

Accumulated Number of Inhibiting Decantations.

\section{STATKN(8,2,KS,NS,KT,NT)}

Accumulated Sum of Inhibiting Decantations.

The following eight storage locations serve to save the largest values of, respectively, each of the single iteration entries noted above. Each "maximum value" location is updated at the end of each training iteration if its currently stored value is exceeded by the corresponding single iteration value just acquired.

STATKN(1,3,KS,NS,KT,N')

Maximum Single Ittration Number of Activating Alterations.

STATKN(2,3,KS,NS,KT,NT)

Maximum Single Iteration Sum of Activating Alterations.

STATKN(3,3,KS,NS,KT,NT)

Maximum Single Iteration Number of Inhibiting Alterations.

\section{S' $\Gamma$ ATKN (4,3,KS,NS,KT,NT)}

Maximum Single Iteration Sum of Inhibiting Alterations. 
$\operatorname{STATKN}(5,3, \mathrm{KS}, \mathrm{NS}, \mathrm{KT}, \mathrm{NT})$

Maximum Single Iteration Number of Activating Decantations.

STATKN(6,3,KS,NS,KT,NT)

Maximum Single Iteration Sum of Activating Decantations.

$\operatorname{STATKN}(7,3, \mathrm{KS}, \mathrm{NS}, \mathrm{KT}, \mathrm{NT})$

Maximum Single Iteration Number of Inhibiting Decantations.

$\operatorname{STATKN}(8,3, \mathrm{KS}, \mathrm{NS}, \mathrm{KT}, \mathrm{NT})$

Maximum Single Iteration Sum of Inhibiting Decantations.

The KNSOURCE array, whose elements are described below, serves to collect, for a specific target neuron, inputs and source neuron counts on a per/neural sheet basis. Now used only in connection with various user utilities, the array was employed during training in several earlier versions of the LILAR'TI system, versions in which weight alterations for connections linking several neural source sheets to the current target were arranged to depend on the overall distribution of source signals. The KNSOURCE elements are defined below.

\section{KNSOURCE(1,KS,NS)}

Total Input to Current Target Neuron from Source Sheet (KS,NS). Stored here is the sum of all inputs, without regard to type, having their origin in source neurons on neural sheet (KS,NS).

\section{KNSOURCE(2,KS,NS)}

External Inter-Neural Input to Current Target Neuron from Source Sheet (KS,NS). Stored here is the sum of all inputs of the 10 type having their origin in source neurons on neural sheet (KS,NS).

\section{KNSOURCE(3,KS,NS)}

Post-Synaptic Input to Current iarget Neuron from Source Sheet (KS,NS) (excluding contributions from self-activation). Stored here is the sum of all inputs connected to the target "POIN" (post-synaptic) input terminal and having their origin in source neurons on neural sheet (KS,NS).

\section{KNSOURCE(4,KS,NS)}

Catalyst-Bearing, Activating Input to Current Target Neuron from Source Sheet (KS,NS) (Pre-Synaptic). Stored here is the pre-synaptic sum of all activating inputs linked by non-zero catalyst-bearing connections to the target "POIN" (postsynaptic) input terminal and having their origin in source neurons on neural sheet (KS,NS).

\section{KNSOURCE(5,KS,NS)}

Catalyst-Bearing, Inhibiting Input to Current Target Neuron from Source Sheet (KS,NS) (Pre-Synaptic). Stored here is the pre-synaptic sum of all inhibiting inputs linked by non-zero catalyst-bearing connections to the target "POIN" (postsynaptic) input terminal and having their origin in source neurons on neural sheet (KS,NS). 
KNSOURCE(6,KS,NS)

Catalyst-Bearing, Activating Input to Current Target Neuron from Source Sheet (KS,NS) (Post-Synaptic). Stored here is the post-synaptic sum of all activating inputs linked by non-zero catalyst-bearing connections to the target "POIN" (postsynaptic) input terminal and having their origin in source neurons on neural sheet (KS,NS).

\section{KNSOURCE(7,KS,NS)}

Catalyst-Bearing, Inhibiting Input to Current Target Neuron from Source Sheet (KS,NS) (Post-Synaptic). Stored here is the post-synaptic sum of all inhibiting inputs linked by non-zero catalyst-bearing connections to the target "POIN" (postsynaptic) input terminal and having their origin in source neurons on neural sheet (KS,NS).

\section{KNSOURCE(8,KS,NS)}

Counter for Positively Connected "ON" Neurons on Source Sheet (KS,NS). Stored here is the number of neurons on neural sheet (KS,NS) whose output exceeds the neuron "ON" limit for (KS,NS) (usually referred to in the code by the variable NEURONONLIM) and which are connected to the current target "POIN" terminal by at least one activating connection. Note that, when not otherwise defined, NEURONONLIM is zero.

\section{KNSOURCE(9,KS,NS)}

Counter for Negatively Connected "ON" Neurons on Source Sheet (KS,NS). Stored here is the number of neurons on neural sheet (KS,NS) whose output exceeds the neuron "ON" limit for (KS,NS) (usually referred to in the code by the variable NEURONONLIM) and which are connected to the current target "POIN" terminal by at least one inhibiting connection. Note that, when not otherwise defined, NEURONONLIM is zero.

The final arrays we need note are three which serve in the image generation and image perturbation processes. The first, TIMAGEPARM, stores four sets of parameters (three values for each) which define the 81 test images in terms of which much system testing is performed. The test images (which are centered at $X=I R E T / 2, Y=J R E T / 2$ ) are taken to be of the form noted below. The images generated by the indicated expression are intended to mimic in a crude way the gross characteristics of the eddy-current patterns whose analysis has formed the focus of much LILARTI work.

$$
R=\operatorname{SQRT}\left(\operatorname{ABS}\left(\operatorname{SIN}\left(B^{*} T\right)\right)\right)-D^{*} A B S(\operatorname{SIN}(T))^{*} \operatorname{COS}(T / 2 .-C)^{* *} 4
$$

The angle " $\mathrm{T}$ " spans the range 0 to two $\mathrm{Pi}$. From $\mathrm{R}$ are derived the image-defining pixel locations according to the following prescription:

$$
\begin{aligned}
& X 0=A B S(R)^{*} \operatorname{COS}(T) \\
& Y 0=A B S(R)^{*} \operatorname{SIN}(T) \\
& X=A P 1+B P 1 *\left(X 0^{*} \operatorname{COS}(A)+Y 0^{*} \operatorname{SIN}(A)\right) \\
& Y=C P 1+D P 1 *\left(Y 0^{*} \operatorname{COS}(A)-X 0^{*} \operatorname{SIN}(A)\right) .
\end{aligned}
$$


The constants AP1, BP1, CP1, and DP1 are computed during system preparation and lead to a spatial normalization such that the extreme values of the retinal coordinates $(0, \mathrm{IRET} ; 0, \mathrm{JRET})$ are occupied for some set of at most four test images.

The parameters A, B, C, and D are stored in the array TIMAGEPARM in the order noted below. The indices $11->14$ independently take on values from 1 to 3 and thereby retrieve one of the test image data sets per index combination.

TIMAGEPARM $(\mathrm{I} 1,1)==\mathrm{A}$ values

TIMAGEPARM $(12,2)==\mathrm{B}$ values

TIMAGEPARM $(13,3)==C$ values

TIMAGEPARM $(I 4,4)==\mathrm{D}$ values

The array TEMPIMAGE provides temporary storage for pointers to image-defining parameters in the array TIMAGEPARM for the image presently under evaluation. The entries are as noted below:

TEMPIMAGE $(1)==$ Image Number

TEMPIMAGE $(2)==$ Pointer to A storage location

TEMPIMAGE $(3)==$ Pointer to $B$ storage location

TEMPIMAGE $(4)==$ Pointer to $C$ storage location

TEMPIMAGE $(5)==$ Pointer to $D$ storage location

In order to facilitate the study of system response to various forms of image distortion, provision is made for the introduction of combinations of six image perturbation types. These are noted below:

1. Rotation

2. Translation

3. Scale Alteration

4. Random Thinning

5. Addition of Amplitude Noise

6. Addition of Position Noise

Bookkeeping for the perturbation process is provided by the array TEMPERTURB. Provision is made (at present) for applying up to 10 (the current program value of NLABELPARM) successive perturbations to an image before presenting it to the retinal input of the network. The perturbation type and magnitude are stored as noted below in successive locations ( $\mathrm{L}$ values) of TEMPERTURB $(1, \mathrm{~L})$ and TEMPERTURB $(2, \mathrm{~L})$.

TEMPERTURB $(1, \mathrm{~L}=1-->$ NLABELPARM $)$

Perturbation type $(1->6)$ for the Lth image perturbation.

TEMPERTURB(2,L=1 --> NLABELPARM)

Perturbation value for the $L$ th image perturbation.

Although we have not described every system array, the omitted ones serve in such obvious (or trivial) ways that their inclusion here seemed unnecessary. Likewise, we have not been 
exhaustive in the descriptions we have given. Our intent has been to include sufficient material to guide the interested reader toward his own path of discovery.

\section{APPLICATION OF LILARTI METHOD TO NON-DESTRUCTIVE EVALUATION}

One of the most difficult problems associated with the operation of nuclear power generating plants is assessment of the condition of the steam generator tubes. Measurements made during off-line periods must be of predictive power sufficient to permit anticipation of potential flaw-induced tube failures and so make possible such remedial action as may be required to prevent those failures. At present, the scheme principally utilized for making the required measurements incorporates the several techniques of eddy-current signal signature analysis ${ }^{11}$. Although amenable to some analytical data reduction methods ${ }^{12}$, eddy-current signal assessment, as presently employed, depends heavily on the image analysis capabilities of a well trained human operator. Results therefore are not only operator dependent, but are obtained at considerably less than optimum speed.

As traditionally performed, the eddy-current measurement of a steam generator tube involves the scanning of extended tube sections with probes bearing one or more signal-inducing coils and a sense coil (which may be the signal coil itself). The data extracted from the sense coil output are usually presented in the form of two-dimensional impedance plots which must be continuously monitored by human operators trained to recognize the small signal perturbations indicative of flaws. Often, interfering signals unrelated to any material defect or property of importance obscure those of interest and yield false indications which must be recognized as such. It is not surprising, therefore, that the process of flaw assessment, however well supported by algorithmic data analysis methods, is ultimately dependent on the judgement and interpretative skills of highly-trained (yet sometimes fallible) human signal evaluators. When we first began to consider the problem of automating eddy-current signal interpretation, our hope was to utilize the image-related capabilities of the LILARTI neural network method in such a way as to combine something of the human's interpretative abilities with the accuracy, consistency and absence of fatigue-induced human error characteristic of the computer. We note in Table 3 below the principal goals we set for the eddy-current signal evaluation studies, then describe briefly the system developed for application to the problem, and finally note some of the results.

It bears remarking upon here that, despite our continuing assertion that the best tasks for artificial neural networks (for those of the LILARTI sort, at least) are those which capture the least computational of human tasks, we attempted, with the eddy-current system, to address the most unlikely of the tasks important to eddy-current signal evaluation. Thus, whereas the human evaluator learns to identify regions of the impedance plot potentially indicative of an anomaly and submits the relevant data for computational analysis and final flaw assessment, our insistence that the neural analysis be derived completely from "visual cues" led us to attempt to construct and train a network capable of taking on the whole problem, identification of potentially relevant image regions and anomaly evaluation. That we met with any success at all seems, in retrospect, quite surprising. 
Table 3. Initial Goals for Neural Network-Based Eddy-Current Analysis System

(1) Capability for dealing directly, as does the human eddy-current signal evaluator, with image-oriented flaw-induced signals (in contradistinction to other neural network approaches which rely for their operation on a data reduction step which eliminates all vestiges of the image character of the original data)

(2) Capacity for describing the characteristics (depth, nature, and extent of perturbing influences) of detected flaws in terms of simple natural language parameters

(3) Embodiment of biologically oriented LILARTI neural network methodology

(4) Improved performance in terms of speed and freedom from human "bias"

(5) PC supportable

A second point which must be emphasized here concerns the specific details of the networks employed for the eddy-current studies. When we began we had just completed development of systems capable of angle, scale, and position independent image recognition and description. These systems involved the very large (and very slow!) networks of the general type represented by Figure 1 of Section II. Because we were aware that position, scale, and angle dependence (as opposed to the independence we had so carefully provided for) were important in the eddy-current context, we simplified the network in some important (and, to us, painful) ways. As a result of these modifications, the neural connectivity for the earliest NDE studies resembled more nearly than we would have liked that of a classical bi-directional associative memory. We did not, of course, abandon the biologically motivated training rules and neural representations which had from the beginning characterized the LILARTI systems. However, much that would have been done with more competent computing equipment was eliminated from consideration.

One simplification which we saw we would have to effect in one way or another involved the large disparity between the size of a typical eddy-current image and the size of images we could practicably process with a LILARTI system. Figure 5 illustrates the general characteristics of a typical eddy-current signal as it would appear before processing. Presented to the human evaluator at a resolution of about 1000 by 1000 pixels, the pattern represents the path in the complex (impedance) plane resulting from the motion of an eddy-current probe along a steam genera'or tube bore. In this case, the signal represents the presence of a defect near a tube support and reflects the motion of the probe past, first, the support and, subsequently, the defect. Although we had explored some LILARTI systems with 101 pixel square retinas, we knew that, even at this modest size, we would never be able to achieve the processing speed required for training several hundred eddy-current patterns.

Figures 6 and 7 illustrate the essential characteristics of the method we devised for reducing the number of "image" pixels in initially presented eddy-current data to a manageable value, 
yet retaining something of the inherent image character of the original eddy-current signal. It will prove expedient to divide our discussion into two parts, the first concerned only with a single frequency signal without the non-linear polar scale expansion which is generally applied near the origin, the second presenting the two signals usually applied to the network and illustrating the non-linear expansion.

In the leftmost portion of Figure 6 is displayed a typical eddy-current signal as it would appear at the resolution (about 1000 by 1000 pixels) usually available to the human signal evaluator. Upon this signal we imagine that three grids (here 11 pixels square) are superimposed, as illustrated by the central column of squares in the figure. For each of these grids we define a different rule. In effect, the three rules shall specify three different ways of examining the original image. All the rules will be expressed in terms of what we shall call a "line-crossing", the transit through a grid element by the path

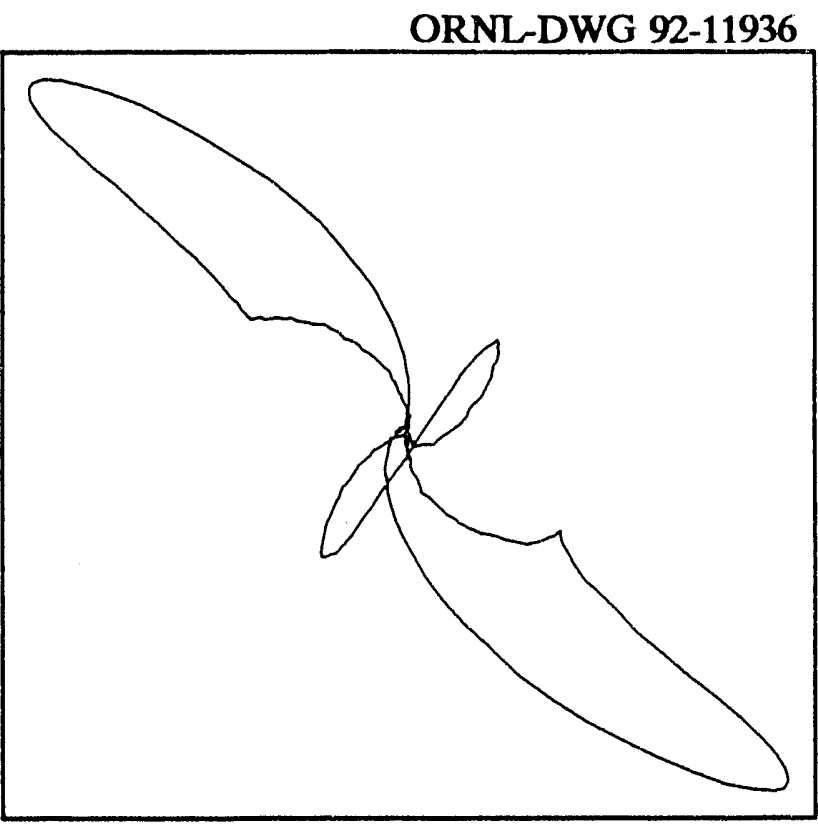

Fig. 5. $100 \mathrm{KHz}$ scan of $20 \%$ OD defect $1.1^{\prime \prime}$ from center of Fe deposit. defining the original image. Each rule will be, in effect, a different count of these line crossings. Thus, the first rule will be satisfied if the path crosses a grid element once and only once, the second if the path crosses a grid element twice and only twice, and the third if coordinate origin, make difficult the direct comparison of the illustrated initial and reduced images.the path crosses a grid element three or more times. The results of applying the three rules to the original image are illustrated by the righthand column of squares in the figure.

Figure 7 illustrates the application of the just described method to real eddy-current signals. The upper and lower halves (or rows) of the illustration depict the reduction process as applied at the two eddy-current signal frequencies employed for most of our tests (100 and $500 \mathrm{KHz}$ ). As described in the previous paragraph, the presentation for each frequency is further divided into four square regions (or cells). The raw eddy-current data, at an initial resolution of 1000 by 1000 pixels, are in each case represented by the figures in the left-rnost cells. Subsequent cells in each row are 11 by 11 pixels in size. The data reduction process may here be imagined as a set of six mappings (three for each frequency) of the 1000 by 1000 pixel data onto the six 11 by 11 grids which together form the 22 by 33 pixel "retina" serving as the input for the network's "visual" sense. A pixel of one of the 11 by 11 cells is set if and only if the condition which it represents is satisfied in the (approximately) 91 by 91 pixel region of the initial image which it represents. The conditions, as noted in the previous paragraph, involve "line crossings", the transit of the trajectory in the complex impedance plane through the region represented by a reduced pixel. As already noted, in the first pair of image-reducing cells (i.e., the two cells comprising the first column past the initial data presentations), an "on" pixel represents the one-time (and one-time only) crossing of the 


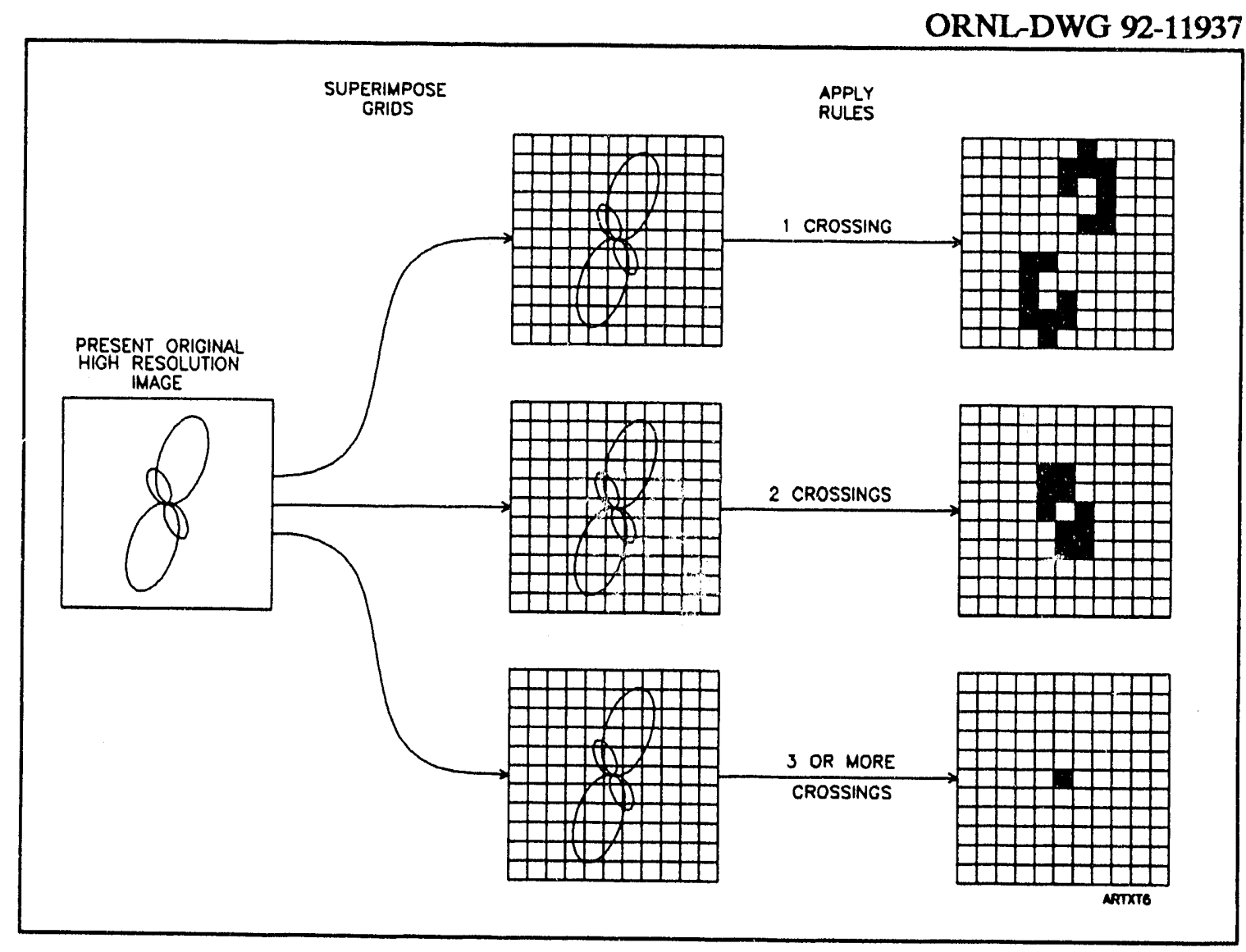

Fig. 6. Eddy-current image decomposition scheme.

corresponding region in the presented data by the signal "path". In the second pair of reducing cells, an "on" pixel represents the twice (and twice-only) crossing. An "on" pixel in the third cell pair denotes three or more crossings. It should be noted that the effects of the non-linear polar scale expansion noted earlier, introduced to enhance detail near the coordinate origin, make difficult the direct comparison of the illustrated initial and reduced images.

As artificial as this approach may appear at first sight, far less information is $1 \mathrm{r} \cdot \mathrm{t}$ by the compression process than might be imagined. For those examples which we have examined directly, it is the case that raw eddy-current images which the human assesses as similar result in sensibly similar representations in the abstracted format and images which are considered distinct lead to similarly distinct representations. For our purposes, the principal advantage conferred by this curious approach lies in the fact that, although the retina (at 22 by 33) remains rather large, the characteristic system connection density is defined only in terms of the six 11 by 11 retinal subregions (or, to be consistent with earlier usage, "subsheets") and the much smaller neural sheets which these serve. In the earliest eddy-current systems, those based on simplified versions of the original LILARTI, there were in some cases as few as six 11 by 11 intermediated levels driven by the six retinal regions and three intermediate levels ( 3 by 1,5 by 1 and 15 by 1 pixels in size respectively) driven by the "keyboard" inputs, the inputs at which the data describing parameter values were supplied. Thus, aside from the 


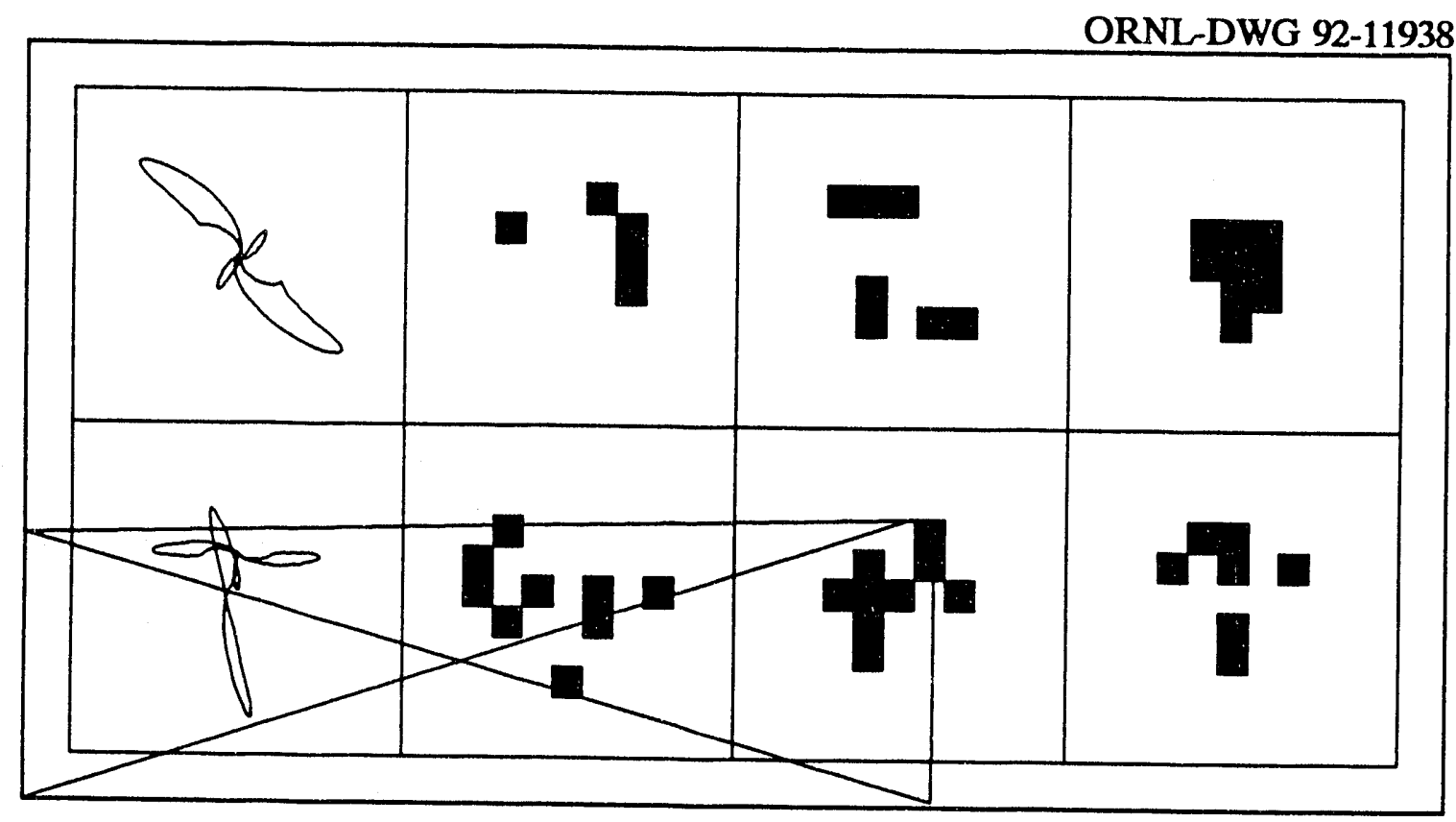

Fig. 7. Decomposition method applied to real eddy-current signals.

retinal neurons, some of the early eddy-current systems contained fewer than 700 trainably connected nodes. The results presented at the end of this section were obtained with just such a simple network. Nevertheless, these results have since been duplicated in accuracy in shorter training time (a factor of 8 to 10) with a much smaller LILARTI (fewer than 100 trainably connected neurons) of the newer variety discussed in Section III.

Before we present representative results for the eddy-current studies we must note a few important points concerning network training. We begin with a brief description of the method of selecting the training set (the collection of eddy-current images and associated descriptive parameters in terms of which the network will learn the relations between patterns and descriptions) from some complete eddy-current database. We wanted to avoid the possibility that the "hand-selection" method which we had earlier employed might introduce unintentional bias into the training process. Thus, the relevant code was arranged to choose, within some specified small error, a subset of a complete database the members of which subset comprise a user-indicated fraction of the whole. Consistency within a series of training sessions involving the same fractional part of the whole is ensured as a consequence of the method of random number generation. However, no attempt was made to guarantee an "even" distribution of the randomly selected training images nor to ensure the same distribution form for pairs of training sets representing different fractions of the whole database. The flexibility this selection process confers has an unfortunate concomitant which arises from the fact that for some parameter combinations small variations in one parameter value can markedly affect the characteristics of the associated eddy-current image whereas for another parameter combination the same small variation might have almost no observable effect. Thus, changing the training set fraction by as little as one percent can, because the number of "difficult image pairs" may change, have dramatic effects on system training performance. In order to minimize the effects on training of attempting to force learning of what amounted to very nonlinear, and apparently inconsistent, relationships between certain 
combinations of image characteristics and the associated parametric descriptions, we adjusted the training code so that small errors encountered during the testing phase of the training process would be tolerated. For all of the cases relevant to the present results, the accepted error was either 19 percent or 20 percent. It is worth noting that when the same eddy-current data were presented to the new LILARTI it proved possible to tighten the error limit by at least a factor of two and yet realize substantially equivalent trained behaviour.

As one last important point, it should be noted that the human signal evaluator, because he can observe the evolution of the impedance plot (as an oscilloscopic image), can make use of the "temporal" information inherent in the trace path to facilitate the process of disentangling the significances of the various signal contributions. Because the data are presented to the neural network as a static image, this considerable advantage is denied it. Nevertheless, for the data sets examined to date, it appears that the trained performance of the LILARTI networks at least equals that of a Level II eddy-current inspector. Whether or not this level of performance can be sustained in the presence of data characterized by noise and greater complexity remains to be determined.

The experimental results discussed below reflect the performance of the simplified LILARTI neural network system noted in Section II in the context of flaw data obtained under laboratory conditions.

The apparatus employed for data acquisition closely modeled the configuration of Figure 8. Illustrated here is the standard method of mounting a steam generator tube in the tube sheet and tube support. The principle flaw types are illustrated as well. IGA refers to inter-granular attack, IGSCC to inter-granular stress corrosion cracking. Copper and magnetite are two of the major depositing materials whose presence must be detected during tube examination. The term "Probe Wobble" refers to a (generally lateral) displacement of the eddy-current probe and is a motion whose effects must be eliminated in order to obtain accurate property data. We note that the general term "flaw" which we have used to this point will, in our subsequent remarks, be replaced by the terms "defect" and "artifact" (or "perturbation"). The former will refer primarily to holes in the steam generator tubing, the latter either to deposits on the tubing or to the presence of the supporting tube sheet.

The specific test piece from which the experimental data were acquired was a

ORNL-DWG 92-11939

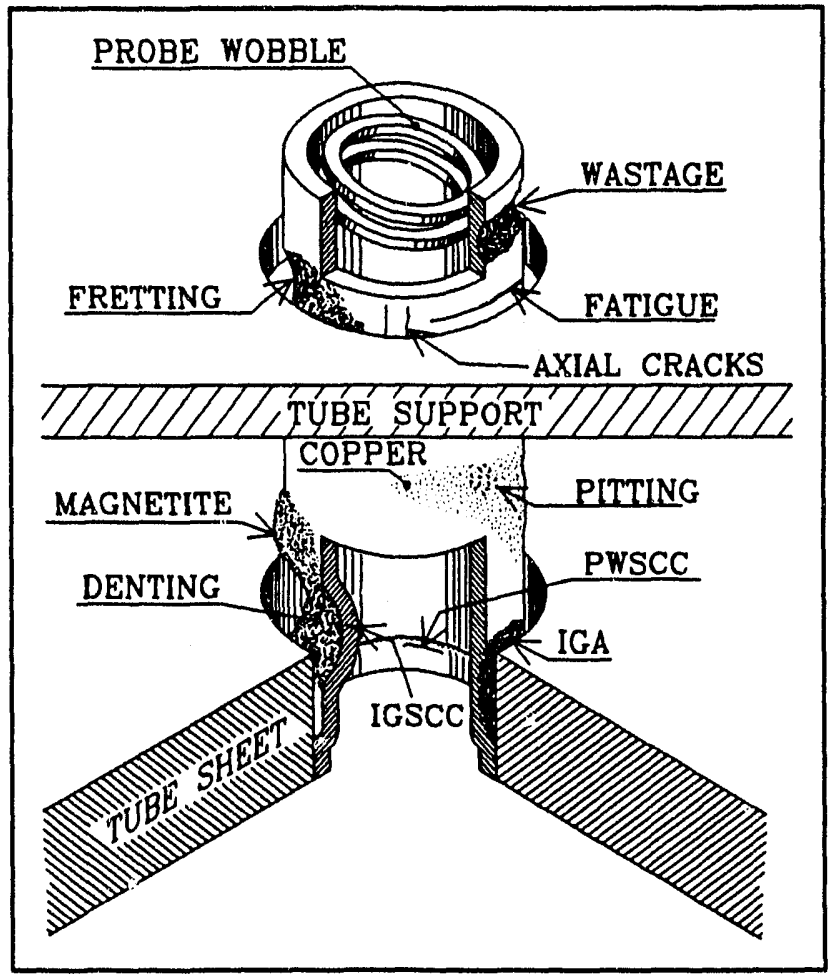

Fig. 8. Steam generator tube in support. 
section of steam generator tubing (50 mil. wall) into and along which tube defects (flatbottomed O.D. holes of five evenly spaced depths ranging from 10 mil to 50 mil, the latter a through-hole) and perturbations (or, as they are subsequently referred to, artifacts) of known types (ferrite deposits, copper deposits, and presence of tube support) had been introduced. Since our principal concern was the demonstration that a suitably trained artificial neural network system could evaluate defect depth in the presence of the noted artifacts and at the same time identify the dominant artifact type and the distance of the defect from it, we prepared the test piece in such a way that the flat-bottomed holes could be positioned at carefully controlled distances from, in turn, artifacts of each of the three types. Artifact-todefect distances were set in .1 inch increments. The eddy-current probe drive and dataaccumulation processes were both automated and under the control of a PC. Probe motion was defined in terms of 10 mil. steps.

Tables 4 through 25 summarize results obtained with an early LILARTI system (Section II). As noted above, an equivalent level of trained performance has been achieved with a new LILARTI system (Section III) of much smaller size and in much less time. Except as denoted by the symbol "19x19", system "visual" resolution was defined by the six 11 by 11 pattern abstractions illustrated by Figure 7 . Of the two groups into which these tables are divided, the first (Tables 4 through 14) represents the trained behaviour for all artifact types. Because the network exhibited completely error-free response to artifact type, it seemed reasonable to compare sensitivity to defect depth and distance from artifact as a function of artifact type. These results are summarized in Tables 15 through 25 . For both groups of tables, the tabulated values represent the trained performance for a training set whose size is the indicated fraction of the complete 225 example data set. Each training set "element" is of the general form illustrated by Figure 7. Training time for the various training sets ranged from one day to five days when the code was executed on a $25 \mathrm{MHz}$, Weitek-enhanced $486 \mathrm{PC}$ clone. The indicated "resolution" values refer, as noted above, to the number of distinguishable identifications for the relevant parameter. These figures should be interpreted in the context of the maximum values for the associated parameters $(50 \mathrm{mils}$. for defect depth, 1.5 inches for distance from artifact) The identification of artifact type (tube support (1), magnetite deposit (2), copper deposit (3)) was, as noted above, without error in all cases. Somewhat less impressive, but still surprising considering the relatively small training set size, is the accuracy with which the depth of the defect is assessed. The apparently dismal results for distance of defect from nearest artifact must be understood in the context of the data accumulation method. During the accumulation process, probe motion was continued to a range which had been determined such that, for the strongest artifact signal, no vestige of signal due to that artifact remained visible to the monitoring human. Because signal elements due specifically to the presence of an artifact decrease in amplitude with distance they are increasingly poor indicators of artifact range as range increases.

In the interest of completeness, we note what amounts to a neural "wiring" error in the NDE network and its effects on the data analysis process. It has been fairly universally the case for LILARTI systems that data representing network response to stimuli be made available at a set of neural regions established by statements in the LILARTI code. It is from the status of these program-specified regions (or, better, from the set of neural outputs at these regions) 
that the TUTOR determines the nature of training and testing errors. In the network whose behaviour we describe here, the (non-trainable) wiring leading to these response-defining regions had been so arranged as to resolve such conflicts as might exist when convergence to a unique final state failed to obtain. As a result of the insufficient strengths assigned to the associated connections (the wiring error), unambiguous final state information was, despite our good intentions, not always available at these highest level regions. Thus, the automated TUTOR testing routine was at times unable to detect responses which the system had given to test data. Nevertheless, because the outputs of those lower levels responsible for driving the program-specified output regions were accessible to inspection, it was possible to determine a system response. The results presented here derive in some cases, therefore, from assessments made of these "once-removed" neural regions. For practical purposes, the only byproduct of the error was the time lost to "manual" evaluation. When resolution of ambiguous responses was required, we erred always on the side of pessimism. Thus, the results presented here reflect a worst-case assessment of system performance.

In the following, trained performance is tabulated for the three trained-for parameters (artifact type, defect depth, and distance from defect to nearest artifact). As already suggested, entries in the "Resolution" column refer, in reverse order, to the number of distinct parameter values in the training data for the corresponding parameter and to the smallest inter-value increment actually employed. In all cases, there were three artifact types, all of which were trained for, five defect depths, all of which were trained for, and fifteen distances from nearest artifact, all of which were trained for. An entry in the "Percent Correctly Identified" column denotes the percentage of test cases for which the corresponding parameter was correctly assessed in value. An entry in the "Percent Within One RW" column denotes the percentage of test cases for which the corresponding parameter was determined to within one resolution width (RW) of the correct value. An entry in the "Percent Worse Than One RW" column denotes the percentage of test cases for which the measured value of the corresponding parameter lay more than one resolution width from the correct value.

The apparently surprising broad dependence of trained behaviour on the degree of training can be understood in the context of potentially competing influences. Although a full analysis in terms of the contributing factors is incomplete, it may be noted that, on the one hand, training on increasingly large fractions of the complete data set refines the trained state of system wiring and should be expected to enhance the interpolative response capability. On the other, the image reduction scheme is incapable of resolving the correspondingly more subtle inter-image differences which the larger training fractions imply. Moreover, the 80 (or 81) percent factor which defines image similarity is more crude than the nearest-neighbour image differences in some cases. For comparison purposes, results of two runs (at $75 \%$ and $90 \%$ data sets) at an image reduction to 19 by 19 are presented. Note that, although the results for the $90 \%$ training set are noticeably better than the corresponding results for the 11 by 11 run, those for the $75 \%$ set are worse. 
Table 4. Trained performance for $60 \%$ training set

\begin{tabular}{lcccc}
\hline & Resolution & $\begin{array}{c}\% \\
\text { correctly } \\
\text { identified }\end{array}$ & $\begin{array}{c}\% \\
\text { within } \\
1 \text { RW }\end{array}$ & $\begin{array}{c}\% \\
\text { worse than } \\
\text { 1 RW }\end{array}$ \\
\hline $\begin{array}{l}\text { Artifact } \\
\text { identification }\end{array}$ & $(1 / 3)$ & 100 & 0 & 0 \\
$\begin{array}{l}\text { Defect depth } \\
\text { identification }\end{array}$ & $(1 / 5)$ & 72 & 14 & 14 \\
$\begin{array}{l}\text { Distance from } \\
\text { defect to nearest } \\
\text { artifact }\end{array}$ & $(1 / 15)$ & 15 & 49 & 36 \\
\hline
\end{tabular}

Table 5. Trained performance for $60 \%$ training set even distribution run

\begin{tabular}{lcccc}
\hline & Resolution & $\begin{array}{c}\% \\
\text { correctly } \\
\text { identified }\end{array}$ & $\begin{array}{c}\% \\
\text { within } \\
\text { 1 RW }\end{array}$ & $\begin{array}{c}\% \\
\text { worse than } \\
\text { 1 RW }\end{array}$ \\
\hline $\begin{array}{l}\text { Artifact } \\
\text { identification }\end{array}$ & $(1 / 3)$ & 100 & 0 & 0 \\
$\begin{array}{l}\text { Defect depth } \\
\text { identification }\end{array}$ & $(1 / 5)$ & 73 & 7 & 20 \\
$\begin{array}{l}\text { Distance from } \\
\text { defect to nearest } \\
\text { artifact }\end{array}$ & $(1 / 15)$ & 22 & 42 & 36 \\
\hline
\end{tabular}

Table 6. Trained performance for $65 \%$ training set

\begin{tabular}{lcccc}
\hline & Resolution & $\begin{array}{c}\% \\
\text { correctly } \\
\text { identified }\end{array}$ & $\begin{array}{c}\% \\
\text { within } \\
1 \text { RW }\end{array}$ & $\begin{array}{c}\% \\
\text { worse than } \\
1 \text { RW }\end{array}$ \\
\hline $\begin{array}{l}\text { Artifact } \\
\text { identification }\end{array}$ & $(1 / 3)$ & 100 & 0 & 0 \\
$\begin{array}{l}\text { Defect depth } \\
\begin{array}{l}\text { Distance from } \\
\text { defect to nearest }\end{array}\end{array}$ & $(1 / 5)$ & 73 & 7 & 20 \\
artifact & 13 & 38 & 49 \\
\hline
\end{tabular}


Table 7. Trained performance for $70 \%$ training set

\begin{tabular}{lcccc}
\hline & Resolution & $\begin{array}{c}\% \\
\text { correctly } \\
\text { identified }\end{array}$ & $\begin{array}{c}\% \\
\text { within } \\
1 \mathrm{RW}\end{array}$ & $\begin{array}{c}\% \\
\text { worse than } \\
\text { 1 RW }\end{array}$ \\
\hline $\begin{array}{l}\text { Artifact } \\
\text { identification }\end{array}$ & $(1 / 3)$ & 100 & 0 & 0 \\
$\begin{array}{l}\text { Defect depth } \\
\text { identification }\end{array}$ & $(1 / 5)$ & 73 & 10 & 17 \\
$\begin{array}{l}\text { Distance from } \\
\text { defect to nearest } \\
\text { artifact }\end{array}$ & $(1 / 15)$ & 17 & 43 & 40 \\
\hline
\end{tabular}

Table 8. Trained performance for $75 \%$ training set

\begin{tabular}{lcccc}
\hline & Resolution & $\begin{array}{c}\% \\
\text { correctly } \\
\text { identified }\end{array}$ & $\begin{array}{c}\% \\
\text { within } \\
\text { 1 RW }\end{array}$ & $\begin{array}{c}\% \\
\text { worse than } \\
\text { 1 RW }\end{array}$ \\
\hline $\begin{array}{l}\text { Artifact } \\
\text { identification }\end{array}$ & $(1 / 3)$ & 100 & 0 & 0 \\
$\begin{array}{l}\text { Defect depth } \\
\text { identification }\end{array}$ & $(1 / 5)$ & 80 & 7 & 13 \\
$\begin{array}{l}\text { Distance from } \\
\text { defect to nearest } \\
\text { artifact }\end{array}$ & $(1 / 15)$ & 18 & 50 & 32 \\
\hline
\end{tabular}

Table 9. Trained performance for $75 \%$ training set (19x19)

\begin{tabular}{lcccc}
\hline & Resolution & $\begin{array}{c}\% \\
\text { correctly } \\
\text { identified }\end{array}$ & $\begin{array}{c}\% \\
\text { within } \\
\text { 1 RW }\end{array}$ & $\begin{array}{c}\% \\
\text { worse than } \\
\text { 1 RW }\end{array}$ \\
\hline $\begin{array}{l}\text { Artifact } \\
\text { identification }\end{array}$ & $(1 / 3)$ & 100 & 0 & 0 \\
$\begin{array}{l}\text { Defect depth } \\
\text { identification }\end{array}$ & $(1 / 5)$ & 71 & 10 & 19 \\
$\begin{array}{l}\text { Distance from } \\
\text { defect to nearest } \\
\text { artifact }\end{array}$ & $(1 / 15)$ & 20 & 51 & 29 \\
\hline
\end{tabular}


Table 10. Trained performance for $80 \%$ training set

\begin{tabular}{lcccc}
\hline & Resolution & $\begin{array}{c}\% \\
\text { correctly } \\
\text { identified }\end{array}$ & $\begin{array}{c}\% \\
\text { within } \\
\text { i RW }\end{array}$ & $\begin{array}{c}\% \\
\text { worse than } \\
1 \text { RW }\end{array}$ \\
\hline $\begin{array}{l}\text { Artifact } \\
\text { identification }\end{array}$ & $(1 / 3)$ & 100 & 0 & 0 \\
$\begin{array}{l}\text { Defect depth } \\
\text { identification }\end{array}$ & $(1 / 5)$ & 72 & 11 & 17 \\
$\begin{array}{l}\text { Distance from } \\
\text { defect to nearest } \\
\text { artifact }\end{array}$ & $(1 / 15)$ & 17 & 48 & 35 \\
\hline
\end{tabular}

Table 11. Trained performance for $85 \%$ training set

\begin{tabular}{lcccc}
\hline & Resolution & $\begin{array}{c}\% \\
\text { correctly } \\
\text { identified }\end{array}$ & $\begin{array}{c}\% \\
\text { within } \\
1 \text { RW }\end{array}$ & $\begin{array}{c}\% \\
\text { worse than } \\
\text { 1 RW }\end{array}$ \\
\hline $\begin{array}{l}\text { Artifact } \\
\text { identification }\end{array}$ & $(1 / 3)$ & 100 & 0 & 0 \\
$\begin{array}{l}\text { Defect depth } \\
\text { identification }\end{array}$ & $(1 / 5)$ & 76 & 9 & 15 \\
$\begin{array}{l}\text { Distance from } \\
\text { defect to nearest } \\
\text { artifact }\end{array}$ & $(1 / 15)$ & 21 & 38 & 41 \\
\hline
\end{tabular}

Table 12 Trained performance for $90 \%$ training set

\begin{tabular}{lcccc}
\hline & Resolution & $\begin{array}{c}\% \\
\text { correctly } \\
\text { identified }\end{array}$ & $\begin{array}{c}\% \\
\text { within } \\
\text { 1 RW }\end{array}$ & $\begin{array}{c}\% \\
\text { worse than } \\
\text { 1 RW }\end{array}$ \\
\hline $\begin{array}{l}\text { Artifact } \\
\text { identification }\end{array}$ & $(1 / 3)$ & 100 & 0 & 0 \\
$\begin{array}{l}\text { Defect depth } \\
\text { identification }\end{array}$ & $(1 / 5)$ & 79 & 8 & 13 \\
$\begin{array}{l}\text { Distance from } \\
\text { defect to ncarcsi } \\
\text { artifact }\end{array}$ & $(1 / 15)$ & 8 & 50 & 42 \\
\hline
\end{tabular}


Table 13. Trained performance for $90 \%$ training set (19x19)

\begin{tabular}{lcccc}
\hline & Resolution & $\begin{array}{c}\% \\
\text { correctly } \\
\text { identified }\end{array}$ & $\begin{array}{c}\% \\
\text { within } \\
\text { 1 RW }\end{array}$ & $\begin{array}{c}\% \\
\text { worse than } \\
\text { 1 RW }\end{array}$ \\
\hline $\begin{array}{l}\text { Artifact } \\
\text { identification }\end{array}$ & $(1 / 3)$ & 100 & 0 & 0 \\
$\begin{array}{l}\text { Defect depth } \\
\text { identification }\end{array}$ & $(1 / 5)$ & 90 & 5 & 5 \\
$\begin{array}{l}\text { Distance from } \\
\text { defect to nearest } \\
\text { artifact }\end{array}$ & $(1 / 15)$ & 30 & 50 & 20 \\
\hline
\end{tabular}

Table 14. Trained performance for $95 \%$ training set

\begin{tabular}{lcccc}
\hline & Resolution & $\begin{array}{c}\% \\
\text { correctly } \\
\text { identified }\end{array}$ & $\begin{array}{c}\% \\
\text { within } \\
\text { 1 RW }\end{array}$ & $\begin{array}{c}\% \\
\text { worse than } \\
\text { 1 RW }\end{array}$ \\
\hline $\begin{array}{l}\text { Artifact } \\
\text { identification }\end{array}$ & $(1 / 3)$ & 100 & 0 & 0 \\
$\begin{array}{l}\text { Defect depth } \\
\text { identification }\end{array}$ & $(1 / 5)$ & 70 & 10 & 20 \\
$\begin{array}{l}\text { Distance from } \\
\text { defect to nearest } \\
\text { artifact }\end{array}$ & $(1 / 15)$ & 10 & 40 & 50 \\
\hline
\end{tabular}

In the following, the errors in defect depth assessment (error type 2) and defect-to-artifact distance (error type 3) are tabulated as functions of artifact type. Again, artifact type 1 refers to the tube support, 2 to magnetite deposits, and 3 to copper deposits. The entries under the heading "\#" refer to the number of examples of the indicated artifact type in the randomly selected training set. The entries under "Error" denote defect depth measurements (2) and distance measurements (3). Number/(\%) refers to number and the corresponding percentage of test cases falling in the indicated error bin. These latter are, as noted, the "correct" bin, the "1 RW error" bin, and the "everything else" bin. 
Table 15. Distribution of observed testing errors by artifact type data set (\%) 60

\begin{tabular}{cccccc}
\hline $\begin{array}{c}\text { Artifact } \\
\text { type }\end{array}$ & $\#$ & $\begin{array}{c}\text { Error } \\
(2 / 3)\end{array}$ & $\begin{array}{c}\text { Number/(\%) } \\
\text { correct }\end{array}$ & $\begin{array}{c}\text { Number/(\%) } \\
\text { within } \\
1 \mathrm{RW}\end{array}$ & $\begin{array}{c}\text { Number/(\%) } \\
\text { worse than } \\
1 \mathrm{RW}\end{array}$ \\
\hline 1 & 33 & 2 & $23 / 70$ & $5 / 15$ & $5 / 15$ \\
& & 3 & $5 / 15$ & $15 / 46$ & $13 / 39$ \\
2 & 31 & 2 & $26 / 84$ & $3 / 10$ & $2 / 6$ \\
& & 3 & $3 / 10$ & $17 / 55$ & $11 / 35$ \\
3 & 27 & 2 & $16 / 59$ & $5 / 19$ & $6 / 22$ \\
& & 3 & $5 / 19$ & $13 / 48$ & $9 / 33$ \\
\hline
\end{tabular}

Table 16. Distribution of observed testing errors by artifact type data set (\%) 60 (smooth)

\begin{tabular}{cccccc}
\hline $\begin{array}{c}\text { Artifact } \\
\text { type }\end{array}$ & $\#$ & $\begin{array}{c}\text { Error } \\
(2 / 3)\end{array}$ & $\begin{array}{c}\text { Number/(\%) } \\
\text { correct }\end{array}$ & $\begin{array}{c}\text { Number/(\%) } \\
\text { within } \\
1 \mathrm{RW}\end{array}$ & $\begin{array}{c}\text { Number/(\%) } \\
\text { worse than } \\
1 \mathrm{RW}\end{array}$ \\
\hline 1 & 31 & 2 & $25 / 81$ & $2 / 6$ & $4 / 13$ \\
& & 3 & $8 / 26$ & $13 / 42$ & $10 / 32$ \\
2 & 27 & 2 & $19 / 70$ & $3 / 11$ & $4 / 19$ \\
& & 3 & $6 / 22$ & $9 / 33$ & $12 / 44$ \\
3 & 30 & 2 & $20 / 67$ & $1 / 3$ & $9 / 30$ \\
& & 3 & $5 / 17$ & $15 / 50$ & $10 / 33$ \\
\hline
\end{tabular}

Table 17. Distribution of observed testing errors by artifact type data set (\%) 65

\begin{tabular}{cccccc}
\hline $\begin{array}{c}\text { Artifact } \\
\text { type }\end{array}$ & $\#$ & $\begin{array}{c}\text { Error } \\
(2 / 3)\end{array}$ & $\begin{array}{c}\text { Number/(\%) } \\
\text { correct }\end{array}$ & $\begin{array}{c}\text { Number/(\%) } \\
\text { within } \\
1 \mathrm{RW}\end{array}$ & $\begin{array}{c}\text { Number/(\%) } \\
\text { worse than } \\
1 \mathrm{RW}\end{array}$ \\
\hline 1 & 24 & 2 & $15 / 63$ & $4 / 17$ & $5 / 20$ \\
& & 3 & $5 / 21$ & $6 / 25$ & $13 / 54$ \\
2 & 27 & 2 & $21 / 78$ & $4 / 15$ & $2 / 7$ \\
& & 3 & $3 / 11$ & $12 / 44$ & $12 / 44$ \\
3 & 27 & 2 & $19 / 70$ & $4 / 15$ & $4 / 15$ \\
& & & $2 / 7$ & $12 / 44$ & $13 / 48$ \\
\hline
\end{tabular}


Table 18. Distribution of observed testing errors by artifact type data set (\%) 70

\begin{tabular}{cccccc}
\hline $\begin{array}{c}\text { Artifact } \\
\text { type }\end{array}$ & \# & $\begin{array}{c}\text { Error } \\
(2 / 3)\end{array}$ & $\begin{array}{c}\text { Number/(\%) } \\
\text { correct }\end{array}$ & $\begin{array}{c}\text { Number/(\%) } \\
\text { within } \\
1 \mathrm{RW}\end{array}$ & $\begin{array}{c}\text { Number/(\%) } \\
\text { worse than } \\
1 \mathrm{RW}\end{array}$ \\
\hline 1 & 26 & 2 & $20 / 77$ & $1 / 4$ & $5 / 19$ \\
& & 3 & $6 / 23$ & $12 / 46$ & $8 / 31$ \\
2 & 25 & 2 & $17 / 68$ & $6 / 24$ & $2 / 8$ \\
& & 3 & $5 / 20$ & $9 / 36$ & $11 / 44$ \\
3 & 19 & 2 & $14 / 74$ & $0 / 0$ & $5 / 26$ \\
& & 3 & $1 / 5$ & $9 / 47$ & $9 / 47$ \\
\hline
\end{tabular}

Table 19. Distribution of observed testing errors by artifact type data set (\%) 75

\begin{tabular}{cccccc}
\hline $\begin{array}{c}\text { Artifact } \\
\text { type }\end{array}$ & $\#$ & $\begin{array}{c}\text { Error } \\
(2 / 3)\end{array}$ & $\begin{array}{c}\text { Number/(\%) } \\
\text { correct }\end{array}$ & $\begin{array}{c}\text { Number/(\%) } \\
\text { within } \\
1 \text { RW }\end{array}$ & $\begin{array}{c}\text { Number/(\%) } \\
\text { worse than } \\
1 \text { RW }\end{array}$ \\
\hline 1 & 18 & 2 & $17 / 94$ & $0 / 0$ & $1 / 5$ \\
& & 3 & $4 / 22$ & $11 / 61$ & $3 / 17$ \\
2 & 19 & 2 & $16 / 84$ & $1 / 5$ & $2 / 11$ \\
& & 3 & $3 / 16$ & $6 / 32$ & $10 / 52$ \\
3 & 19 & 2 & $12 / 63$ & $3 / 16$ & $4 / 21$ \\
& & 3 & $3 / 16$ & $11 / 58$ & $5 / 26$ \\
\hline
\end{tabular}

Table 20. Distribution of observed testing errors by artifact type data set (\%) 75 (19×19)

\begin{tabular}{cccccc}
\hline $\begin{array}{c}\text { Artifact } \\
\text { type }\end{array}$ & $\#$ & $\begin{array}{c}\text { Error } \\
(2 / 3)\end{array}$ & $\begin{array}{c}\text { Number/(\%) } \\
\text { correct }\end{array}$ & $\begin{array}{c}\text { Number/(\%) } \\
\text { within } \\
1 \mathrm{RW}\end{array}$ & $\begin{array}{c}\text { Number/(\%) } \\
\text { worse than } \\
1 \mathrm{RW}\end{array}$ \\
\hline 1 & 22 & 2 & $13 / 59$ & $4 / 18$ & $5 / 23$ \\
& & 3 & $4 / 18$ & $12 / 55$ & $6 / 27$ \\
2 & 19 & 2 & $17 / 84$ & $0 / 0$ & $2 / 11$ \\
& & 3 & $3 / 16$ & $10 / 53$ & $6 / 31$ \\
3 & 18 & 2 & $12 / 67$ & $2 / 11$ & $4 / 22$ \\
& & 3 & $5 / 28$ & $8 / 44$ & $5 / 28$ \\
\hline
\end{tabular}


Table 21. Distribution of observed testing errors by artifact type data set (\%) 80

\begin{tabular}{cccccc}
\hline $\begin{array}{c}\text { Artifact } \\
\text { type }\end{array}$ & \# & $\begin{array}{c}\text { Error } \\
(2 / 3)\end{array}$ & $\begin{array}{c}\text { Number/(\%) } \\
\text { correct }\end{array}$ & $\begin{array}{c}\text { Number/(\%) } \\
\text { within } \\
1 \mathrm{RW}\end{array}$ & $\begin{array}{c}\text { Number/(\%) } \\
\text { worse than } \\
1 \mathrm{RW}\end{array}$ \\
\hline 1 & 15 & 2 & $12 / 80$ & $1 / 6$ & $2 / 13$ \\
& & 3 & $4 / 27$ & $5 / 33$ & $6 / 40$ \\
2 & 13 & 2 & $11 / 85$ & $1 / 7$ & $1 / 7$ \\
& & 3 & $1 / 7$ & $7 / 54$ & $5 / 38$ \\
3 & 18 & 2 & $10 / 55$ & $3 / 17$ & $5 / 28$ \\
& & 3 & $3 / 17$ & $10 / 55$ & $5 / 28$ \\
\hline
\end{tabular}

Table 22. Distribution of observed testing errors by artifact type data set (\%) 85

\begin{tabular}{cccccc}
\hline $\begin{array}{c}\text { Artifact } \\
\text { type }\end{array}$ & $\#$ & $\begin{array}{c}\text { Error } \\
(2 / 3)\end{array}$ & $\begin{array}{c}\text { Number/(\%) } \\
\text { correct }\end{array}$ & $\begin{array}{c}\text { Number/(\%) } \\
\text { within } \\
1 \mathrm{RW}\end{array}$ & $\begin{array}{c}\text { Number/(\%) } \\
\text { worse than } \\
1 \mathrm{RW}\end{array}$ \\
\hline 1 & 18 & 2 & $13 / 72$ & $1 / 5$ & $4 / 22$ \\
& & 3 & $3 / 17$ & $8 / 44$ & $7 / 39$ \\
2 & 9 & 2 & $9 / 100$ & $0 / 0$ & $0 / 0$ \\
& & 3 & $2 / 22$ & $3 / 33$ & $4 / 44$ \\
3 & 6 & 2 & $3 / 50$ & $2 / 33$ & $1 / 17$ \\
& & 3 & $1 / 17$ & $2 / 33$ & $3 / 50$ \\
\hline
\end{tabular}

Table 23. Distribution of observed testing errors by artifact type data set (\%) 90

\begin{tabular}{cccccc}
\hline $\begin{array}{c}\text { Artifact } \\
\text { type }\end{array}$ & $\#$ & $\begin{array}{c}\text { Error } \\
(2 / 3)\end{array}$ & $\begin{array}{c}\text { Number/(\%) } \\
\text { correct }\end{array}$ & $\begin{array}{c}\text { Number/(\%) } \\
\text { within } \\
1 \mathrm{RW}\end{array}$ & $\begin{array}{c}\text { Number/(\%) } \\
\text { worse than } \\
1 \mathrm{RW}\end{array}$ \\
\hline 1 & 9 & 2 & $7 / 78$ & $0 / 0$ & $2 / 22$ \\
& & 3 & $1 / 11$ & $4 / 44$ & $4 / 44$ \\
2 & 10 & 2 & $8 / 80$ & $2 / 20$ & $0 / 0$ \\
& & 3 & $0 / 0$ & $5 / 50$ & $5 / 50$ \\
3 & 5 & 2 & $4 / 80$ & $0 / 0$ & $1 / 20$ \\
& & 3 & $1 / 20$ & $3 / 60$ & $1 / 20$ \\
\hline
\end{tabular}


Table 24. Distribution of observed testing errors by artifact type data set (\%) $90(19 \times 19)$

\begin{tabular}{cccccc}
\hline $\begin{array}{c}\text { Artifact } \\
\text { type }\end{array}$ & $\#$ & $\begin{array}{c}\text { Error } \\
(2 / 3)\end{array}$ & $\begin{array}{c}\text { Number/(\%) } \\
\text { correct }\end{array}$ & $\begin{array}{c}\text { Number/(\%) } \\
\text { within } \\
1 \mathrm{RW}\end{array}$ & $\begin{array}{c}\text { Number/(\%) } \\
\text { worse than } \\
1 \mathrm{RW}\end{array}$ \\
\hline 1 & 7 & 2 & $7 / 100$ & $0 / 0$ & $0 / 0$ \\
& & 3 & $3 / 43$ & $3 / 43$ & $1 / 14$ \\
2 & 10 & 2 & $8 / 80$ & $1 / 10$ & $1 / 10$ \\
& & 3 & $3 / 30$ & $4 / 40$ & $3 / 30$ \\
3 & 3 & 2 & $3 / 100$ & $0 / 0$ & $0 / 0$ \\
& 3 & $0 / 0$ & $3 / 100$ & $0 / 0$ \\
\hline
\end{tabular}

Table 25. Distribution of observed testing errors by artifact type data set (\%) 95

\begin{tabular}{cccccc}
\hline $\begin{array}{c}\text { Artifact } \\
\text { type }\end{array}$ & $\#$ & $\begin{array}{c}\text { Error } \\
(2 / 3)\end{array}$ & $\begin{array}{c}\text { Number/(\%) } \\
\text { correct }\end{array}$ & $\begin{array}{c}\text { Number/(\%) } \\
\text { within } \\
1 \mathrm{RW}\end{array}$ & $\begin{array}{c}\text { Number/(\%) } \\
\text { worse than } \\
1 \mathrm{RW}\end{array}$ \\
\hline 1 & 5 & 2 & $2 / 40$ & $1 / 20$ & $2 / 40$ \\
& & 3 & $0 / 0$ & $3 / 60$ & $2 / 40$ \\
2 & 3 & 2 & $3 / 100$ & $0 / 0$ & $0 / 0$ \\
& & 3 & $0 / 0$ & $0 / 0$ & $3 / 100$ \\
3 & 2 & 2 & $2 / 100$ & $0 / 0$ & $0 / 0$ \\
& & 3 & $1 / 50$ & $1 / 50$ & $0 / 0$ \\
\hline
\end{tabular}

Figures 9 and 10 are provided for the reader more comfortable with data presented in graphical form. Figure 9 includes the data of the first set of tables, Figure 10 those of the second.

On the basis of results to date, it is fair to suggest that we have demonstrated the feasibility of applying a neural net methodology based on biological rather than mathematical principles to the task of assessing the nature of material flaws reflected in eddy-current signals presented to the neural net, as they are to the human signal evaluator, in "visual" form. Although it is very likely that our approach is less efficient at the simple task of memorization than the more classically rooted systems which comprise the mainstream of neural net activity, the capabilities gained, particularly those relevant to the capacity for providing descriptions of presented signals (as opposed to the mere recognition that a presented signal may be like one which has been encountered before) appear to us substantially to outweigh the disadvantages deriving from the loss of speed, particularly as these latter may easily be overcome by the simple expedient of employing more sophisticated (albeit more expensive) processing hardware. The 
ORNL-DWG 92-11940

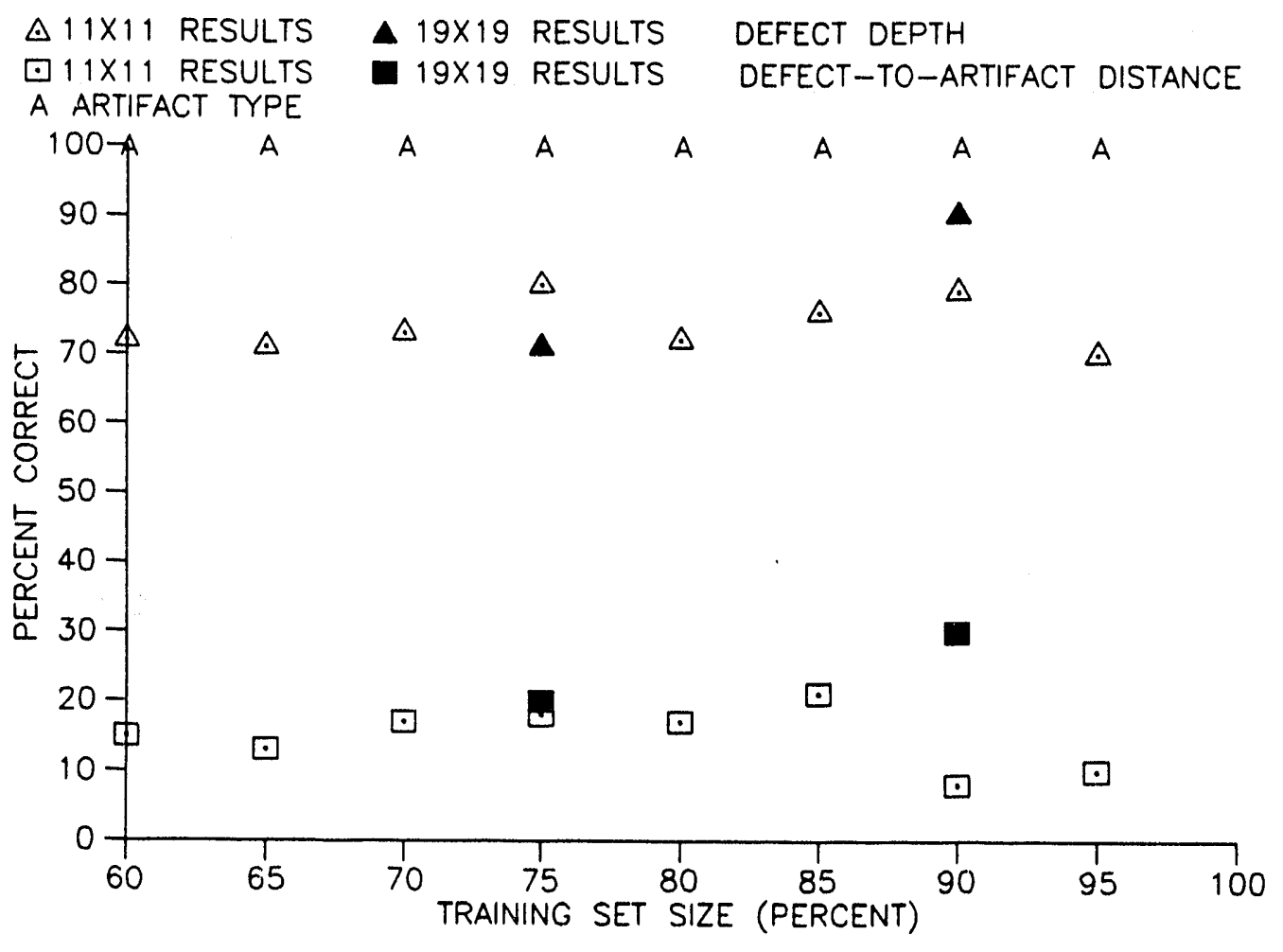

Fig. 9. Performance as a function of training set size: percent correctly identified. 


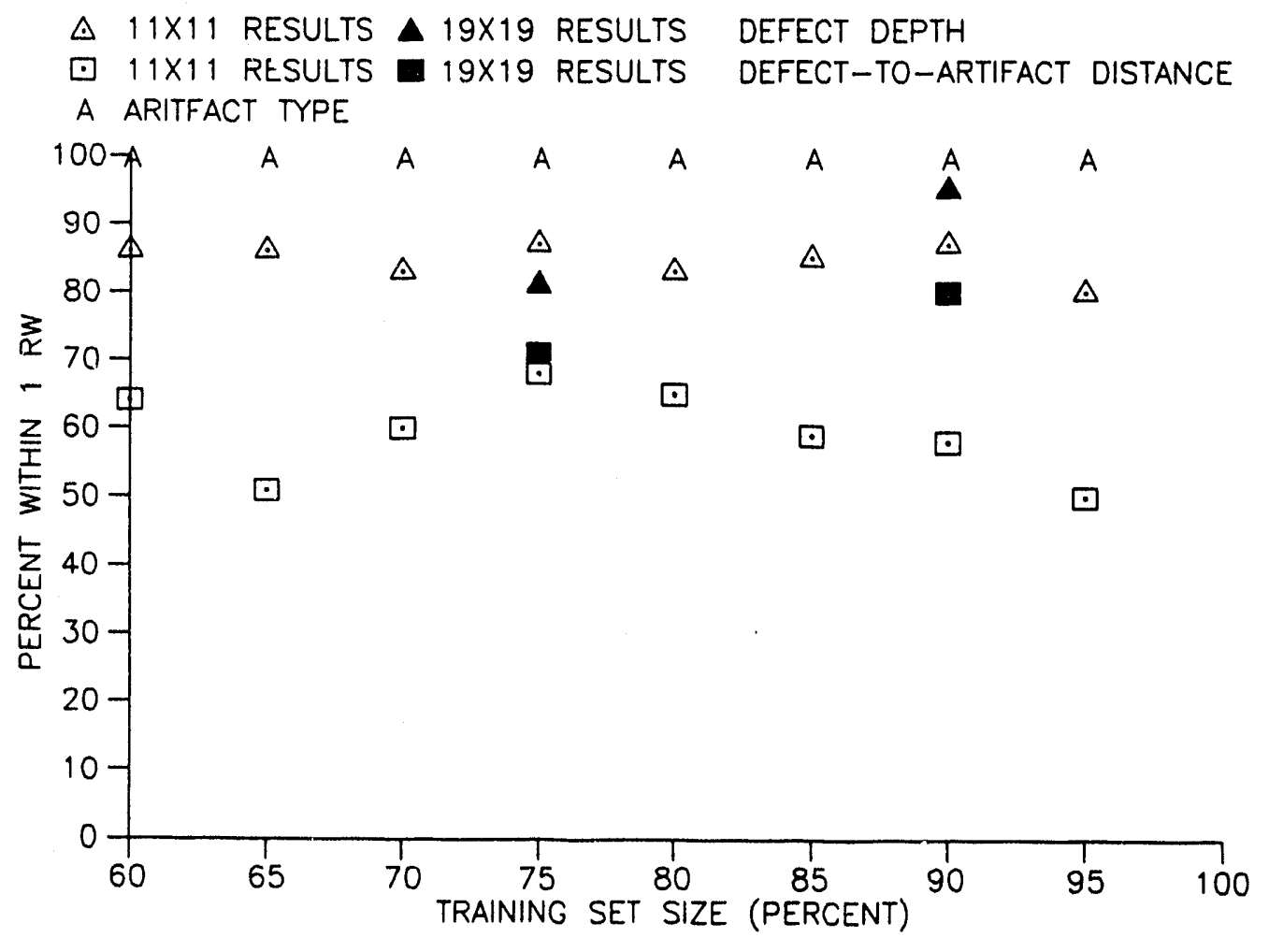

Fig. 10. Performance as a function of training set size: percent identified within $1 \mathrm{RW}$ error. 
full utility of the developing techniques will become clear only upon extension first to full eddy-current capability and subsequently into those considerably more difficult arenas of nondestructive evaluation characterized by higher dimensionality and time dependence.

\section{VI. "DIRECTIONS" FOR STUDIES}

In this section we discuss very briefly some of the applications to which the new LILARTI method might profitably be applied and note some of the research directions which those interested in the more cognitive aspects of artificial neural systems may wish to pursue. The code, which is available on request from the authors, can be readily modified by the interested reader to support any of these and, no doubt, many more as well.

As we consider the most logical extension of the neural network eddy-current assessment system we note that, despite the success of our present approach, optimum utilization is not made of the capabilities of the LILARTI scheme. Indeed, although we have made much of the "visual" aspects of the LILARTI networks, we are, in effect, requiring capabilities of the neural system which the human it is intended to emulate cannot perform without the assistance of more algorithmic techniques. The human efdy-current evaluator is not generally expected to derive complete defect and artifact specifications from the eddy-current images alone. The images serve, rather, as means for identifying those positions along a tube (or along some other substrate under test) at which something anomalous is detected. The human inspector, having noted these positions, subjects the raw data relevant only to them to one or another of several well known algorithmic analysis schemes (see, for example, Deeds, Dodd, et.al., op. cit.). The specific choice of algorithmic technique depends upon the inspector's assessment of the general nature of the identified region.

Although we do not doubt that a sufficiently large and well trained neural system of the LILARTI variety could learn to perform the complete identification and evaluation process at any desired level of competence, we are uncertain that the required computing machinery could be acquired for an acceptable fraction of the GNP. It appears to us that a more efficacious use of the LILARTI methodology would be in emulation, not of the complete analysis process, but of the non-algorithmic process by which the human (given sufficient experience) identifies regions of potential interest. Once identified, these regions would be subjected to thorough evaluation by the algorithmic technique selected as most appropriate by the neural network. A LILARTI system large enough to perform the identification process with very high accuracy can be evaluated rapidly in suitably enhanced PC environments. In the same computing environment, the multiple-property method of Deeds and Dodd can be executed at essentially "real-time" speeds.

We suggest, then, the linking in one system of a suitably prepared LILARTI network and a version of the multiple-property code. The network would be trained in a wide variety of situations in which the training goal would be, not (as it is now) the full evaluation of a set of eddy-current images, but the correct identification of regions for which thorough evaluations were required. An additional training goal would be the selection of the set of multi-property program parameters best suited to the identified anomaly. Data relevant to each identified region would be submitted in turn to the multiple-property code for final assessment. It is our 
contention that the overall performance potential for a hybrid system of this sort far exceeds that of the best trained human/program combination.

As applications we should also consider the more demanding aspects of NDE for which the image oriented characteristics of the LILARTI system would prove particularly advantageous. As we have earlier noted, the eddy-current signals are presented to the network as they are to the human in an image format but qualify as images only in the most limited sense. The presentation of data in image form is largely a convenience for the human operator. Information is encoded entirely in the path traced in the complex plane as a function of time. There are no grey-scale data in either the "raw" patterns or in the line-crossing signal representations thereof. This should not be taken as suggesting that the LILARTI scheme is incapable of dealing with grey-scale data. Indeed, recent attempts at reducing the number of retinal pixels required for the eddy-current studies still further have led to a scheme in which the three line-crossing images are compressed into one in which pixel output value represents line-crossing "order". System performance in the context of these synthetic grey-scale data appears at least as good as that of systems employing the three separate binary sub-images.

It is natural, therefore, to consider turning our attention to the automated description of flaw indications in an NDE application for which the term "image" has a more conventional meaning. Such an application is non-destructive ultrasonic flaw assessment. Here, grey-scale data are everything. Moreover, the images are generally larger than those characteristic of the eddy-current problem and their storage as well as their processing become issues of importance. It is in this connection that our earliest vision-oriented work with the LILARTI systems would prove invaluable. As a part of that work, we developed a collection of "neural wirings" capable of preprocessing images in such a manner as to extract what may be termed "visual primitives" from presented scenes. Only those image regions incorporating the visual primitives in certain specified ways were subjected to subsequent neural processing. The resulting reduction in processing time and required neural storage space was dramatic. Since these "wirings" were developed we have explored mechanisms by which to force a LILARTI network of the newer type to codify, in the context of a given (albeit much simpler) environment, those wirings which consistently served to identify regions analogous to the noted visual primitives. One of the principal tasks of any ultrasonic image studies would be the incorporation of the codification scheme in a LILARTI network specifically constructed for ultrasonic image characterization. Once trained with a representative set of ultrasonic images, such a system would be evaluated in terms of its capacity for identifying those regions of an ultrasonic image where flaws of various trained-for types appeared.

A somewhat simpler image-oriented application for LILARTI networks can be found in the area of optical surface testing. A concern central to the production of high quality reflecting optical elements is the determination of the extents and natures of surface deviations from an "ideal" or intended geometry. Although there now exist sophisticated laser-based interferometric systems capable of making such determinations with very high accuracy and precision, these are expensive and not generally suited for application in routine optical shop work. For applications such as these (and for more complex ones as well) one of the oldest optical measuring schemes, the Foucault test, remains, when applied by a skilled technician, one of the most effective. Because its effectiveness does depend so heavily upon the interpretative skills of the technician, it is, in these modern and instrument-oriented times, less frequently employed than it should be. By the same token, it is this same dependence upon 
an acquired human skill which makes the testing method such a natural candidate for the application of artificial neural network methods. The product of a successful integration of the Foucault measuring scheme with the pattern recognition capabilities of a properly prepared neural network could be a very effective and robust system for analysis of errors in mirror figures.

In the first of the following paragraphs we describe very briefly the classical Foucault test and something of the method of interpreting results therefrom. In the next three, we discuss the incorporation of the fundamental ideas in a practicable neural network-based testing system.

The Foucault test as it is classically applied to a reflecting mirror requires examination of a sequence of shadows reflected by the mirror and cast by a knife-edge co-located with a source of illumination and the observer's eye. Careful, but easily made, measurements of the position of the knife-edge as it is moved along the mirror axis (in general, from one side of the center of curvature of a "best-fit" sphere to the other) coupled with equally careful assessment of the distribution of illumination in the shadow permit an experienced technician to determine to very high accuracy the deviations of the mirror surface from some desired configuration. Even in the hands of a novice, such distortions as turned-down edge, depressed center, radial and axial asymmetry, and the like are readily identified. With longer experience, errors of tenths of a wavelength and less can be readily quantified.

A system capable of demonstrating the capabilities of a neural network in the context of Foucault-based mirror surface assessment must include seven principal elements. Aside from the obvious requirement of a mirror and its means of mounting, there must be a lightsource and knife-edge assembly and a mechanism for moving it in a precisely controlled manner along the mirror axis. Associated with the knife-edge assembly must be some form of detector array at whose outputs will appear a digitized representation of the distribution of illumination in the knife-edge shadow. From this detector, there must be some means of communicating the signals in a properly ordered manner to a computing system for presentation to the inputs of the neural network embodied therein. Because the shadow variations on which the Foucault test depends are generally of low and subtle contrast, some form of contrastenhancing preprocessing is almost certain to be required prior to presentation of signals to the neural network inputs.

A demonstration apparatus capable of illustrating the important points can easily be assembled from readily available modular optical components. Because spatial resolution is of relatively less importance than grey scale accuracy, only very simple video components should be required for the signal acquisition process. The principal limiting factor in a demonstration system is likely to be presented by the computing system in which the neural network must be represented. However, since great speed is of little importance for proof-of-principle purposes, a 486/860 combination should provide more than adequate processing power.

One of the advantages of the proposed approach is that system training can be performed in the context of synthetically generated Foucault patterns. This is indeed fortunate as it would be impracticable to base training on the preparation of a perfect test mirror of every desired class, diameter, and focal length. It is now straightforward to generate algorithmically the sequence of shadow patterns which would obtain for any combination of errors in a reflecting element of any specified form. For demonstration purposes, one would select the simplest 
aspheric, the parabola, and train the network in the context of generated shadow patterns for the sorts of errors generally encouniered. The testing aspect of the demonstration would then be performed with perhaps a dozen commercially obtained mirrors of intentionally disparate quality. Finally, results of the network's assessments of these mirrors would be compared with those of an experienced technician and with results obtained by automated interferometric measurement.

Essential to these or almost any other generalization of our work is the overcoming of limitations inherent in the computing equipment to which we have so far had access (16 MByte 486-PC with Weitek co-processor or 386-PC and Iniel 1860). One of the disadvantages of the LILARTI scheme derives from the relatively large number of neural and connection parameters which must be stored and manipulated during network processing. Thus, effecting a fair evaluation of the ultimate potential of the method, particularly in the context of more data-intensive NDE applications like ultrasonic imaging and $x$-radiography, will require a computing system with considerably more processing speed and much enlarged memory. Such a system can now be assembled in a PC environment from off-the-shelf plug-in modules for no more than forty-five thousand dollars.

We conclude this section with some suggestions for those more interested in the general properties of neural networks than in their applications. We begin with a notion which has long intrigued us, the "question answering" neural network. By this term we intend to suggest a neural system which, instead of responding to a single set of stimuli with some sort of bytraining associated descriptive parameters, accepts two sets of stimuli (one of which might define an image) and responds in a manner predicated on the relationships obtaining between the two sets. The (almost) tri-laterally symmetric wiring depicted in Figure 11 (and in more normal LILARTI format in Figure 12) suggests the central ideas. In these figures (which represent only the simplest variation on the general theme!) the parenthesized lists denote neural sheet positions in a LILARTI configuration and the adjacent "crossed" number pairs the sheet sizes. The inter- and intra-sheet connectivity schemes are assumed to be of the simple forms described in the material of the appendices on LILARTI data file construction.

In the illustrated example, each sensory input drives regions of the other two senses through trainable connections and drives, by 10 connections, a collateral top level sheet which is trainably driven by the other senses. If one of the sensory inputs is a retina, and if the other two are respectively a "parameter" input and a "value" input, the system will learn, with training, how to associate parameters with values which are appropriate to those parameters in the context of various presented images. Following training, presentation of an unfamiliar image and a parameter type for which the system has been trained (in effect, a "question") will produce at the output of the "value" sense an estimate of the degree to which the specified parameter is represented in the image. Similarly, if an image and a value are presented, the system should be able to suggest the parameter with which the given value is most nearly consistent. Last, given a parameter and a value, the system should reconstruct at least those parts of an image for which the parameter/value combination has meaning.

Although we would certainly not argue that the just described system is biologically accurate, it does extend the notion of cognitive complementarity which we have found so engaging beyond the two senses to which we have so far applied it. Perhaps most appealing to us is the possibility that such a system could capture something of the notion implicit in that most 
ORNL-DWG 92-11942

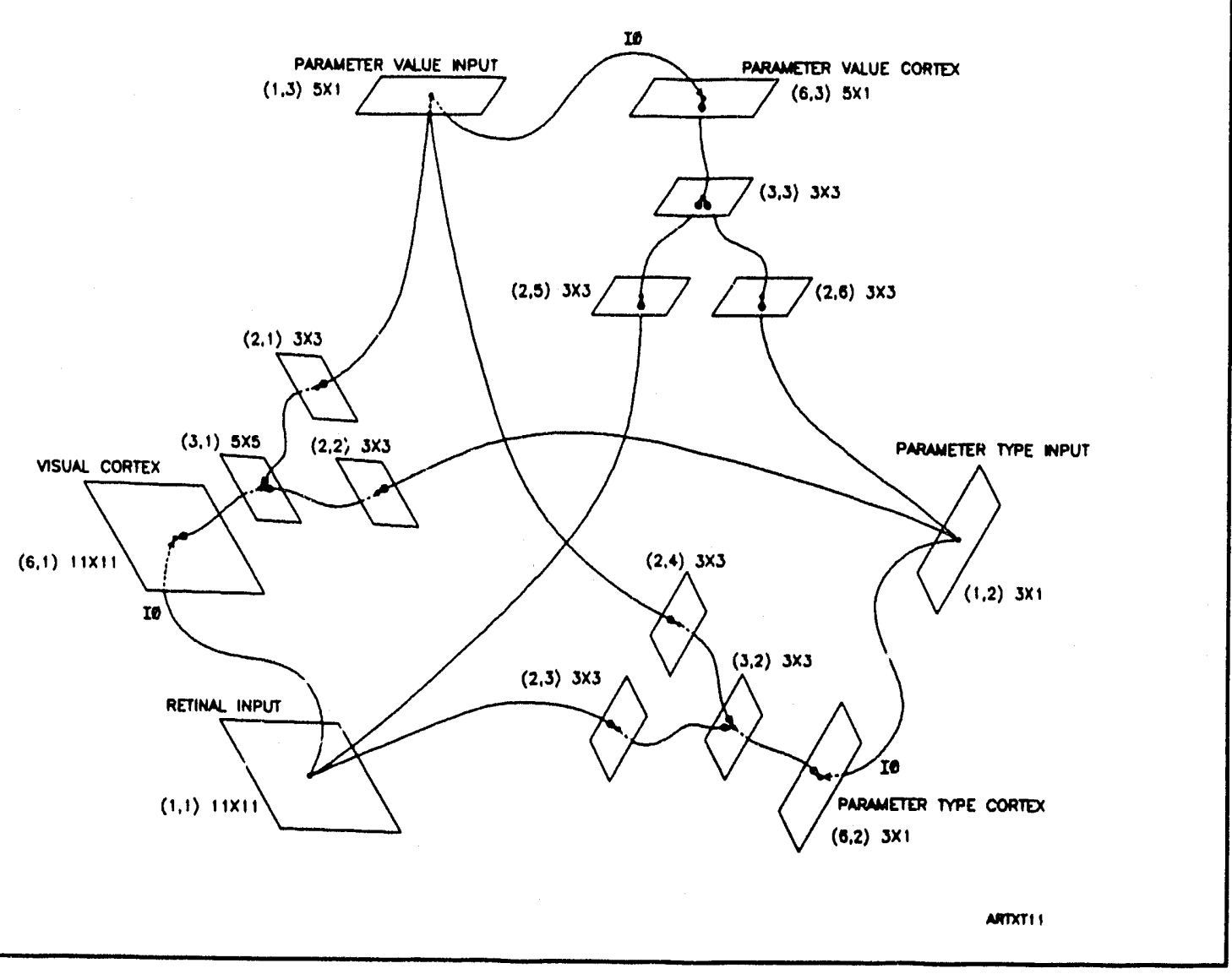

Fig. 11. Tri-lateral question answering network

elusive of terms, "concept". If, for instance, the system had been trained in the context of a very large number of image/parameter/value combinations, it is reasonable to suppose, as indeed we suggested above, that, given an unfamiliar image and a trained-for parameter (e.g., "size") as inputs, the system would be able to respond with a value consistent with the two inputs. If such behaviour were observed, it might be argued that the system had come to "understand" the concept of size. More specifically, if, for presented, previously untrained-for, images of a house, an airplane, and a boat, the system returned "large" for the value of "size" and if, for chair, table, and desk, it returned "medium", it would be hard to argue that some abstracting process were not at work in the network. We contend that, given suitably simplified "images" and a sufficiently restricted set of parameter types and values, a system capable of exhibiting the suggested behaviour could be processed in PC computing environments.

A direction which we can no more than mention in passing involves neural network extensions of our work with artificially intelligent systems for organic chemical synthesis ${ }^{13}$. The LISP. based non-neural CANONMAN systems of Allen and Schell have proved very successful at learning by experience something of the flavour of analogical reasoning as it is applied by chemical synthesists to the processes of developing synthetic pathways for user-specified target molecules. What it can not do is codify the broad principles which are inherent in the 


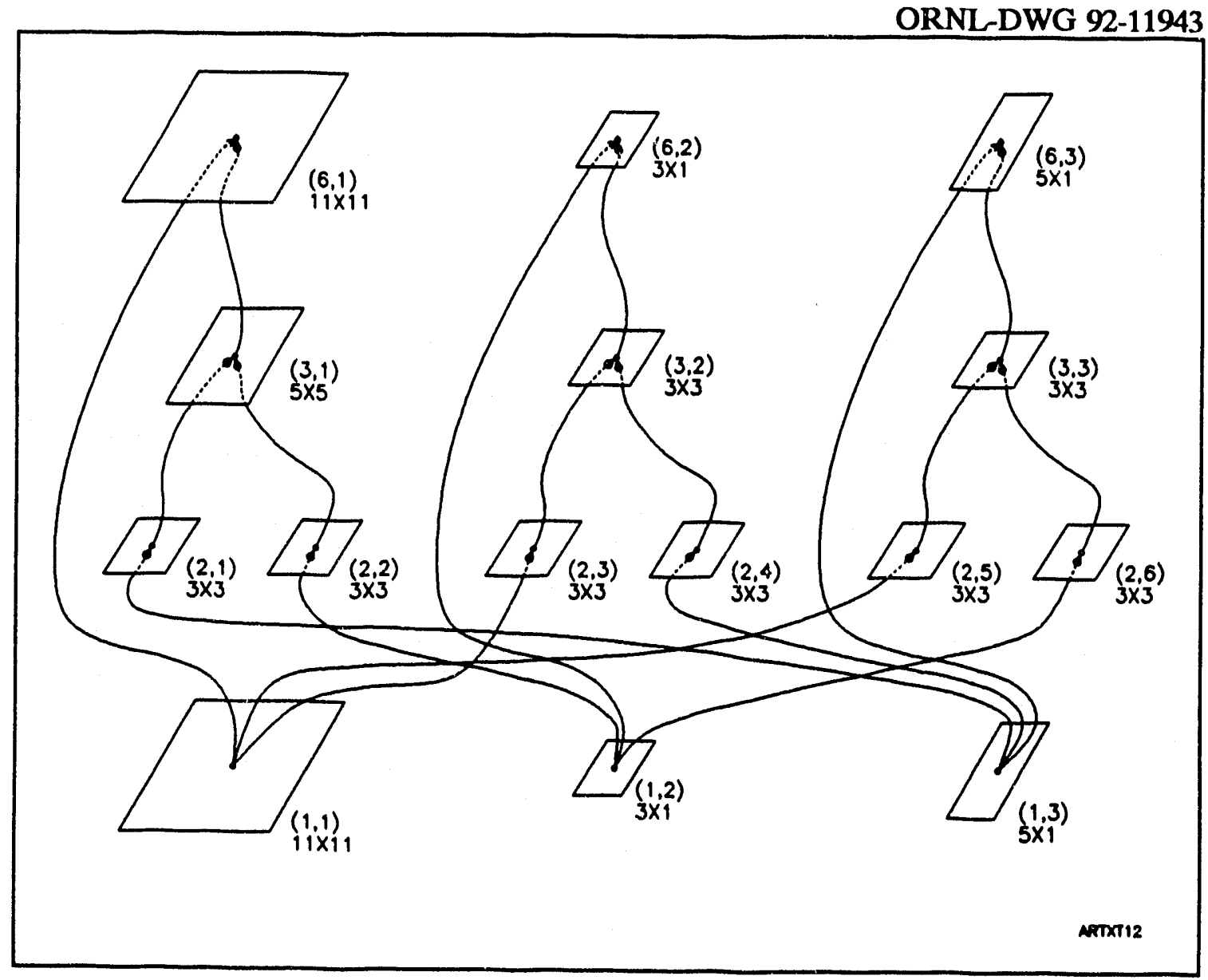

Fig. 12. Normal form representation of question answering network

methods it learns to apply. Such a codification process lies within the natural purview of neural networks. We are confident that training a network in the context of the nodal information which comprises the canonical tree representations of the CANONMAN system would lead to a system capable of applying its knowledge far more effectively than does the present system.

With considerable trepidation (because of the immensity of the associated tasks, our inevitable inadequacies relative to them, and the impossibility of presenting in condensed form a description which does not trivialize the associated ideas), we conclude with what, here, can be no more than the merest suggestions about two topics to which we have given considerable thought and to which we hope ultimately to be able to devote equally considerable research effort. We refer to the questions of natural language acquisition, and the incorporation of training in the context of biological needs. Indeed, in our view, these are inseparably linked notions and should not be viewed in mutual isolation. The relation between these two, which may not at first appear obvious, is an inherent dependence of the associated behaviours on time. 
That natural language development is inextricably linked to dynamical processes is clear. If it appears less so that the same dependence obtains for training of such nature as is required to improve response to biological needs (whether for the alleviation of hunger, protection from the elements, or any of many others), it is at least in part because the time scales for the associated processes are very different. Another disparity to note is that, whatever the training methods may be for true biological systems, it is probable that changes in "need" (e.g., the onset and alleviation of hunger) rather than the need itself are more suited to the generation of signals capable of directing the learning process. The important point is that variations in time are important to both of these seemingly disparate activities.

We do not mean to suggest that the neural processes of training to accommodate needs and those associated with language acquisition are equivalent. In connection with the former, very simple neural systems can detect the temporal variations in the multitude of signals involved in the simplest biological function, and can, by their outputs, support training both for those activities which reduce some need and training against those which increase it. It is, of course, not enough merely to detect changes in need. So that there will be something to learn, a mechanism must be present by which the "organism" can experiment with actions (both external and internal) which may have some influence on the level of the need. Further, because variations in need may not be contemporaneous with those actions which ultimately prove capable of affecting the need, something resembling a short-term associative memory must be provided for in which to retain the relationships between the various attempted "solutions" and variations in the magnitude of the need. If the states of this short-term memory are, over time, employed by the organism as training stimuli for itself, only those attempted solutions which have proved consistently efficacious in need reduction (whatever the time delay) will lead to the establishment of trained states representing a new behaviour pattern.

Whereas a collection of suitably modified and interconnected LILARTI (or almost any other) networks could accommodate each of the characteristics suggested above in the context of some "toy world", no such congruence would be found between the requirements of natural language acquisition, at least as we choose to define that activity, and the capabilities of the present LILARTI. For us, the acquisition of language is more than the development of the capacities for recognizing and uttering strings of syllables in some trained-for order. Indeed, very simple networks can be arranged to participate in either activity. We choose instead to define language acquisition as a process whereby an organism (natural or "artificial") learns, by interacting with its environment and others of its "species", not only a consistent set of symbols for the elements and operations of its environment, but a relational grammar by which it can manipulate that environment in response to its own needs.

It is necessary only to enumerate the processes associated with the simplified representation of language acquisition just noted to gain some appreciation of the magnitude of the problem. Even restricted to the most limited "toy world", a language acquiring organism would require sensory input of sufficient quantity and quality to permit the development of unique internal descriptions of the elements and operations of its environment and of relationships among them. Part of this input may be "visual", but, if the organism is to learn language by interaction, part must be the linguistic output of an external "entity" which takes the role of an interacting member of the organism's species. This entity must be able to "point" and to associate linguistically correct cues with at least the noun and verb aspects of its actions. 
Although psychologists may contest the issue, we contend, further, that, without a anpacity for influencing its environment, any organism would develop language in more than an ...itative way (the utility of which we do not discount) only slowly, if at all. This influence should, we believe, be exerted linguistically. As already suggested, there must be something associated with the learning organism representing an experienced need, a need ameliorated only by success at the utilization of its evolving language in manipulating its environment in increasingly complex ways.

If, in the term language acquisition, we include (and we do) the development of a capacity for parsing non-trivial syllable strings in terms of some consistent and evolving structural representation scheme capable of capturing the perceived state of the environment and, further, a capacity for constructing non-trivial syllable strings in the context of this representation which express operations to be performed on the environment, the problem (and this sentence) approaches intractability! Certainly, it cannot be addressed in the context of LILARTI systems of the present sort for these are, despite the dynamic "dithering" process by which training occurs, essentially static systems. Other difficulties aside, such systems could not (and this point is important) directly respond to or generate the highly time-dependent syllable strings characteristic of anything one might hope to refer to as "speech". Although we can see LILARTI solutions for every other aspect of language acquisition, we must contrive something entirely different for at least this one. What presently seems most likely to us is a system in which training leads to the production, not of static regions in neural state space each perhaps representing some word, but dynamic regions in which stable, quasi-periodic "orbits" exist. We imagine the trajectories of these orbits as providing the mechanism for the production of temporal sequences of all sorts, including those required for syllable recognition and production. Similarly, it must be arranged that the proper syllabic order be associated, by training, with each newly acquired word. Most current networks could manage this feat. What is not so easily accomplished is the extraction of "meaning" from ordered word strings or, inversely, the production of ordered word strings from meaning.

It is at this juncture that the widely suggested notions of an inherent "grammar-oriented" neural structure begin to exhibit their appeal. Indeed, the neural network language acquisition model which we are (slowly) beginning to explore captures in a very crude way some of the essential ideas. Omitting all reference to the temporal aspects which we consider so important, we imagine a primitive "organism" comprising six essential regions. There are sensors by which to assess the environment and effectors by which to produce some modification thereof. Three separate network regions connected in sequence link the sensors to the effectors. Each of these includes trainable and (more primitive) non-trainable subregions. We arrange for the first region to perform the simplest of "scene" parsings. The second can, we suppose, in its initially untrained state recognize some simple relations among at least some of the parsed elements. The last, again in its initially untrained state, recognizes some combination of the relations and produces (almost certainly incoherent) outputs to the effectors. In the crudest senses of the terms, the three network regions may be thought of as representing primitive "nouns", "prepositions", and "verbs".

The outputs from the "verb" region drive, in addition to the effectors, the inputs of the sixth region whose output is associated with the sensors. We think of this sixth region as the locus of a crude internal "world model" which the organism builds with experience (from sensory inputs and its own responses to them) and by which it is increasingly able to detect disparities 
between expected "actions" in its world and the actions actually sensed. The disparities are used both as cues to the correction of the internal world model and as initiators of the training mechanisms by which the system refines its abilities to parse scenes, extract relationships, and direct actions.

Even with the inclusion of the temporal aspects which we think we can arrange for ${ }^{14}$, the model briefly sketched here could not be expected to learn with anything approaching linguistic richness. However, it should be capable of learning about its environment (if that environment is of sufficient simplicity) in terms of an acquired language which would include at least crude nouns, prepositions, and verbs. With the incorporation of something like the trilateral system noted earlier, adjectives could be included in this list. What appears to us to be unique about this system is that the process of language acquisition, because it is interactive, leads to a facility, not just for recognition, but for directed, goal oriented, use. 


\section{ACKNOWLEDGMENTS}

We would be remiss were we to fail formally to thank all those whose efforts and good will contributed so vitally to our work. In approximate order of their initial contributions, we happily acknowledge the following: Fred Mynatt and Bill Eads who, as successive directors of the I\&C Division, looked with favour on the efforts of three non-engineers; Charles Mossman and Daniel McDonald who, as successive heads of the MACES section of the I\&C Division, supported JDA when funding from outside sources was unobtainable; the ORNL Seed Money Committee for one year's support; Randy Pate, as gifted a young physicist as we have encountered, for preparation of the training data set; L. D. Chitwood for preparing, always on short notice and with boundless good spirits, the figures: and Chris Bhagat of Wright Patterson Air Force Base for continuing support; and Donna Balltrip for final manuscript preparation. 


\title{
APPENDICES
}

\author{
Appendix A1 \\ Instructions for Using LILARTI
}

The material of this appendix is divided into two major sections. In the first, we describe how to operate the LILARTI system using source code and data files available from the authors. Found here will be suggestions which should be useful to the beginning user. In the second, we provide some general instructions for preparing data files capable of directing the generation of networks of the user's design. We note that the source code was written in NDP Fortran and incorporates a few NDP-specific functions which may not be supported in other Fortran versions. Among these are TIMEDATE, CLEAR_TEXT, and SET_VIDEO_MODE. References to these functions can be eliminated if substitutes are not available. More problematical is the INTEGER*2 function INKEY\$ which returns an INTEGER*2 value representing the contents of the keyboard buffer at the moment the function is evaluated. In the LILARTI code, INKEY\$ serves primarily as a mechanism for escaping from long processes or multiply traversed loops in a manner requiring no action from the user when escape is not desired (and encountered READ, for instance, would require at least a carriage return before processing could continue). A minimally satisfactory solution, if no suitable mechanism can be found for duplicating the effects of the INKEY\$ function, is the removal of all references to it. We strongly recommend the writing of a few lines of assembly code for incorporation of the necessary operations in the manner supported by the user's Fortran environment.

\section{A1.1 \\ Running LILARTI}

\section{A1.1a System Utilities}

As an introduction to system operation, we reproduce here the essential elements of the HELP list and provide such descriptive material as appears necessary. For each listed operation, the expected user response is noted. As a nearly general rule, short-hand versions of these, often comprising the first letter (when this is unambiguous) or letters (when it is not), will invoke the desired function. When in doubt, consult the code.

Run Network (RUN):

This command invokes, upon a further selection by the user, the simplest operational modes of the LILARTI system. These modes are three in number of which the last, the so-called "EXHIBIT" mode, has, in the present LILARTI context, very little meaning. If the subsequent response "LEAR! is given, the user will be asked a few questions concerning training image number, number of training attempts, and print and plot requirements. If "NONLEARN" is the response, the system wiii again ask for an image and for print and plot directives and will further inquire as to whether or not the user wishes to perturb the image before processing. A "YES" here invokes the "PERTURB" utility and leads to more questions. When processing is complete, the states of the top level sheets 
will (assuming a typical LILARTI wiring) indicate the overall system response to the presented image.

Train Network (TRAIN):

Invoked by this command is a conventional brute-force training method in the context of which the network is repetitively exposed to the members of a training set (presently some or all of either the test images or the NDE images). The user can manually select the specific members of the training set from the complete image set or he can specify a fraction of the complete image set for use as the training set and leave it to the program to pick the required number of elements at random. Upon gathering a few more training instructions, execution begins and continues until one or another of several well-defined end conditions is satisfied. In contradistinction to the operation of the next-described TUTOR mode of training, all training images are presented to the network during each training pass. Only the order of presentation (determined by a simple random shuffle process) changes from pass to pass.

Tutor Network (TUTOR):

As suggested previously, the Tutor training mode represents an early attempt to incorporate some of the more important characteristics of training methods known to be effective in humans. In its earliest realizations, its functions modeled more or less crudely the general sequence of operations already described. Although present versions of the code still support the earliest form of TUTOR mode training, this utility has come to be more nearly a variation on the TRAINING mode. Only if the user hand-selects and appropriately distributes the members of the training set will conventional TUTOR mode training obtain. If the system is allowed to select training set members, they will be distributed at random over the groups originally intended for the representation of closely similar images. Thus, each group sparsely represents the whole. By analogy with TRAINING mode operation, the order of presentation of the elements of a training group is determined by a random shuffle before each training pass.

\section{Test Tutor (TESTTUT):}

This function is usually invoked when a TRAIN or TUTOR training session is complete. The program tests the performance of the trained network on those elements of the full image set (whether NDE or TEST images) which have not been used for network training.

The remainder of the system functions may properly be viewed as utilities by which the user may explore various aspects of LILARTI system functioning. It will be clear to the reader that some of these were written for a specific and limited purpose (usually as a "debugging" aid) and are now of little use.

\section{Generate Nness Image (GENN):}

This function reflects the motivation for the original LILARTI studies, the genetically-based search for network geometries capable of emulating the process(es) by which certain humans learn to recognize and manipulate random 
dot patterns in a mathematical context. Thus, invoking GENN generates and loads onto the "retina" an "image" of $\mathrm{N}$ randomly distributed "on" pixels.

Generate Image (GENIMG):

The system response to this command depends upon the application selected by the user as execution began. If $T$, a selected element of the 81 member test image set will be generated and loaded onto the "retina". If $\mathbf{N}$, the program will retrieve a user-selected NDE image from storage and load it onto the "retina".

Display a Selected Image (DIMG):

This function provides the user the convenience of combining in one command the keystrokes required to generate and display a selected image. Here, as elsewhere, the selected image will be one from the set associated with the current application. Thus, it is not possible to display NDE images when in the TEST application nor can TEST images be accessed when in the NDE application.

Compare Images (COMPIMG):

This is one of the more useful functions as it permits the user to determine the "similarity" of two specified images. The user should be aware that similarity is defined here as a measure of more than the pixel-by-pixel equivalence of two images, although pixel-pair evaluation plays an initial role in the similarity computation. Instead, for each equivalent pixel, an inverse radius-weighted measure of "global" similarity is determined. It is the normalized sum of these which is returned as the "similarity". The intent here was to emphasize the effects of cluster similarity.

Perturb Image (PIM):

This function provides the user with the facility for manipulating generated images and is useful for, among other things, ascertaining the sensitivity of a trained network to various image perturbations. The presently supported perturbations are briefly commented on below.

Rotation $=$ Rotate retinal image by a user-specified angle (in degrees)

Translation $=$ Translate retinal image by user-specified numbers of pixels in "X" and "Y"

Scale Alteration = Change image scales in "X" and "Y" by user-specified fractions

Random Thinning = Remove (set in zero) at random a user-specified number of active retinal pixels

Addition of Amplitude Noise = Randomly perturb the retinal intensity distribution by a user-specified amount

Addition of Position Noise $=$ Randomly perturb the positions of the active retinal pixels by a user-specified maximum displacement amplitude 
Fire Selected Neuron (FIREN):

When given this response, the program asks the user for four neuron-locating coordinates (level, sheet, row and column, or $\mathrm{K}, \mathrm{N}, \mathrm{I}, \mathrm{J}$ ) and sets the output of the selected neuron to the value corresponding to the maximum output for neurons on the specified level and sheet.

Fire Selected Neural Sheet (FIRES):

Although this command, like the previous, results in forced neuron firing, here the effect is sheet-wide. When level and sheet coordinates are provided, all neurons on the specified sheet are examined. The outputs for those bearing non-zero input are set to the value corresponding to maximum output for neurons on the specified level and sheet.

List One Connection Weight (LSTCONNWT):

This utility permits the user to determine the characteristics of all connections linking a specified source neuron (at some KS,NS,IS,JS) with a specified target neuron (at some KT,NT,IT,JT).

List One Neuron (LSTNEURON):

Upon receiving this command, the program lists all state information for the neuron at a user-specified position.

List All Input Connections (LSTALLIN):

This is one of the most useful of the utilities as it permits listing with one command all the input connections to the neuron at a user-specified location. The listed parameters include, for each connection, the source neuron coordinates, the present connection strength, and the current value of the connection catalyst.

List All Output Connections (LSTALLOUT):

This utility lists all the output connections from the neuron at a user-specified location. The listed parame' ers are of the type and form noted above. The user should be aware that some processing time is required for execution since the connections are being viewed from the "wrong end".

List All Active Sources (LSTAS):

This code of this utility is functionally similar to that of the LSTALLIN routine previously noted. However, only those connections for which the source neuron output value exceeds some system-set value are listed. Pre-synaptic and Postsynaptic signals, target-terminal indication, and the associated connection weights are included in the presented listings.

List Level Parameters (LSTLEVELPARM):

This utility, and the next three, permit the user to examine the contents of the system defining file (e.g., LILARTYI.DAT) from which the current network structure was derived. The file is divided, as the utility command names suggest, into four parts, LEVELPARM, CORTPARM, CONNPARM, and SIGPARM. 
List Cortical Parameters (LSTCORTPARM):

See "List Level Parameters".

List Connection Parameters (LSTCONNPARM):

See "List Level Parameters".

List Sigmoid Parameters (LSTSIGPARM):

See "List Level Parameters".

List System Parameters (LSTSYSPARM):

At this response, the program lists some of the principal system-defining parameters and statistics.

List Defined Target Sheets (LSTKNTDEF):

This utility lists for each level/sheet its status as a defined target sheet, i.e., its bearing of (1) or failure to bear (0) input connections.

List Defined Source Sheets (LSTKNSDEF):

This utility lists for each level/sheet its status as a defined source sheet, i.e., its bearing of (1) or failure to bear ( 0$)$ output connections.

List Trainable Sheets (LSTKNLRN):

This utility lists for each level/sheet its status as a trainably connected target sheet, i.e., its bearing of (1) or failure to bear ( 0$)$ trainable input connections.

List Random Connection Sheets (LSTKNRAND):

This utility lists for each level/sheet its status as a randomly connected target sheet, i.e., its bearing of (1) or failure to bear ( 0 ) connections generated by the random connection generation process.

List RELAX Copy Sheets (LISTKNCOPY):

Intended only for debugging purposes, this utility lists for each level/sheet its status in the context of the dithered-state processing. Only those neural sheets denoted by " 1 " in the listing are copied into temporary array locations for purposes of the relaxation operation.

List [KSNSISJS] starting locations (LSTKNSTART):

This is another of the "debugging utilities" and permits listing the location in the connectivity array at which input connections to the user-specified target neuron begin. Note that connections form contiguous blocks in the KSNSISJS array, each block corresponding to all the inputs to one neuron.

List Weight Alteration Statistics (LSTKNSTAT):

At this response, the program lists (if they have been collected during system training) such global training statistics as the number of connection alterations involving each pair of trainably connected sheets, the total of the associated weight alterations, and the like. Since training proceeds somewhat faster if the 
user suppresses the collection of training statistics, this statistics collection feature should be used only when the results are likely to be of interest.

List Total Level/Sheet Fanin Values (LSTFAN):

This command results in the listing of typical fanin values for neurons on a specified level/sheet or on all level/sheet pairs. Note that the "one or all" listing feature is common to most of the system listing utilities.

List Level/Sheet Activity (LSTACT):

At this response the program prints the various measures of activity and the states of certain flags and measures for the specified level/sheet or for all level/sheet pairs. These include the Total Activity (the sum of all neural outputs), the Specific Activity (the Total Activity divided by the number of active neurons), the Current Sheet Stability and the Old Sheet Stability, the Sensor Input Flag (an indicator of the presence of External Input signals), the Inter-Neural External Input Flag (an indicator of the presence of Inter-Neural External, or I0, Input signals), the Pending Dither value (an indicator of the number of neurons yet to undergo the "dither" process), and the Pending Train value (an indicator of the number of neurons yet to undergo the training process).

List Tutor Results (LSTTUT):

This command is useful following training and forces the listing of some of the more relevant training statistics.

Print Image (LSTMMG):

Differing in function from the Display utility described below, the code of this utility simply prints output values for the neurons comprising the retina and serves principally as a debugging tool.

\section{Print Sigmoid (PRNTSIG):}

The code of this function and the next two comprise two more debugging utilities. The response here is to list the values appearing in the Sigmoid array for a user specified level/sheet.

Print Sigmoid Inverse (PRNTSIGINV):

This utility performs for the Inverse Sigmoid array the function performed by the previously noted utility for the Sigmoid array.

Print Cortical Sheet (PRNTCORT):

A generalized version of the Print Image utility, this function lists neuron output values for any user-specified level/sheet.

Invoke Adjustment Utility (ADJ):

Of the three functions supported here (Connection Weight, Connection Catalyst, and Screen Plot Parameter Adjustment), only the last has any real utility in the present LILARTI. The first two permit user adjustment of the indicated connection parameters for a connection linking two user-specified neurons. The 
ambiguity associated with the possibility that more than one connection can link a neuron pair is not resolved.

The Screen Plot Parameter Adjustment, on the other hand, can be of considerable value as it permits selection of the neural sheets which will be displayed during execution and the types of plots which will be produced. The plot types presently supported are listed below:

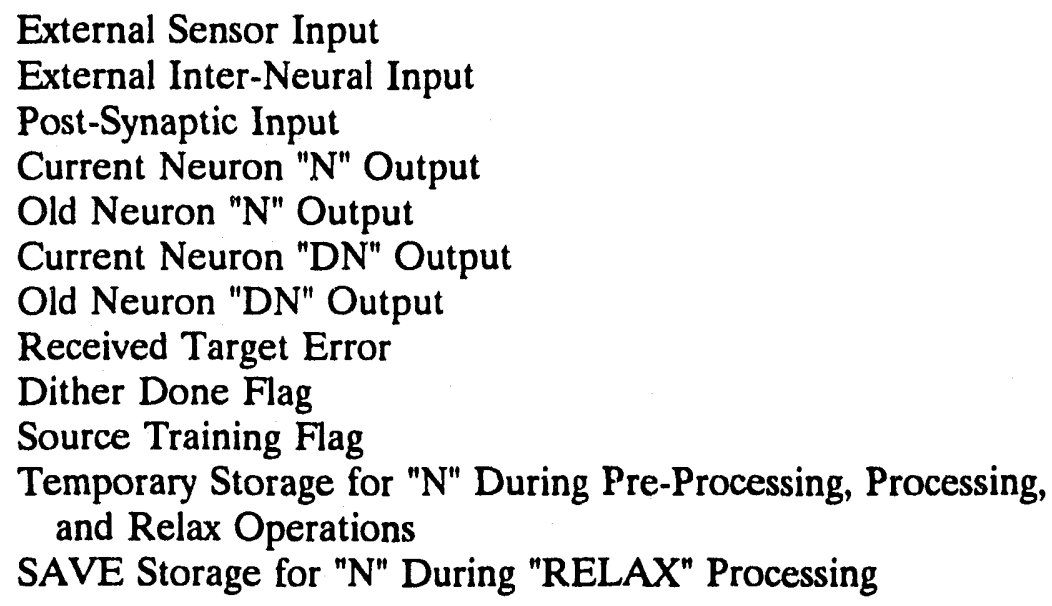

Invoke User Display Utility (DISP):

This, although invocable only when neural execution is not in process, is surely the most useful of all the utilities as it permits the user to examine graphically and (although in only modest detail) most of the important characteristics of the various neural sheets. The "SS" (single-sheet) option selectable after the DISP response is the most frequently applicable mode. However, where the difference in some parameter (or parameters) between two sheets has meaning, these differences can be displayed by choosing the "SD" (sheet-difference) option. The presently supported displayable parameters are noted below:

External Sensor Input

External Inter-Neural Input

Stored Post-Synaptic Input

Computed Post-Synaptic Input

Current Neuron "N" Output

Old Neuron "N" Output

Current Neuron "DN" Output

Old Neuron "DN" Output

Received Target Error

Dither Done Flag

Source Training Flag

Temporary Process

RELAX "Save N"

Differential "N" Output Plot

Differential "N" $" \mathrm{DN}$ " Output Plot

Pre-Synaptic Neuron Input 
Total Neuron Input

Weights

Catalysts

Save Total Connectivity on File (SAVECONN):

At this command the program saves (on a file of the user's choice) the current system wiring. As noted earlier, the current status of this and the next utility is uncertain.

Read Total Connectivity File (READCONN):

At this command the program reads (from a file of the user's choice) a complete system wiring.

\section{Show All System Parameters (SHOWSYS):}

This provides an unnecessarily verbose listing of the connection defining and Sigmoid defining parameters.

\section{Reset Catalyst Values (RESETCAT):}

At this command, the catalysts for all system connections for which non-zero catalyst values are allowed are set to the maximum permitted value (in the current LILARTI, 32000).

Restore System (RESTORE):

At this command, the program restarts at the Application (TEST Image or NDE) $>$ prompt. All traces of any prior system are erased.

Clear Cortical Sheet (CLEAR):

Originally intended principally as a debugging tool, the utility invoked at this command has proved more generally applicable. When accessed, it permits the user to reset most of the neural parameters for a specified sheet (or for specified sheets).

Write System Data (WSD):

This utility writes the elements of the more important system arrays to a file. Intended as a debugging utility the function now serves no useful purpose and could be removed.

\section{Print Average Processing Times (PRNTIME):}

At this response the system prints the processing times for the most recently performed operation(s).

Although it is not possible, with so brief a survey, to describe system operation in any substantive detail, the serious user, given the material here, should be able to develop real facility in a matter of hours. We would suggest beginning with the simplest of the training modes (RUN followed by LEARN, etc.) so as to acquire an appreciation for the behaviour of the system as it learns one image. Response to training can be studied by using RUN followed by NONLEARN. Use of the plotting and printing utilities will prove useful here (although we warn that the VERBOSE printing mode can severely compromise system speed 
and the user's patience). Operation in the TRAIN mode should be explored next (having first used RESTORE to restart the system). Five or six images will be learned in only a few training cycles at two or three training attempts per cycle. When these images are fully trained for, the user should examine system responses both to these and to as yet unlearned images using RUN followed by NONLEARN, as before.

\section{A1.1b System Execution}

Once an executable image is prepared, system operation is straightforward. We illustrate the process with a listing (throughout which we have dispersed comments of, we trust, a helpful nature) of the interaction between the program and a user. For convenience, the listing appears in bold type, the comments in normal type. Note that the code is arranged to require, almost universally, upper case responses from the user and that the expected forms (for each of which several abbreviations are accepted) of these responses are usually provided as cues at the program-generated prompts. Operation begins when the user types RUN LILARTI and continues as indicated below.

The program first determines the application which the user intends to invoke. TEST (or T) refers to system operation in the context of a set of 81 program-generated test images, NDE (or $\mathrm{N}$ ) to operation involving eddy-current images stored in the file NDEDATA.DAT. We shall illustrate only the TEST mode. Recall that a list of system utilities and the associated prompts is available upon typing HELP at the NEURONS> prompt.

\section{Application (TEST Image or NDE) $>$ T}

The nature of the network connection generation process is next ascertained. Setup from a parameter file leads to network construction in terms of the so-called "genetic" elements referred to in earlier sections and discussed in more detail in Appendix A1.2 and the several sections of Appendix A2. Setup from a Connectivity File involves only the reloading of a previously generated connectivity distribution. Indeed, for the applications of recent interest to the authors, a connectivity file is so rarely required that the present state of the supporting code is uncertain. We illustrate only the Parameter File-Based setup mode.

Choose Setup Mode:

(1) Parameter File Based

(2) Connectivity File Based

\section{SETUP> 1}

Respond With Connection Parameter Filename. New Filename ("NEW") or Default Filename (DEF") > D Reading System Defining File: LILARTYL.DAT

As the parameter file is read various useful system parameters are developed and printed for the user:

Maximum Level Number (KCORTMAX) $=6$

Maximum Sheet Number (NCORTMAX) $=12$ 
Depth of Deepest Sheet (ICORTMAX) $=11$

Width of Widest Sheet (JCORTMAX) $=11$

Retinal Size (at $K / N=1 / 1)=11$ by 11

Keyboard Size (at $K / N=1 / 10)=3$ by 5

Maximum Connection Class Number (NCONNCLASS) $=2$

Initial Maximum Fanin Value (MAXCONN) $=58$

Largest Number of Sigmoid Values (NSIGMAX) $=256$

Data Acquisition Complete

Having acquired all the system defining data, the program next determines the maximum storage space which will be required for connectivity representation. The figure printed for the user represents the total number of system connections.

\section{Connectivity Array Length: 1179}

Before beginning network construction, several checks are made to ensure that the usersupplied data can lead to the successful generation of a physically plausible network. The user should be aware that the authors were at pains to detect the sorts of potential errors with which they frequently afflicted themselves but cannot guarantee that all possible gaffes are provided for.

\section{Checking Inter- and Intra-Cortical Connectivity Parameters}

Now, the principal connection-defining parameters are listed for the user. Note that the listing process can be disabled at any point by striking " $Q$ " (and re-enabled by striking " $Q$ " again). This same toggle mechanism is incorporated in most of the listing utilities.

\section{Initial Sheet-to-Sheet Connection Distribution:}

(1) $\mathrm{KS}=1 \mathrm{NS}=10 \mathrm{NC}=1 \mathrm{KT}=2 \mathrm{NT}=10 \mathrm{Fan}=1 \mathrm{IR}=0 \mathrm{JR}=0 \mathrm{IL}=0 \mathrm{JL}=0 \mathrm{PI}=0 \mathrm{PJ}=0$

(2) $\mathrm{KS}=1 \mathrm{NS}=1 \mathrm{NC}=1 \mathrm{KT}=4 \mathrm{NT}=1 \quad \mathrm{Fan}=49 \mathrm{IR}=3 \mathrm{JR}=3 \mathrm{IL}=1 \mathrm{JL}=1 \mathrm{PI}=0 \mathrm{PJ}=0$

(3) $\mathrm{KS}=4 \mathrm{NS}=1 \mathrm{NC}=1 \mathrm{KT}=4 \mathrm{NT}=1 \mathrm{Fan}=1 \mathrm{IR}=0 \mathrm{JR}=0 \mathrm{IL}=0 \mathrm{JL}=0 \mathrm{PI}=0 \mathrm{PJ}=0$

(4) $\mathrm{KS}=4 \mathrm{NS}=1 \mathrm{NC}=2 \mathrm{KT}=4 \mathrm{NT}=1 \quad \mathrm{Fan}=8 \mathrm{IR}=1 \mathrm{JR}=1 \mathrm{IL}=1 \mathrm{JL}=1 \mathrm{PI}=0 \mathrm{PJ}=0$

(5) $\mathrm{KS}=1 \mathrm{NS}=1 \mathrm{NC}=1 \mathrm{KT}=4 \mathrm{NT}=2 \quad \mathrm{Fan}=49 \mathrm{IR}=3 \mathrm{JR}=3 \mathrm{IL}=1 \mathrm{JL}=1 \mathrm{PI}=0 \mathrm{PJ}=0$

(6) $\mathrm{KS}=4 \mathrm{NS}=2 \mathrm{NC}=1 \mathrm{KT}=4 \mathrm{NT}=2 \mathrm{Fan}=1 \mathrm{IR}=0 \mathrm{JR}=0 \mathrm{IL}=0 \mathrm{JL}=0 \mathrm{PI}=0 \mathrm{PJ}=0$

(7) $\mathrm{KS}=4 \mathrm{NS}=2 \mathrm{NC}=2 \mathrm{KT}=4 \mathrm{NT}=2 \mathrm{Fan}=8 \mathrm{IR}=1 \mathrm{JR}=1 \mathrm{IL}=1 \mathrm{JL}=1 \mathrm{PI}=0 \mathrm{PJ}=0$

(8) $\mathrm{KS}=2 \mathrm{NS}=10 \mathrm{NC}=1 \mathrm{KT}=6 \mathrm{NT}=9 \mathrm{Fan}=1 \mathrm{IR}=0 \mathrm{JR}=0 \mathrm{IL}=0 \mathrm{JL}=0 \mathrm{PI}=0 \mathrm{PJ}=0$

(9) $\mathrm{KS}=4 \mathrm{NS}=1 \mathrm{NC}=1 \mathrm{KT}=6 \mathrm{NT}=9 \mathrm{Fan}=9 \mathrm{IR}=1 \mathrm{JR}=1 \mathrm{IL}=1 \mathrm{JL}=1 \mathrm{PI}=0 \mathrm{PJ}=0$

(10) $\mathrm{KS}=2 \mathrm{NS}=10 \mathrm{NC}=1 \mathrm{KT}=6 \mathrm{NT}=10 \mathrm{Fan}=1 \mathrm{IR}=0 \mathrm{JR}=0 \mathrm{IL}=0 \mathrm{JL}=0 \mathrm{PI}=0 \mathrm{PJ}=0$

(11) $\mathrm{KS}=4 \mathrm{NS}=1 \mathrm{NC}=1 \mathrm{KT}=6 \mathrm{NT}=10 \mathrm{Fan}=9 \mathrm{IR}=1 \mathrm{JR}=1 \mathrm{IL}=1 \mathrm{JL}=1 \mathrm{PI}=0 \mathrm{PJ}=0$

(12) $\mathrm{KS}=2 \mathrm{NS}=10 \mathrm{NC}=1 \mathrm{KT}=6 \mathrm{NT}=11 \mathrm{Fan}=1 \mathrm{IR}=0 \mathrm{JR}=0 \mathrm{IL}=0 \mathrm{JL}=0 \mathrm{PI}=0 \mathrm{PJ}=0$

(13) $\mathrm{KS}=4 \mathrm{NS}=2 \mathrm{NC}=1 \mathrm{KT}=6 \mathrm{NT}=11 \mathrm{Fan}=9 \mathrm{IR}=1 \mathrm{JR}=1 \mathrm{IL}=1 \mathrm{IL}=1 \mathrm{PI}=0 \mathrm{PJ}=0$

(14) $\mathrm{KS}=2 \mathrm{NS}=10 \mathrm{NC}=1 \mathrm{KT}=6 \mathrm{NT}=12 \mathrm{Fan}=1 \mathrm{IR}=0 \mathrm{JR}=0 \mathrm{IL}=0 \mathrm{JL}=0 \mathrm{PI}=0 \mathrm{PJ}=0$

(15) $\mathrm{KS}=4 \mathrm{NS}=2 \mathrm{NC}=1 \mathrm{KT}=6 \mathrm{NT}=12 \mathrm{Fan}=9 \mathrm{IR}=1 \mathrm{JR}=1 \mathrm{IL}=1 \mathrm{JL}=1 \mathrm{PI}=0 \mathrm{PJ}=0$

Pre-Construction Connection Parameter Checking Complete 


\section{Resetting Connectivity-Related Arrays}

Next the network is constructed according to the file-supplied recipe. We remind the reader that, for both construction and processing purposes, the connecions are viewed from the "target-neuron" end. Thus, each entry in the following enumeration denotes the initiation of the process of buildin: the inputs to neurons on the indicated Target-Level/Sheet. A byproduct of this convention is that sheets which serve only as signal sources (the retina at $(1,1)$ and the keyboard at $(1,10)$, for instance) do not appear in the list.

\section{Building Inter- and Intra-Cortical Connectivity}

Target-Level/Sheet $2 / 10$

Target-Level/Sheet $4 / 1$

Target-Level/Sheet $4 / 2$

Target-Level/Sheet $6 / 9$

Target-Level/Sheet $6 / 10$

Target-Level/Sheet $6 / 11$

Target-Level/Sheet $6 / 12$

At this point, the connectivity array has been filled. Some final error checking (of a sort more conveniently done here than elsewhere) is performed before continuing with Sigmoid and Inverse Sigmoid generation.

\section{Checking for Cortical Parameter Errors}

\section{Checking for Connection Parameter Errors}

Next, the program generates, based on now established system parameters, the entries in the Sigmoid response function array and its "inverse".

\section{Generating Sigmoids}

Generating Inverse Sigmoids

Here the system computes and prints the values of a few general system describing parameters.

\section{Computing System Statistics}

Full-Sheet System Neuron Count $=181$

Source-Only Neuron Count $=136$

Source-Target Neuron Count $=45$

System-Wide Average Fanin $=26.2000$

The following reference to a grandmother region reflects a long excised characteristic of LILARTI systems and may be ignored.

Note that a Grandmother region is specified but not defined.

At this point, the NDE data file would be read if the NDE option had been invoked. The list of Screen Plot values notifies the user of the identities (in plotting order) of the 
neural sheets which will be displayed if the user selects one of the plot options. Note that these parameters can be altered at any time the NEURONS> prompt appears by responding with ADJ selecting the appropriate option.

Default Screen Plot Level/Sheet values:

Level/Sheet 4/1:

Current Neuron "N" Output

Current Neuron "DN" Output

Level/Sheet 4/2:

Current Neuron "N" Output

Current Neuron "DN" Output

Level/Sheet 6/9:

Current Neuron "N" Output

Level/Sheet 6/10:

Current Neuron "N" Output

Level/Sheet 6/11:

Current Neuron "N" Output

Level/Sheet 6/12:

Current Neuron " $\mathrm{N}$ " Output

The following is only in hopeful anticipation!

Ready to Cogitate

\section{NEURONS>}

With the appearance of the NEURONS> prompt, the system signals its readiness to begin one of the processes of which the program and the now-constructed network are capable. These are fundamentally two in number and include network training and network testing. Because the TUTOR mode of operation has been the more generally employed of the supported training methods and because its operation is not quite so transparent as is that of the TRAINING mode, we shall exhibit it in some detail. We suggest that, before the user invokes a potentially long-running training process, it will prove useful to take advantage of some of the utilities which facilitate examination of various aspects of the network. These are not graphically elegant in the manner of commercial neural network packages like NEURALWARE ${ }^{\mathrm{TM}}$ but should prove adequate.

When comfortable with the system, the new user should at last initiate the TUTOR training mode. Although the system defined by the data file LILARTYI.DAT can learn all 8111 pixel square test images in a matter of an hour or so (given a 486 PC and a 4167 Weitek coprocessor), we suggest experimenting first with a fraction of the whole as suggested by the sample listing appearing below. We continue the general scheme of explication with the interjection of notes we hope will be useful. The TUTOR mode sequence begins with the response TUTOR at the NEURONS $>$ prompt. 


\section{NEURONS > TUT}

Respond with Training Print and Plot Values.

Plot Option $(\mathbf{Y} / \mathbf{N})>\mathbf{N}$

Print Option $(\mathrm{Y} / \mathrm{N})>\mathbf{N}$

Respond with Testing Print and Plot Values.

Plot Option $(Y / N)>N$

Print Option $(\mathbf{Y} / \mathbf{N})>\mathbf{N}$

\section{Collect Statistics During Training $(\mathrm{Y} / \mathrm{N})>\mathbf{N}$}

If the user is interested in acquiring detailed information about the weight alteration statistics for the set of trainable connections, respond here with $\mathbf{Y}$, otherwise, with $\mathbf{N}$.

Enter Training Parameters as Requested:

Train on Full Image Set $(Y / N)>N$

If the user has an hour or two to spare, respond here with $\mathbf{Y}$, otherwise with $\mathbf{N}$.

\section{Train on Randomly Generated Partial Set $(\mathrm{Y} / \mathrm{N})>\mathrm{Y}$}

Unless the user has interest in the training response of the system to some specific images (which, recall, can be examined with the utility invoked by the response DNMG), the best choice here is $Y$ since it results in a training set whose members are randomly distributed across the complete set of 81 test images. Note that, had the user selected $\mathbf{N}$, he would have been required to specify a training set size and to enter a set of numbers defining the training set.

\section{Enter Training Set Fraction $>.3$}

Here the user determines the size of the training set.

\section{Training Set Will Include 24 Images.}

The program responds with an indication of the set size corresponding to the selected fraction.

\section{Number of Training Groups $>5$}

At this point, the user can exercise some control over the evolution of the training behaviour by defining the number of groups into which the complete training set will be divided. 
Initial Training Attempt Limit $>3$

The value of this parameter establishes the maximum number of training attempts to which an image will be exposed before training begins on a subsequent image. In the not unlikely event that training for an image is complete before this limit is reached, training for that image is discontinued.

\section{Maximum System Training Attempt Total Value $>1000000$}

The intent in establishing this parameter was to make it possible for the user to limit the total training effort which the system would expend on a specified training image set. We only rarely have wanted the system to halt before the completion of training and so generally set the value here to ten million. In practice, a few hundred training attempts should suffice for the 81 training images comprising the test set given reasonable values for system-defining parameters.

\section{Retraining Attempt Value $>2$}

During the TUTOR operation, retraining of partially "forgotten" images is frcquently required. The value of this parameter establishes the maximum number of these "re-training" attempts to which an image will be exposed before retesting is initiated. A value smaller than that entered for the Initial Training Attempt Limit is often adequate.

\section{Class Size $=4$}

Here the system reminds the user of the number of training images which will comprise a typical training group.

\section{Class Training Attempt Bored Value $>\mathbf{3 0 0}$}

The value of this parameter sets the limit for the number of training attempts which will be expended on a group during one group training pass. It should be noted that the system does not "give up" on a group whose members have proved so difficult, but, after going on with training for other groups ultimately reconsiders the recalcitrant one. Only if the Maximum System Training Attempt Total Value has been exceeded will the system fail to continue in its efforts to train every group. The value suggested here will not be reached for execution involving the so small a training set.

\section{Number of Training Groups is Reset to 6}

Because the user-supplied number of training groups does not evenly divide the number of training images, the system adds a training group to account for the residue.

At this point, the systems begins the training and testing operations associated with the TUTOR scheme and will continue unattended until all classes are trained or until the 
Maximum System Training Attempt Total Value is exceeded. If the user has loaded the LILARTYI.DAT file, the former result will obtain.

We reproduce here a few passages from the output produced during a TUTOR mode training session for a network defined by the file LILARTYLDAT and initiated by the above noted user responses. The first represents the initial system training behaviour.

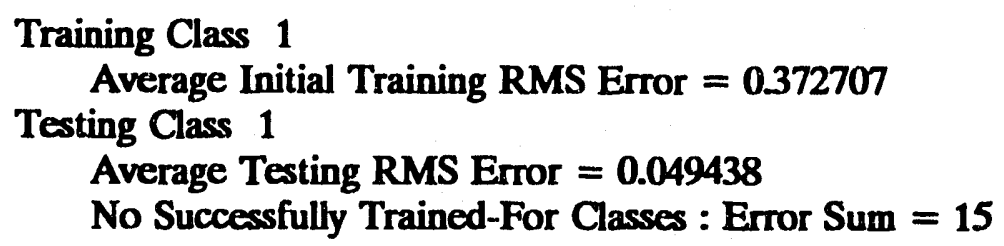

Recall from the earlier discussion of the TUTOR training mode that the general scheme involves an initial training for each class (here the operations following Training Class 1 ) followed by testing of the class for training efficacy (the operations following Testing Class 1 ). Such errors as are detected during the testing process are corrected by additional training managed by the class testing routine. If more than some minimum number of errors is detected processing continues with the first class. Otherwise, the next class is evaluated. Note that the initial training operations for a class are performed only once.

As noted earlier, RMS Error refers to the disparity obtaining at the top levels between the outputs defined by External Inter-Neural inputs and those resulting from the trainable PostSynaptic inputs. The system response, No Successfully Trained-For Classes, implies that no class has yet been processed for which no errors were detected. The alternate response, Highest \#ed Successfully Trained-For Class: n, indicates the highest training class for which the testing process has detected no errors. Error Sum is a running sum of the errors detected during class testing process and is reset each time testing and retraining restarts.

In the interest of suggesting characteristic training behaviour, we list the next six passages of output, skip and list four from roughly the middle of the training session, and, finally, jump to the last three.

Training Class 2

Average Initial Training RMS Error $=0.186163$

Testing Class 2

Average Testing RMS Error $=0.053427$

No Successfully Trained-For Classes : Error Sum $=26$

Serious errors occurred during retraining.

Processing will continue with the first training class.

Testing Class 1

Average Testing RMS Error $=0.043543$

No Successfully Trained-For Classes : Error Sum=6

Testing Class 2

Average Testing RMS Error $=\mathbf{0 . 0 4 6 3 2 4}$

No Successfully Trained-For Classes : Error Sum=10 
Serious errors occurred during retraining.

Processing will continue with the first training class.

Testing Class 1

Average Testing RMS Error $=0.030579$

No Successfully Trained-For Classes : Error Sum=2

Testing Class 2

Average Testing RMS Error $=0.033372$

No Successfully Trained-For Classes : : Error Sum=5

Serious errors occurred during retraining.

Processing will continue with the first training class.

Testing Class 1

Average Testing RMS Error $=0.023482$

No Successfully Trained-For Classes : $\quad$ Error Sum=1

Testing Class 2

Average Testing RMS Error $=0.020388$

No Successfully Trained-For Classes : $\quad$ Error Sum $=2$

Training Class 3

Average Initial Training RMS Error $=0.313475$

Testing Class 3

Average Testing RMS Error $=0.060171$

No Successfully Trained-For Classes : Error Sum=11

Serious errors occurred during retraining.

Processing will continue with the first training class.

Testing Class 1

Average Testing RMS Error $=0.042911$

No Successfully Trained-For Classes : Error Sum $=2$

Testing Class 2

Average Testing RMS Error $=0.045746$

No Successfully Trained-For Classes : Error Sum $=5$

Serious errors occurred during retraining.

Processing will continue with the first training class.

Testing Class 1

Average Testing RMS Error $=0.020208$

No Successfully Trained-For Classes : Error Sum=1

Testing Class 2

Average Testing RMS Error $=0.000000$

Highest \#ed Successfully Trained-For Class: 2 Error Sum=1 
Testing Class 3

Average Testing RMS Error $=0.039225$

Highest \#ed Successfully Trained-For Class: 2 Error Sum=5

Serious errors occurred during retraining.

Processing will continue with the first training class.

Rejoining the fray at roughly the midpoint of the training session, we find the system mastering the fifth training class for the first time and going on with the initial training of the sixth class.

\section{Testing Class 1}

Average Testing RMS Error $=0.023393$

No Successfully Trained-For Classes $\quad: 4$ Error Sum=1

Testing Class 2

Average Testing RMS Error $=0.015476$

Highest \#ed Successfully Trained-For Class: 4 Error Sum=2

Testing Class 3

Average Testing RMS Error $=0.031331$

Highest \#ed Successfully Trained-For Class: 4 Error Sum=4

Serious errors occurred during retraining.

Processing will continue with the first training class.

Testing Class 1

Average Testing RMS Error $=0.044063$

No Successfully Trained-For Classes : 4 Error Sum=1

Testing Class 2

Average Testing RMS Error $=0.000000$

Highest \#ed Successfully Trained-For Class: 4 Error Sum=1

Testing Class 3

Average Testing RMS Error $=0.000000$

Highest \#ed Successfully Trained-For Class: 4 Error Sum=1

Testing Class 4

Average Testing RMS Error $=0.023959$

Highest \#ed Successfully Trained-For Class: 4 Error Sum=2

Testing Class 5

Average Testing RMS Error $=0.000000$

Highest \#ed Successfully Trained-For Class: 5 Error Sum=2

Training Class 6

Average Initial Training RMS Error $=0.338129$

Testing Class 6

Average Testing RMS Error $=0.057518$

Highest \#ed Successfully Trained-For Class: 5 Error Sum=11

Serious errors occurred during retraining.

Processing will continue with the first training class. 
From this point to the end, the system continues to refine its behaviour until error-free testing for all classes obtains. The last three passages from the training session illustrate the last stages of the refinement process.

\section{Testing Class 1 \\ Average Testing RMS Error $=0.000000$}

No Successfully Trained-For Classes : 5 Error Sum $=0$

Testing Class 2

Average Testing RMS Error $=0.000000$

Highest \#ed Successfully Trained-For Class: 5 Error Sum=0

Testing Class 3

Average Testing RMS Error $=0.028638$

Highest \#ed Successfully Trained-For Class: 5 Error Sum=1

Testing Class 4

Average Testing RMS Error $=0.000000$

Highest \#ed Successfully Trained-For Class: 5 Error Sum=1

Testing Class 5

Average Testing RMS Error $=\mathbf{0 . 0 0 0 0 0 0}$

Highest \#ed Successfully Trained-For Class: 5 Error Sum=1

Testing Class 6

Average Testing RMS Error $=0.021181$

Highest \#ed Successfully Trained-For Class: 5 Error Sum=2

Testing Class 1

Average Testing RMS Error $=0.000000$

No Successfully Trained-For Classes : 5 Error Sum $=0$

Testing Class 2

Average Testing RMS Error $=0.026431$

Highest \#ed Successfully Trained-For Class: 5 Error Sum=1

Testing Class 3

Average Testing RMS Error $=0.000000$

Highest \#ed Successfully Trained-For Class: 5 Error Sum=1

Testing Class 4

Average Testing RMS Error $=0.000000$

Highest \#ed Successfully Trained-For Class: 5 Error Sum=1

Testing Class 5

Average Testing RMS Error $=0.000000$

Highest \# ed Successfully Trained-For Class: 5 Error Sum=1

Testing Class 6

Average Testing RMS Error $=0.000000$

Highest \#ed Successfully Trained-For Class: 6 Error Sum=1

Testing Class 1

Average Testing RMS Error $=0.000000$

No Successfully Trained-For Classes : 6 Error Sum=0

Testing Class 2

Average Testing RMS Error $=0.000000$

Highest \#ed Successfully Trained-For Class: 6 Error Sum=0 


\author{
Testing Class 3 \\ Average Testing RMS Error $=0.000000$ \\ Highest \#ed Successfully Trained-For Class: 6 Error Sum=0 \\ Testing Class 4 \\ Average Testing RMS Error $=0.000000$ \\ Highest \#ed Successfully Trained-For Class: 6 Error Sum=0 \\ Testing Class 5 \\ Average Testing RMS Error $=0.000000$ \\ Highest \#ed Successfully Trained-For Class: 6 Error Sum=0 \\ Testing Class 6 \\ Average Testing RMS Error $=0.000000$ \\ Highest \#ed Successfully Trained-For Class: 6 Error Sum=0
}

All Classes Successfully Evaluated!

\title{
NEURONS >
}

If, at the NEURONS > prompt, the user response here with LSTTUT, useful training statistics will be displayed. These include values for such parameters as Total Number of Training Passes, Total Training Time, and the like. In addition, a list of the training set image numbers and the associated numbers of training passes required for complete training appears. For the specific partial image set from which the presented results were obtained, these latter ranged from a low of 5 for image number 27 to a high of 60 for image number 23 . The average number of training passes per image over the complete 24 image training set was
20.46 .

\section{A1.2 \\ General Remarks on Constructing a Network-Defining Data File}

With the material of this section, we undertake the most difficult of the tasks this document is intended to address, the elucidation of (indeed, the first formal codification of the method of constructing a data file which the LILARTI code can interpret and express as a processable network wiring. We shall illustrate the procedure by discussing the development of the system whose wiring is encoded in the LILARTYI.DAT file, available with the Fortran source code from the authors. We note once more the already enunciated point that, when reference is made to the location of a specific neural region, the "level" and "sheet" coordinates will be enumerated (as in $(4,1)$ ). However, when discussing the characteristics (but not the position) of the same region, either of the terms, level and sheet, may be used in reference. Level and Sheet axes are assumed vertical and horizontal respectively.

We begin, as should any system constructor, by considering the task which the completed network is expected to perform. For our example, the goal was the production of a system capable of learning to describe the images in the 81 element test image set in terms of the four image-defining parameters appearing in the image-generator function (see the last material of the "Dictionary" section). This simple statement of our goal leads immediately to the definition of three regions of the required network. Since the system is to respond to images, there must be an input neural sheet at which these images can be presented. For convenience, we shall call this sheet the "Retina". Moreover, since we intend for the system 
to learn about images in terms of descriptive parameters, we must provide an input sheet at which the values of these parameters can be represented. For historical reasons, this region has come to bear the name "Keyboard Input" in the LILARTI systems. For our illustrative example, one keyboard neuron is allotted to each possible value of each parameter. Thus, because three values are permitted for each of the four parameters, the keyboard input sheet must include 12 neurons. Finally, because it will be necessary to know how the system responds to presented stimuli both during training and afterwards, we must provide for a sheet, or (more conveniently) sheets, at which the results of system processing will appear. By a perhaps unfortunately established convention, we call this region the "keyboard cortex".

Because almost all LILARTI wirings have had at least the above noted features in common, we early on adopted certain conventions concerning them. Thus, the retina is always located at level/sheet $(1,1)$, the keyboard input at $(1,10)$, and the keyboard cortex at $(6,9),(6,10)$, $(6,11)$, and $(6,12)$. The code is arranged so as to present generated images (whatever their size) at the retina at its expected location and the descriptive parameters at the keyboard input. System responses are expected at as many contiguous sheets (up to an arbitrarily defined system-set limit of 4 ) at level 6 as the user has defined. The user would be well advised to investigate the code in sufficient detail to understand the mechanisms by which these definitions are established and the ease with which they may be altered.

The system constructor will find it useful before proceeding to make a little sketch illustrating the locations and properties of the neural sheets which will comprise the completed network. To begin this sketch, dimensions for the so far established neural sheets must be determined. Although the user is free to choose as he wishes, we would suggest limiting the retinal size to 11 by 11 so as to ensure reasonably rapid processing. We note here that the code is arranged to detect retinal size and to adjust the image generation process accordingly. The keyboard input sheet at $(1,10)$ must be dimensioned so as to accommodate the number of image defining parameters and the number of values for each. These numbers are four and three respectively. Because the network construction code deals more readily with odd sheet dimensions, the keyboard input should be given the dimensions 5 by 3 (columns and rows respectively). Note that, as a result of the construction of the keyboard input loading code, any columns beyond the required four would be ignored during processing. The four keyboard-cortex sheets need be no more than 3 by 1 in order to accommodate the 81 possible parameter combinations with respect to which system training will occur.

With the making of two more system-defining decisions, we can begin the data file construction. First, we must choose the number and sizes of those intermediate sheets by which signals will be communicated from the retina to the keyboard cortex and in terms of whose mediating connections training can take place. Second, we must establish the maximum "firing rates" (the output limits) for the neurons on these sheets. For the second decision we make the simplest choice and assign a limit of 1000 to all system neurons. We note that the program generates and scales all system Sigmoid functions according to the choice or choices made for the output limit(s). Although the chosen value appears to provide adequate resolution for smooth operation, the user is free to select any value or values up to the system-permitted limit inherent in the INTEGER*2 representation of the LEVELPARM parameters. 
As may be imagined, the number and sizes of the intermediate sheets can be chosen in a variety of ways, most of which lead to networks more complex than the demands of our example problem would suggest we need. Although the simplest geometry would include but one sheet (of perhaps 25 neurons arranged in a 5 by 5 array) between the retina and the four keyboard cortex sheets, we can illustrate a bit more about system construction if we choose to include two parallel sheets, each 3 neurons square, and each mediating between the retina and but two of the four keyboard cortex neural sheets. For no particular reason, we shall establish these two mediating sheets at locations $(4,1)$ and $(4,2)$. Note that this division produces in effect two completely separate neural networks including respectively the sheets $((1,1),(4,1)$, $(6,9),(6,10))$ and $((1,1),(4,2),(6,11),(6,12))$. Although by no means critical to the current LILAR'TI scheme, we include a mediating sheet between the keyboard input and the keyboard cortex so as to ensure that neural signals reach the keyboard cortex from both sources at about the same time. This last sheet we arbitrarily locate at position $(2,10)$. The sketch of Figure 13 illustrates the locations and approximate sizes of the neural sheet distribution we wish to establish with the first entries in the data file.

ORNL-DWG 92-11944

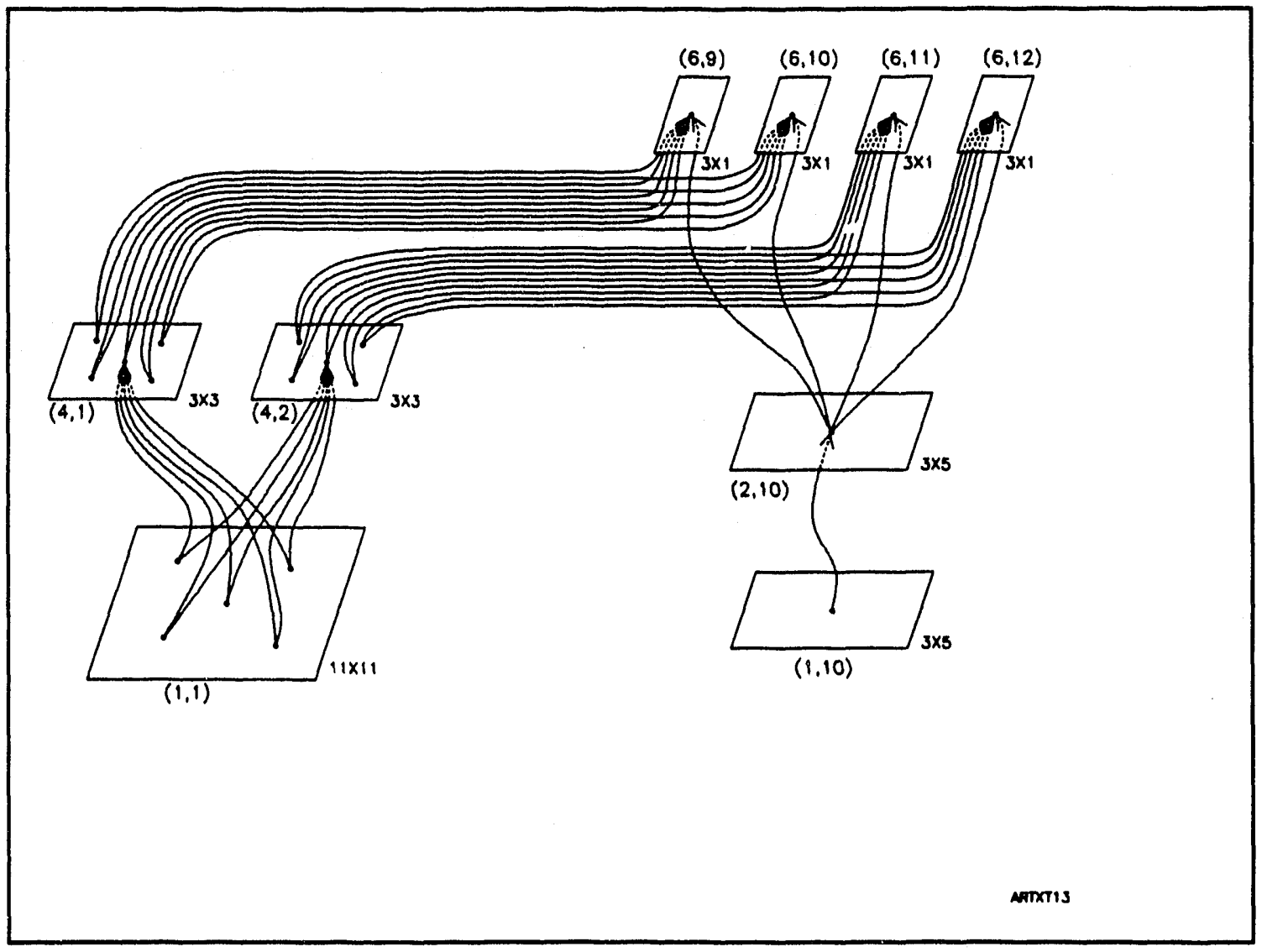

Fig. 13. Example of LILARTI network 
As one last preparation before launching into the details of file construction we review the general structure which all LILARTI parameter files must exhibit. The data in any such file are organized in four distinct sections. These are denoted in a file by the headers LEVELPARM, CORTPARM, CONNPARM, and SIGPARM. As might be supposed, entries in these four sections lead to the filling of the identically named system arrays. Within each file section the elements are separated into groups by subheaders whose forms depend upon the section type. For the LEVELPARM and SIGPARM sections the headers comprise two separated digits whose values denote the level and sheet to which the subsequent groups of data are relevant. For the CORTPARM section four digits representing source and target level/sheet pairs comprise each header. The CONNPARM section header is similar but, because provision is made that several classes of connections may link any source and target level/sheet pairs, requires one additional defining digit. Although the file-reading code could have been written in such a manner as to avoid the necessity of group terminating delimiters, their inclusion greatly simplifies matters. Thus, each group except the last within each section (regardless of section type) ends with the intentionally misspelled character string "CONNTINUE". Symbolically represented then, a LILARTI data file has the following form (note the final entry "END"):

LEVELPARM
K1 N1
DATA
CONNTINUE
K2 N2
DATA
CONNTINUE
$\vdots$
$\vdots$
Kn Nn
DATA
CORTPARM

KS1 NS1 KT1 NT1

DATA

CONNTINUE

KS2 NS2 KT2 NT2

DATA

CONNTINUE

KS3 NS3 KT3 NT3

DATA

CONNTINUE

:

KSn NSn KTn NTn

DATA

CONNPARM

NC1 KS1 NS1 KT1 NT1

DATA

CONNTINUE

NC2 KS2 NS2 KT2 NT2 


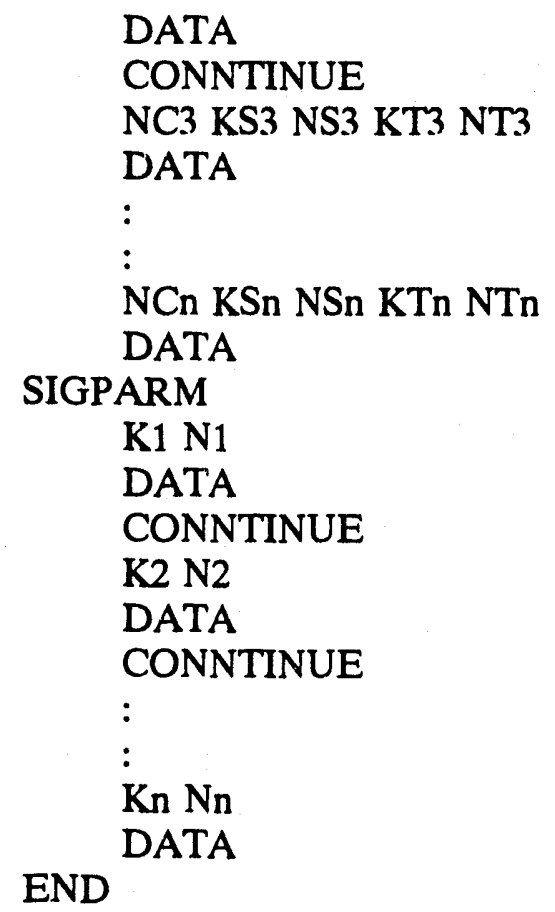

The entries in the LEVELPARM file section are intended to establish for the program just those fundamental system definitions represented by the sketch of Figure 13 and the associated text. Thus, for each level/sheet $(\mathrm{K}, \mathrm{N})$ we must include in order the Output Limit for a typical neuron on $(K, N)$, the Depth (IMAX) of $(K, N)$, and the Width (JMAX) of $(K, N)$. The Total Fanin (from all source sheets) for a typical neuron on $(K, N)$ is computed during network construction and stored in LEVELPARM $(1, \mathrm{~K}, \mathrm{~N})$. We note that this parameter serves no essential purposes in the current LILARTI and will be removed from subsequent incarnations. Observe that, because of the presence of the computed fanin parameter, there is an offset of unity between the positions of the three defining parameters in the file and in the LEVELPARM array.

We suggest that, although the file reading code is insensitive to the order in which the various level/sheet element sets are distributed, the user establish some convention for their entry as an aid to efficient file construction. Our own convention will become apparent as we proceed.

The LEVELPARM file section entries required to represent the so far described network are listed immediately below.

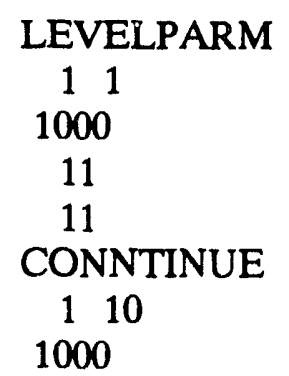




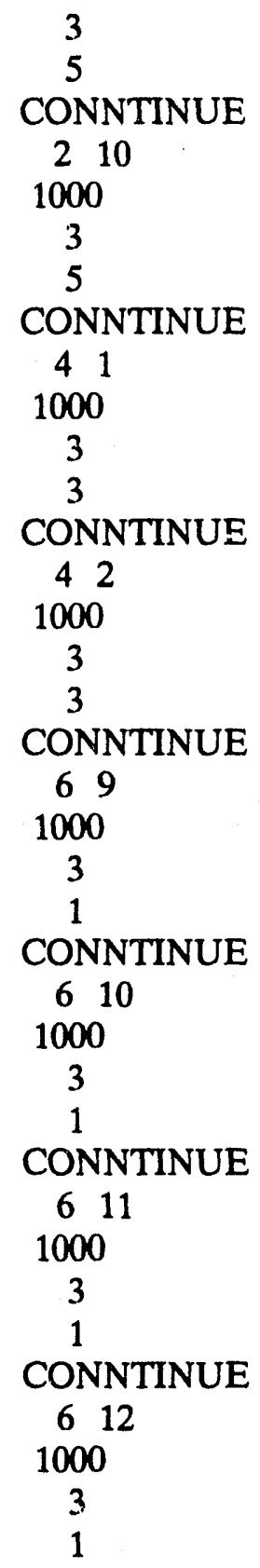

It is important to note that with the LEVELPARM entries we have described the locations and sizes of the neural regions which will comprise the completed network, but have yet described nothing about the nature of the connections which will link neurons on these regions together to form a network. Nevertheless, by this point in the file construction process, the user should have a clear picture of the general scheme of the global connectivity which is to define the completed network. In preparation for the next phase of file construction, it will prove useful to prepare a simple list representing this coarse-grained view of the system. We suggest something like the following which, here, captures the broad-scale connectivity plan suggested by Figure 13 . 
It is with the CORTPARM section of the file that we begin to represent some of the inter(and intra-) region relationships implicit in the material of Table 26. It is important to observe that, just as entries in the LEVELPARM data section led to the filling of the LEVELPARM array, file entries in the CORTPARM section lead to entries in the CORTPARM array. The most important distinction between the two types is that, whereas LEVELPARM data refer only to single regions, the entries in CORTPARM describe region pairs and define the parameters which all connections linking pairs may share (the importance of the qualifier "may" will emerge). It should be clear from these remarks that, for each of

Table 26. Coarse-grained network representation

\begin{tabular}{cc}
\hline Source region & Target region \\
\hline$(1,1)$ & $(4,1)$ \\
$(1,1)$ & $(4,2)$ \\
$(1,10)$ & $(2,10)$ \\
$(2,10)$ & $(6,9)$ \\
$(2,10)$ & $(6,10)$ \\
$(2,10)$ & $(6,11)$ \\
$(2,10)$ & $(6,12)$ \\
$(4,1)$ & $(4,1)$ \\
$(4,1)$ & $(6,9)$ \\
$(4,1)$ & $(6,10)$ \\
$(4,2)$ & $(4,2)$ \\
$(4,2)$ & $(6,11)$ \\
$(4,2)$ & $(6,12)$ \\
\hline
\end{tabular}

the source region/target region pairs of Table 26, there must be a corresponding set (or group) of entries in the CORTPARM data section. Full descriptions of the parameters which the elements of each CORTPARM group must describe can be found in the Dictionary section. By way of review, we summarize below the definitions of the nine entries which comprise each CORTPARM group. The symbols (KS,NS) and (KT,NT) refer respectively to a source region (at location (KS,NS)) and a target region (at location (KT,NT)). Note that, because the CORTPARM array fanin values (like those for the LEVELPARM array) are computed during network compilation and do not appear in the file, the file position values differ by one from the array storage positions. This first location in the CORTPARM array represents, for some source/target (KS,NS) -> (KT,NT) pair of neural regions, the Total 
Fanin for a typical neuron on (KT,NT) with respect to source neurons on (KS,NS). Although of slightly more utility than the first entry in LEVELPARM, this parameter too could be eliminated from future versions of the LILARTI code.

1. Maximum strength (magnitude) for activating connections linking (KS,NS) and (KT,NT).

2. Maximum strength (magnitude) for inhibiting connections linking (KS,NS) and (KT,NT).

3. Learning amplitude for connections linking (KS,NS) and (KT,NT).

4. Decanting parameter for connections linking (KS,NS) and (KT,NT).

5. Connection Catalyst Decrement value for activating connections linking source neurons on (KS,NS) and target neurons on (KT,NT).

6. Connection Catalyst Decrement value for inhibiting connections linking source neurons on (KS,NS) and target neurons on (KT,NT).

7. Weight Alteration Asymmetry parameter for connections linking source neurons on (KS,NS) and target neurons on (KT,NT).

8. "ALGORITHMIC" Indicator.

9. Mediating Source Sheet Parameter.

Because our illustrative network incorporates many similar regions of connectivity, we can describe the CORTPARM construction process in adequate detail without considering all inter-region combinations. If the reader has anticipated us and either looked ahead or examined the LILARTYI.DAT file, he will perhaps have noticed that some of the entries appear unnecessarily precise. Indeed, it is unlikely that any parameter is so critical of value that a change in the fifth decimal place could have a measurable effect on system operation. We include these surely unnecessary digits only as an aid to file editing since they make it possible to search for and modify multiple occurrences of but one parameter whose value is symbolically different from that of one insignificantly different numerically.

The CORTPARM file section begins with the CORTPARM header string. Following this are groups of elements each of which begins with a source region/target region designator, continues with the values of the above noted nine parameters as they apply to the designated source region/target region, and ends with the CONNTINUE or (for the last group) the CONNPARM terminator.

Upon consulting Table 26 we begin the CORTPARM section thus:

CORTPARM

$\begin{array}{llll}1 & 1 & 4 & 1\end{array}$

With the first two entries we establish the maximum values for activating and inhibiting connections linking the retina at $(1,1)$ and the mediating sheet at $(4,1)$. These we here make, except for the "editing" difference, unity. Note that both values are (and must be) positive.

Next, we set the learning rate for the $(1,1)->(4,1)$ connections. This parameter has considerable influence on the behaviour of the system and is one whose effects the user is 
urged to explore. If it is set at too large a value, training may be sufficiently compromised that the complete image set cannot be learned. For values at the other extreme, training can require unnecessarily long times.

\subsection{6}

The decanting parameter is not used in recent versions of LIL $\&$ RTI and could be set to zero for all source region/target region pairs. We list the value appearing a typical LILARTYI.DAT file for consistency.

\subsection{3}

The connection catalyst decrement values, like the learning rate, can affect system performance considerably. If the usage rate is too large, connections will become unalterable before meaningful connection patterns have been established. If it is too small these patterns may, for program versions which contain less than optimal training algorithms, never stably emerge. The values noted are serviceable but by no means optimum.

2518.01733

2508.02572

The Weight Alteration Asymmetry parameter provides a mechanism for adjusting the relative rates of activation and inhibition training. We have found that in some, as yet not fully explored, circumstances system behaviour is very dependent on the value of this parameter. Changes of one percent or so have made observable differences in system training performance.

0.99507

The "ALGORITHMIC" Indicator and Mediating Source Sheet Parameter were incorporated to support the image parsing wirings which formed such important components of the early vision-oriented LILARTI systems. Although the code for checking the validity of these parameters in the context of a complete system definition remains in the current LILARTI, code capable of utilizing them has, because our studies of eddy-current signals have taken precedence over the scene-decomposition work, been removed. Thus all current LILARTYI.DAT files are characterized by zeroes in these last two CORTPARM locations.

0.00000

0.00000

Finally, supply the group delimiter and the next source region/target region specification.

\section{CONNTINUE}

$\begin{array}{llll}1 & 1 & 4 & 2\end{array}$

Since the connections linking the retina to region $(4,2)$ are intended to form part of a network identical to the one just begun by the $(1,1) \rightarrow(4,1)$ definitions, the entries here can be identical to those just noted. 
The next five parameter groups define connections which are intended never to alter.

Further, the connections are all activating. Thus, the only non-zero parameter in each group will be the first, the maximum strength for activating connections linking the source and target regions. We list only the first group in detail.

\section{CONNTINUE \\ $\begin{array}{llll}1 & 10 & 2 & 10\end{array}$ \\ 1.00000 \\ 0.00000 \\ 0.00000 \\ 0.00000 \\ 0.00000 \\ 0.00000 \\ 0.00000 \\ 0.00000 \\ 0.00000 \\ CONNTINUE \\ 21069 \\ CONNTINUE \\ 210610 \\ CONNTINUE \\ $210 \quad 611$ \\ CONNTINUE \\ $210 \quad 6 \quad 12$}

\section{CONNTINUE}

Connections originating on region $(4,1)$ will be of two general categories, those which terminate on $(4,1)$ and those which terminate elsewhere. In this first category, there will be two classes (recall that multiple, and quite different, connection classes may link any two regions). The first will involve activating connections which link the output of each neuron to the corresponding input. The second will include nearest-neighbor inhibiting connections. The specific details of both connection classes are defined by subsequent CONNPARM entries. Since neither class will include trainable connections, we need specify here only the maximum connection strength values for the activating and inhibiting connections we anticipate.
$\begin{array}{llll}4 & 1 & 4 & 1\end{array}$
1.00000
0.99998
0.00000
0.00000
0.00000
0.00000
0.00000 


\subsection{0 \\ 0.00000 \\ CONNTINUE}

The second category of connections originating on $(4,1)$ includes the trainable links to the two keyboard regions, $(6,9)$ and $(6,10)$, which occupy the top of the first of the two independent networks. With the exception of the learning amplitude, the parameters are identical to those which have already been associated with the connections linking the retina to region $(4,1)$. The reduced learning amplitude represents an attempt to force the lower level training to codify "visual primitives" before upper level training is complete.

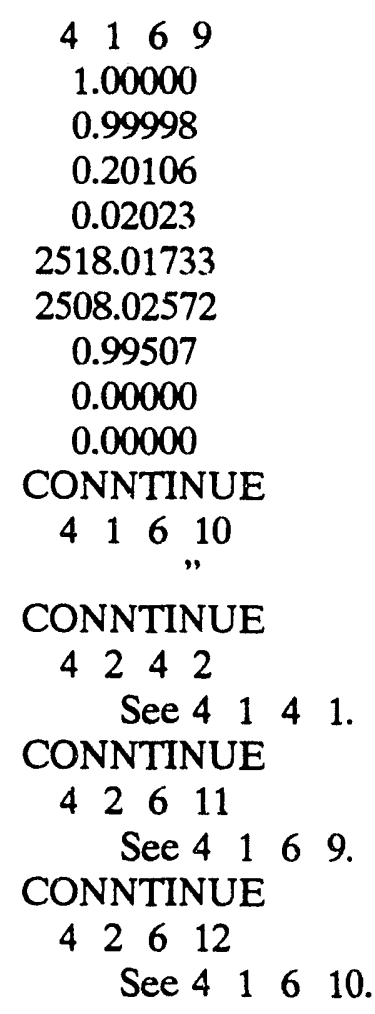

At this point in the file construction process we have defined the general or anization of a neural network but have still not defined the locus of a single connection. Indeed, in a certain sense, we shall not do so even here as we take up the details of the most complex of the four network-defining file sections. Recall that our goal in producing the very earliest LILARTI methodology was the incorporation of a "genetically based" network construction scheme which would, by crude analogy with the expression of DNA in biological systems, provide the general plan, but not the specific details of any single network. At best, we should be able to specify the characteristics of groups of connections linking two neural regions.

Lest our frequent remarks about "genetics" and the difficulty (indeed, the undesirability) of describing individual connections lead to erroneous conclusions about the nature of a LILARTI system wiring, let us here take a moment to clarify a point or two. It is certainly true that, for most of the systems which initially interested us, only the large-scale structure 
(the global properties) of the connectivity of networks was to have been definable. However, we recognized quite early that we would want to be able to define some non-trainable network regions for performing one or another image-parsing function (e.g., edge detection, vertex detection, and the like). The connectivity of these could scarcely be quasi-random in form! Thus, we arranged the connection-building code to deal in special ways with certain combinations of network-defining parameters so that, although we would still be unable to specify the coordinates of a single connection (unless source and target sheets were of unit size), we would be able to define "coherent" classes in such a manner that we would be able to predict the coordinates of all associated connections. The specific parameter combinations leading to these classes are those characterized by values for the Fanin, Range, and Periodicity for which the available source region area (in square neurons) exactly equals the fanin specified for the associated target region. When, as now, we wish to construct networks of precisely predictable connectivity, we arrange for all connectivity classes in the CONNPARM section of the parameter file to be of this special form. The connection generation code detects this form and skips the random connection generation process in favour of a much more efficient scheme.

As we have before, we shall review some of the DICTIONARY material before undertaking the next phase of file construction. Recall that each parameter group in the CONNPARM section requires five, not four, defining "coordinates". The last four of these correspond to the source region/target region parameters associated with the CORTPARM data and thus represent the implied form (KS,NS) -> (KT,NT). The first coordinate (usually denoted by "L" or "NC" in the program) identifies the connection class. Within some reasonable system limit (presently 16) there can be arbitrarily many of these so that connection distributions of many different types can link neurons on the same pair of sheets. The astute reader may recall that 21 of the 28 CONNPARM values are represented by INTEGER*2 variables in the program and are stored in the integer array ICONNPARM. The remaining 7 are stored in the floating point array CONNPARM. This distinction need not concern the network designer who should enter all 28 parameters as floating point values. For each class " $L$ " of connections linking some source region (KS,NS) to a target region (KT,NT), the following 28 definitions apply.

1. Fanin for a typical neuron on (KT,NT) with respect to source neurons on (KS,NS) for connection class $\mathrm{L}$. It is with this parameter that the designer can at last specify the number of input connections which a generic target neuron may accept (again, for the specific connection class denoted by the class index L). It should be noted that the value of this parameter must be compatible with the values of the subsequently described "Mapping" and "Range" parameters. The user may specify too few connections for the available source region area (in which case "random" connection generation will be forced) but must not specify too many. In this regard we note an important feature of the LILARTI connection building code, its connection parameter checking capability. Although it is certainly possible to construct a parameter file which will not result in a functional network, many of the file construction errors which could result in unprocessable networks are caught by the checking code. When such errors are detected, the user is notified of the error type and its position in the file.

The next two entries are the so-called "Mapping Parameters". These determine the scheme which the connection generator employs to associate the position of the center of a region of 
the source sheet (or subsheet) with the position of a neuron on a target sheet or subsheet. The acceptable values are 1.0 and 0.0 and define respectively scaled mapping and mapping with respect to source sheet midpoint (or, if one is defined, source subsheet midpoint). Under scaled mapping, simple proportion is utilized to establish, in each of the axes for which scaled mapping is specified, the center of the source region at the same fractional position in the source sheet or subsheet which the target neuron occupies in the target sheet or subsheet. Connections linking source region to target neuron are distributed in the source region with respect to this established center. Under mapping with respect to source sheet or subsheet center, all connections linking neurons in the source region to the target neuron are distributed in the source region with respect to the source region center whether the source region center is the source sheet center or a subsheet center. It is important to note that the two mapping types may be separately employed in the two sheet axes. The functions of these parameters will be clarified as we continue our illustration of the file construction process.

2. IMAP parameter for connections from (KS,NS) to (KT,NT) for connectivity class L

3. JMAP parameter for connections from $(\mathrm{KS}, \mathrm{NS})$ to $(\mathrm{KT}, \mathrm{NT})$ for connectivity class $\mathrm{L}$

The two entries, IRANGE and JRANGE, define the half dimensions of a (usually but not necessarily) small region of a source sheet (KS,NS) from which a typical target neuron on (KT,NT) can accept inputs for connectivity class $L$. The source region area defined by IRANGE and JRANGE is centered at the position defined, as noted above, by the values of IMAP and JMAP, the target neuron position, and the values of the parity parameters described below.

4. IRANGE parameter for connections from (KS,NS) to (KT,NT) for connectivity class $\mathrm{L}$

5. JRANGE parameter for connections from (KS,NS) to (KT,NT) for connectivity class $\mathrm{L}$

The "periodicity" parameters for the two coordinate axes provide for an "every Nth" source distribution. $\mathrm{N}$ is in effect the step size (which can be, and usually is, unity) for the connection building code loop which determines, within the defined source region, the identities of those neurons which will serve as sources for the current target.

6. Periodicity in "I" for connections from (KS,NS) and (KT,NT) for connectivity class L.

7. Periodicity in "J" for connections from (KS,NS) and (KT,NT) for connectivity class L.

The "parity" entries define, in effect, offsets which permit source neuron coordinates to be displaced by specified integral amounts from the position determined by the mapping parameters and the target neuron coordinates.

8. "I" parity for connections from (KS,NS) and (KT,NT) for connectivity class L.

9. "J" parity for connections from (KS,NS) and (KT,NT) for connectivity class L. 
10. (I/J) Edge-Factor. If the value of this parameter is 0 , target-to-source mapping is performed without regard to the values of the "Range" parameters. If the value is 1 , the mapping constants are computed as though the source sheet were one range parameter smaller in each dimension.

11. DUMMY (Once used for "N"/T"T"O" terminal connection indication in some of the early image-oriented LILARTI systems)

12. "EXT_I0"/POIN" Fiag. The allowed values of 0 and 1 refer respectively to connections whose target end is attached to the "External Inter-Neural" input terminal of the target neuron or to the normal, or post-synaptic input (POIN) terminal. Almost all connections in a LILARTI system will utilize the latter. It is only when the intent is to use the output of one neuron to define the "Desired N" output of another that an "EXT_I0" connection is required.

13. Self-Linking factor. If connections of some class L linking some (KS/NS) to a target region also (KS,NS) are to be of the self-linking variety, this parameter must be 1,0 otherwise. It is worth noting here that it is only because some of the earlier versions of the LILARTI code processed self-linking connections differently from those of other types that this parameter was incorporated. In general, self-linking connections are not considered trainable in the present scheme.

i

The remaining eight ICONNPARM entries permit the definition of distinct and independently functioning subsheets on both source (KS,NS) and target (KT,NT) sheets linked by connections of class $\mathrm{L}$. The first four define source subsheets, the last four target subsheets. The great utility of these last eight parameters lies in their ability to specify, between any pair of neural sheets, connectivity distributions which otherwise would have been distributed over many source sheet/target sheet pairs.

14. The extent in the I coordinate (depth) of a subsheet of a sheet (KS,NS) serving as the source for (KT,NT).

15. The extent in the $\mathrm{J}$ coordinate (width) of a subsheet of a sheet (KS,NS) serving as the source for (KT,NT).

16. The I coordinate center of the subsheet denoted by ICONNPARM(14,L,KS,NS,KT,NT) and ICONNPARM(15,L,KS,NS,KT,NT).

17. The $J$ coordinate center of the subsheet denoted by ICONNPARM(14,L,KS,NS,KT,NT) and ICONNPARM(15,L,KS,NS,KT,NT).

18. The extent in the I coordinate (depth) of a subsheet of target sheet (KT,NT) served by the source (KS,NS).

19. The extent in the $J$ coordinate (width) of a subsheet of target sheet (KT,NT) served by the source (KS,NS). 
20. The I coordinate center of the subsheet denoted by ICONNPARM(18,L,KS,NS,KT,NT) and ICONNPARM(19,L,KS,NS,KT,NT).

21. The J coordinate center of the subsheet denoted by ICONNPARM(18,L,KS,NS,KT,NT) and ICONNPARM(19,L,KS,NS,KT,NT).

The remaining seven connection-defining parameters are more conveniently expressed as decimal values. These are stored in the array, CONNPARM, whose elements are described below. The already established conventions apply. For systems likely to be of interest to the user, only the first two and the last of these seven will have non-zero values.

22. Initial Weight value. This signed parameter determines the initial connection strength for connections linking (KS,NS) and (KT,NT) for connectivity class L. Expressed as a fraction of the maximum permitted weight, the value here is multiplied by the CORTPARM parameter appearing in

CORTPARM(2,KS,NS,KT,NT) if the sign is positive and by the value appearing in CORTPARM(3,KS,NS,KT,NT) otherwise in order to establish the initial connection strength. Note that, if the system code determines from the other parameters that connections of class $L$ cannot undergo training, the initial weight value will be a system constant. The reader should bear in mind that, whereas the sign of a connection was unalterable during the training processes of earlier LILARTI systems, here a connection can take on either value. Thus, it is not unusual for a connection which was initially activating to assume an inhibiting character during the training process.

23. Local Radial Weighting exponent for connections linking source neurons on (KS,NS) and target neurons on (KT,NT) for connectivity class $\mathrm{L}$. The value appearing here determines the rate at which initial connection strengths increase (or decrease) as a function of distance from mapping point to connection origin.

24. Global Radial Weighting factor for connections linking source neurons on (KS,NS) and target neurons on (KT,NT) for connectivity class $\mathrm{L}$. For the present, a value of -1 indicates that connections originating from a point closer to an implied sheet or subsheet center than the target neuron are not constructed. A value of +1 indicates that connections originating from a point more distant from an implied sheet center than the target neuron, or equidistant from it, are not constructed. This factor was of considerable utility during the construction of certain feature extracting wirings but finds little application in present LILARTI systems.

25. Global Radial Weighting exponent for connections linking source neurons on (KS,NS) and target neurons on (KT,NT) for connectivity class L. The entry here differs from the Local Radial Weighting exponent in that it is distance from source sheet or subsheet center, rather than distance from mapping position, which is determinative.

26. Local Azimuthal Weighting exponent for connections linking source neurons on (KS,NS) and target neurons on (KT,NT) for connectivity class $\mathrm{L}$. This parameter has meaning only for sheets with an implied central origin and refers to the angle 
between the target origin and target source directions. With careful use of this parameter it is possible to construct (non-trainable) neural regions which exhibit angular sensitivity.

27. Global Azimuthal Weighting parameter for connections linking source neurons on (KS,NS) and target neurons on (KT,NT) for connectivity class L. This parameter has meaning only for sheets with an implied central origin and refers to the angle between the target-origin direction and the "Z", or vertical, direction (an assumed perpendicular joining source and target sheets). In systems of current interest to us, this parameter serves no useful purpose.

28. Initial Connection Catalyst for connections linking source neurons on (KS,NS) and target neurons on (KT,NT) for connectivity class L (expressed as a fraction of the system-wide parameter KATSYS0). Note that the rate at which a connection weight can be altered during training is proportional to the amount of connection catalyst remaining. If the value of this parameter is smaller than some system specified lower limit (KATSYSLIM0), connections of class $\mathrm{L}$ will not be altered by system training. Setting KATSYSLIM0 at some small, but non-zero, value makes possible the distinction between connections which were initially trainable, but which have exhausted their catalyst, and connections which were never trainable at all. When training has reduced the catalyst associated with a connection to the specified minimum value, the connection is thereafter treated as fixed.

We suggested earlier the utility of adopting some ordering convention for the file construction process. It will prove especially useful here to add the several sections of CONNPARM in the order already established by the CORTPARM entries. We begin, then, with the selection of parameter values for connections linking the retina at $(1,1)$ to the first intermediate sheet at $(4,1)$. The beginning of the CONNPARM section is denoted by the presence of the character string "CONNPARM". We shall require but one class of connections for the $(1,1)$ $\rightarrow>(4,1)$ linkage and can write the first two entries in the CONNPARM section thus:

\section{CONNPARM}

$\begin{array}{lllll}1 & 1 & 1 & 4 & 1\end{array}$

We note that the connection class (here denoted by the first " 1 ") could have been referred to by any number up to and including the current system limit of 16 . Although the convention of beginning each source-sheet/target-sheet pair with a class designator of "1" provides for clarity, adherence to this convention is not essential. Moreover, classes need not be entered in any particular order.

We begin by setting the fanin (relative to sheet $(1,1)$ ) for a typical neuron on sheet $(4,1)$ at 49. Recall that this implies that each target neuron on $(4,1)$ will have 49 inputs from $(1,1)$ (in addition to any others defined by subsequent $(1,1) \rightarrow(4,1)$ connection classes).

\subsection{0}

Since we shall want the inputs for each target neuron on $(4,1)$ (which, recall, is but 3 by 3 neurons square) to originate from a retinal area whose position corresponds to the position of 
the target neuron itself, we set the two mapping parameters to unity. Note that, were we to set them both to zero, the source neurons for all target neurons on $(4,1)$ would be taken from an area centered on the center of the retina. We point out that interesting connectivity patterns can be generated by setting one, but not both, of the mapping parameters to 0 when the other is unity.

\subsection{0}

1.00000

Since the retina is square (at 11 by 11), we choose for the 49 connections linking the retina to each neuron on $(4,1)$ to originate in square 7 by 7 subregions or subsheets and set both the Range parameters to 3 (since $2 * 3+1=7$ ). Note that, in the general case, the source subregion for a class can be taken as any rectangular (not necessarily square) area consistent with the class fanin value and the source sheet size.

\subsection{0}

3.00000

Because we want to accept connections from every source neuron in the source region established by the mapping parameters and the rela tive source and target sheet sizes, we set both of the periodicity parameters to unity. Note $t$ :at the parameter checking code takes the periodicity values into account in its determination of the sufficiency of source sheet space for the specified number of connections.

\subsection{0}

\subsection{0}

Since there is no point in displacing the origin of the source region from the position so far computed by the connection builder, the parity parameters can be set to zero.

\subsection{0}

0.00000

The choice we make for the (I/J) Edge-Factor, although not entirely arbitrary, is not critical to system performance. Nevertheless, because we would like for all target neurons on $(4,1)$ to have the same number of input connections from the retina, we set this parameter equal to unity. The result is that the range of positions occupied by the source mapping point is reduced by just the amount required for the extreme mapping positions to be exactly one range parameter from the retina edges. Thus, the full fanin value can still be realized. Were the (I/J) Edge-Factor set to 0, the mapping positions could approach (or include) the source sheet edges leaving area in the surrounding sheet insufficient for the origination of the full fanin complement of connections. Note that, if the mapping parameters were set to zero so that the origin of connections was the source sheet center, the (I/J) Edge-Factor would be ignored.

\subsection{0}


The value of the DUMMY parameter we set to zero. Any reader who has constructed relatively large programs and many supporting data files will appreciate our reasons for keeping this parameter, despite is present uselessness, in the file structure.

\subsection{0}

Since the connections linking the retina to $(4,1)$ must reach the normal post-synaptic input terminal, the "EXT_I0"/"POIN" Flag is set to unity.

\subsection{0}

Because connections linking two disparate neural sheets could hardly be of the self-linking variety, the Self-Linking factor is set to zero. The present version of the code should tolerate an error here.

\subsection{0}

Except for one region $((2,10)->$ All Level 6$)$ of the simple system we are illustrating, the next eight parameters for all (KS,NS) $\rightarrow(\mathrm{KT}, \mathrm{NT})$ pairs can be set to zero. So that we do not interrupt the pattern of the present presentation, we defer further commentary. The interested reader should note that, in addition to the system specific material we must provide here, additional illustrative remarks may be found in the second appendix.
0.00000
0.00000
0.00000
0.00000
0.00000
0.00000
0.00000
0.00000

For the initial weight value (or, rather, for the multiplier which determines it from the associated CORTPARM entry) we choose some small positive value. The precise figure is not of great importance, although it appears to be the case that, for two otherwise equivalent training sessions, the final configuration of a trained network wiring does depend somewhat upon the value of this parameter. Of importance here is the fact that, during the connection construction process, each computed connection weight value is "randomized" very slightly. At present, the same random sequence of strength variations is produced for each "cold start" of the connection generation process. At various times, however, the code has been arranged so as to produce different sequences for each successive system start. We were unable to detect any substantive differences between system behaviour under these two conditions.

\subsection{4}

For the Local Radial Weighting exponent we choose a value which will produce a very slight decrease in initial connection strength for connections whose originating neuron is increasingly removed from the mapping point. 
The next four parameters should be set to zero for all except those wirings specifically designed for extracting some feature or characteristic from an image.

0.00000
0.00000
0.00000
0.00000

Because we want the connections linking the retina to $(4,1)$ to be trainable, we give them the full amount of connection catalyst. Recall that the parameter value which appears here multiplies the system limit $(32000)$ to yield the value which will be stored with the generated connection(s).

$1.6000 C$

Complete the parameter group with the group terminator.

\section{CONNTINUE}

Because the connections linking the retina and $(4,2)$ are identical in form to those linking the retina to $(4,1)$, we need not describe this next group.

\section{$\begin{array}{lllll}1 & 1 & 1 & 4 & 2\end{array}$}

\section{CONNTINUE}

With the next group of connections, those linking the keyboard at $(1,10)$ to the "dummy" intermediate sheet at $(2,10)$, we illustrate a kind of connection distribution very different from those which link the retina to $(4,1)$ and to $(4,2)$. Here, since we are merely transferring signals (recall that $(2,10)$ was inserted to ensure equality of transit time for the "retinal" and "keyboard" sides of the system), we want no training to take place. Moreover, we wish to maintain strict separation of the neural pathways for each of the keyboard signals. In other words, we would like for each neuron at $(1,10)$ to link by one and only one connection to the corresponding neuron at $(2,10)$. We describe next how to establish this connectivity and observe that this case represents one of the few for which, in effect, one describes the behaviour of individual connections. Since we shall require but one class for the $(1,10) \rightarrow$ $(2,10)$ linkage, we can write the header for the group thus:

\section{$\begin{array}{lllll}1 & 1 & 10 & 2 & 10\end{array}$}

Because we shail want exactly one input connection for each neuron on $(2,10)$, we set the fanin value to unity. 
In order to ensure equivalence between the source neuron location on the source sheet $(1,10)$ and the target neuron location on the target sheet $(2,10)$, we set both Mapping parameters to unity, the Range parameters to 0 , the periodicity parameters to zero, and the parity parameters to 0 . The first condition forces proportionality between the mapping position and the target neuron position. The second in effect sets the available source region area to unity. A non-zero periodicity would be meaningless in the context of a "point" source region. The fourth condition ensures that there is no displacement between source neuron and target neuron coordinates. Note the additional requirement that for strict proportionality to be guaranteed the source and target sheets must be of the same sizes (previously established by the LEVELPARM entries as 3 by 5).
1.00000
1.00000
0.00000
0.00000
0.00000
0.00000
0.00000
0.00000

The choice we make here for the (I/J) Edge-Factor (0) is important since we want source and target sheets to be evaluated at their full sizes.

\subsection{0}

The value of the DUMMY parameter is set to zero here (and, without further comment, everywhere else as well).

\subsection{0}

Since the connections linking the keyboard to $(2,10)$ must reach the normal post-synaptic input terminal, the "EXT_IO"/"POIN" Flag is set to unity.

\subsection{0}

For the reason noted earlier, the Self-Linking factor is set to zero.

0.00000

Similarly, the next eight parameters for all $(\mathrm{KS}, \mathrm{NS}) \cdot>(\mathrm{KT}, \mathrm{NT})$ pairs can be set to zero. 
For the initial weight value (or, rather, for the multiplier which determines it from the associated CORTPARM entry) we choose a value near unity (the odd appearing digits being, as noted earlier, only for editing purposes).

0.99706

Since each target neuron bears but one input connection, the Local Radial Weighting exponent can have any value. We choose 0.

0.00000

The next four parameters, as noted earlier, will be zero for all classes.

0.00000

0.00000

0.00000

0.00000

Finally, since there is to be no training for this group of connections, the initial connection catalyst is set to zero.

\subsection{0}

CONNTINUE

With the next four groups we can illustrate two additional attributes of the LILARTI connectivity generation scheme. Of these, the first, utilization of the source subsheet parameters, is the more difficult. The other involves only the selection of the EXT_I0 (rather than the previously chosen POIN) input terminal at the target neuron. We begin by denoting the class number and the values of the source and target sheets.

\section{9}

Because again we want to maintain strict separation of the pathways for each of the signals originating at the keyboard, we set the fanin, mapping, range, periodicity, and parity values to the values already established for the connections linking the keyboard to $(2,10)$. 
For the reason noted in the context of the previous group, the (I/J) Edge-Factor and the DUMMY parameter are set to zero.

0.00000

0.00000

Because we want the outputs from $(2,10)$ to drive the "I0" inputs of the neurons at level 6 , we set the "EXT_I0"/"POIN" parameter to 0.

\subsection{0}

For the reason noted earlier, the Self-Linking factor is set to zero.

\subsection{0}

In order to illustrate the utility of both the source and target subsheet parameters, we choose an unnecessarily cumbersome method of establishing the connection distributions between $(2,10)$ and the sheets at level six (here, $(6,9))$. We remind the reader that sheet $(2,10)$ is of size 3 by 5 (rows by columns respectively) and that outputs of the neurons occupying the first four columns mimic the four active columns of the keyboard input sheet. Our goal here is to transmit the outputs from the first column to the inputs of the corresponding neurons at $(6,9)$, the outputs from the second column to the inputs of the corresponding neurons at $(6,10)$, and so forth. Noting that the first column of $(2,10)$ is of depth 3 and width 1 and is centered at row 2 , column 1 of $(2,10)$, we immediately have the four source subsheet parameters.

\subsection{0 \\ 1.00000 \\ 2.00000 \\ 1.00000}

We emphasize that, since the four target sheets at level 6 are independent, we do not have to specify target subsheets. We do so here only for illustrative purposes. If the values of the following four parameters were set to zero, the code would "assume" correctly that the entire target sheet was to be the recipient of the connections originating at the just specified subsheet of $(2,10)$.

By analogy with the definitions given for the source subsheet, we note that the target subsheet is of length 3 and width 1 and is centered at row 2 of column 1 of $(6,9)$ and immediately have values for the required parameters. 
For the connection strength, we establish a value which will lead to approximately half saturation 10 inputs at $(6,9)$.

0.52374

For the reasons already enunciated, the last six parameters must be zero.

0.00000
0.00000
0.00000
0.00000
0.00000
0.00000
CONNTINUE

The next three groups are identical to the previous one except for the source subsheet parameters. Thus, we abbreviate these group descriptions, illustrating only the differences.

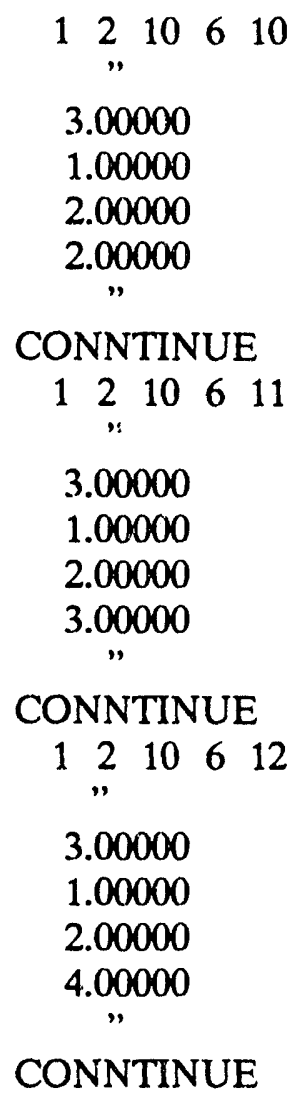

With the next two groups (and the analogous $(4,2)->(4,2)$ groups) we illustrate not only two additional connectivity types (self-linking and mutual inhibition), but the method by which to 
represent the linking of two sheets by more than one class of connections. We should remark that these two groups are included principally for illustrative purposes and that the influences of the connectivity which they represent are yet to be thoroughly evaluated. In those earlier LILARTI systems which included (in addition to the trainable regions) dozens of sheets on which only non-trainable feature extraction operations were performed, both self-activation and mutual inhibition were of vital importance. Our inclusion of these connectivity types in the present case was intended to minimize the number of neurons participating in any one stable configuration.

\section{$\begin{array}{lllll}1 & 4 & 1 & 4 & 1\end{array}$}

With the first class of $(4,1)->(4,1)$ connections we wish to establish, for each neuron on $(4,1)$, one and exactly one link between output and input. Since we require that each neuron have but one input of this class, we set the fanin value to 1 .

\subsection{0}

In order to force the source mapping point to correspond to the target neuron coordinates, we set both mapping parameters to 1 .

1.00000

1.00000

Since there must be but one possible source location in the source region (here the target neuron itself), we force the source region to have unit area by setting the range parameters to zero.

\subsection{0}

0.00000

Because periodicity cannot be defined over unit source area, the periodicity parameters are likewise set to zero.

\subsection{0}

0.00000

Since, as elsewhere noted, there should be no displacement of the origin of the source region from the position computed by the connection builder, the parity parameters can be set to zero.

\subsection{0}

0.00000

Likewise, the (I/J) Edge-Factor and the DUMMY parameters, for reasons already noted, should be zero. 
Because we want the output of a typical neuron on $(4,1)$ to contribute to the post-synaptic input of the same neuron, we set the "EXT_I0"/"POIN" parameter to 1.

1.00000

Since, with the current group of connections, we are providing for self-activation, the Self-Linking factor is set to 1.

1.00000

Since we wish to treat all neurons on the source sheet and (here the identical) target sheet equivalently, the eight source and target subsheet parameters must be zero.

0.00000

0.00000

0.00000

0.00000

0.00000

0.00000

0.00000

0.00000

We establish for the connection strength a very small value (recall that the entry here is multiplied by the corresponding CORTPARM entry to generate the weight).

0.01014

Since each target neuron for the current class bears but one input connection, the Local Radial Weighting exponent can have any value. We choose 0.

0.00000

The ccmments made elsewhere about these last five parameters apply here.

0.00000

0.00000

0.00000

0.00000

0.00000

CONNTINUE

Specification of the next connection class permits us to illustrate the method of representing multiple groups of connections linking a given source sheet/target sheet pair. This we effect by altering the class identifier index in the group header. We note again that, aside from considerations of convenience, there is no particular requirement that the classes for a given $(\mathrm{KS}, \mathrm{NS})->(\mathrm{KT} / \mathrm{NT})$ be entered in numerical order or even that the class indices be contiguous 
Because we want each neuron on $(4,1)$ to have all the others as its sources we provide for a fanin of 8 (recall that $(4,1)$ is 3 neurons square).

\subsection{0}

The requirement just noted that each neuron have all the others, rather than merely nearest neighbors, as sources forces a choice for the next two parameters different from the one so far made. Heretofore we have chosen the scaled mapping option, thus forcing the source region center to track the target neuron coordinate. We require now that each neuron on $(4,1)$ except the current target be taken as a source. By specifying the source sheet center mapping option in both axes, we guarantee that connections will be generated in just this manner. It is worth observing that, if the source subsheet parameters were non-zero, this choice of mapping parameters would force the same form of connectivity over the defined source subsheet.

0.00000

0.00000

Because we want full coverage of $(4,1)$, the range parameters must be set to unity (since $2 * 1+1=3$ ).

1.00000

1.00000

In order to ensure that every neuron on $(4,1)$ is accepted as a source, the periodicity values are both set to unity.

1.00000

1.00000

The parity values here, as elsewhere, should be zero.

0.00000

0.00000

Likewise, the (I/J) Edge-Factor and the DUMMY parameters, for reasons already noted, should be zero.

0.00000

0.00000

Because we want the output of a typical neuron on $(4,1)$ to contribute to the post-synaptic input of the target neuron, we set the "EXT_I0"/"POIN" parameter to 1. 
Since, with the current group of connections, we want specifically to avoid self-activating connections, the Self-Linking factor is set to 0 .

0.00000

As has been most frequently the case, we need not specify source or target subsheets and can thus set the next eight parameters to zero.

0.00000

0.00000

0.00000

0.00000

0.00000

0.00000

0.00000

0.00000

We establish for the connection strength a very small negative value (recall that the entry here is multiplied by the corresponding CORTPARM entry to generate the weight).

$-0.02291$

Since we require that each source contribute equally to the inhibition just specified, we set the Local Radial Weighting exponent to zero.

0.00000

The last parameters must all be zero. Note that at present the code does not support intrasheet training. Thus, there would be no effect were we to make the connection catalyst value non-zero.

0.00000

0.00000

0.00000

0.00000

0.00000

CONNTINUE

The last major groups of connections link sheets $(4,1)$ and $(4,2)$ to $(6,9),(6,10)$, and $(6,11)$, $(6,12)$ respectively. We illustrate the general scheme with the connection group linking the first intermediate sheet $(4,1)$ to the first I0-driven sheet $(6,9)$.

\section{9}

Since each target neuron on $(6,9)$ is to receive inputs from all connections on $(4,1)$ the fanin value must be 9 . 
Because we require that these nine connections span the entire source sheet $(4,1)$, we set the mapping parameters to zero. Recall that these values force the mapping point to reside at the source sheet center for all target neurons whereas, were these values unity, the connection generator would attempt to distribute the connections uniformly about a moveable mapping point which tracked the target neuron coordinates. Since $(4,1)$ is 3 by 3 , whereas $(6,9)$ is 3 by 1 , the mapping points would be (by row and column) $1 / 2,2 / 2$, and $3 / 2$ respectively for the three neurons comprising $(6,9)$.

\subsection{0}

0.00000

In order to provide sufficient (and just sufficient) area for the connections at $(4,1)$, the range parameters must be unity.

\subsection{0}

1.00000

As we have in the previous instances, we set both the periodicity parameters to unity in order to ensure that every neuron on $(4,1)$ is accepted as a source.

1.00000

1.00000

The parity parameters, as usual, should be zero.

0.00000

0.00000

Again, for reasons already noted, the (I/J) Edge-Factor and the DUMMY parameters should be zero.

0.00000

0.00000

Because we want the output of a typical neuron on $(4,1)$ to contribute to the post-synaptic input of the target neurons at $(6,9)$, we set the "EXT_I0"/"POIN" parameter to 1.

1.00000

Since the notion of self-activating connections has no meaning for disparate source and target sheets, the Self-Linking factor is set to 0 .

\subsection{0}

Since the entire source sheet is to serve as the source regions for all of $(6,9)$, we need not specify source or target subsheets and can thus set the next eight parameters to zero. 
0.00000

0.00000

0.00000

0.00000

0.00000

0.00000

0.00000

0.00000

The initial connection strength is set to some small value, the precise value of which is not of particular importance. It should be noted that there may be a difference in the final trained state configurations for two otherwise equivalent wirings whose initial connection strength parameters differ widely. This is a point not yet fully explored.

\subsection{4}

Our choice for the Local Radial Weighting exponent represents an attempt to provide an initial "bias" in the system weights. This is almost certainly unnecessary and as executed here has the unfortunate result that, for each target neuron, the source bias is centered at the source sheet midpoint.

$-0.00283$

For reasons already frequently enumerated, the next four parameters should be zero.
0.00000
0.00000
0.00000
0.00000

Because we expect training of the connections linking $(4,1)$ and $(6,9)$ we provide a full measure of connection catalyst.

\subsection{0}

\section{CONNTINUE}

The remaining five CONNPARM groups are, except for their headers, identical to ones already described. $(4,1) \rightarrow(6,10),(4,2)->(6,11)$ and $(4,2) \rightarrow(6,12)$ are identical to $(4,1) \rightarrow$ $(6,9)$. The $(4,2) \rightarrow(4,2)$ groups are identical to their corresponding $(4,1)->(4,1)$ analogues.

$\begin{array}{lllll}14 & 1 & 6 & 10\end{array}$

CONNTINUE

$\begin{array}{lllll}1 & 4 & 2 & 4 & 2\end{array}$

CONNTINUE

244242

CONNTINUE

$\begin{array}{lllll}1 & 4 & 2 & 6 & 11\end{array}$ 


\section{CONNTINUE}

$\begin{array}{lllll}1 & 4 & 2 & 6 & 12\end{array}$

We cannot hope, in this brief description of the method of constructing members of the CONNPARM data section, to provide an exhaustive (even an adequate) treatment of the full range of connectivity types which the LILARTI connection generator can produce. Indeed, we have only alluded to the quasi-random connectivity generation for which the "genetic" scheme was originally concocted. So that we do not leave this and other matters completely unattended, we treat a few of the important points in the second appendix. For the moment, however, we must turn to the description of the last of the data sections and discuss the method of specifying the Sigmoid functions which characterize the response of LILARTI neurons.

One last time, we shall review some of the DICTIONARY material before undertaking the final phase of file construction. The reader should note that, although the SIGPARM array itself has seven entries for each neural sheet, the SIGPARM file section requires, for current LILARTI systems, specification of only two. Values for any others which may be required are system derived. The elements with which the file constructor should be concerned are noted briefly below.

2. Onset input value (ONSET).

This parameter defines the input above which the output of the associated neuron will become non-zero.

3. Maximum unsaturated input (SATVAL).

The value here defines the input which results in maximum neuron output. For inputs greater than SATVAL, the output remains at the maximum value.

It was noted in the DICTIONARY section (and bears repeating here) that the just noted two parameters are stored in data files as decimal fractions and are interpreted by the program (and stored therein) in terms of the maximum pirmitted output for neurons on the sheet to which the SIGPARM file group applies. Thus, if the maximum sheet output is 1000 (a typical value) and if the ONSET parameter has the value .10000 , the stored onset value would be 100 .

5. Post-Synaptic/10 Input Error Factor.

For I0-driven neurons, the value of this parameter determines the disparity which will be tolerated between signals deriving from "trained" sources (the so-called POIN inputs) and those representing an "expected" response (the I0 inputs). Although these latter may, by suitable arrangements of interneural wiring, be injected at any point in a network, they are most commonly applied at the "top" of a "column" or "pyramid". Note that this parameter is used multiplicatively in the context of the neuron input range defined by the difference (SATVAL - ONSET).

For neurons which are not I0-driven, this parameter has a somewhat different definition.

Here, the value serves to define the amplitude by which a neuron output will be "dithered" during its attempt to discover it's optimum output value. 
The SIGPARM file section begins as have the previous two with a header occupying the position which here the "CONNTINUE" would have taken for an additional CONNPARM group. Following the header is an argument pair, identical in form to the pair encountered in the LEVELPARM section, designating the level/sheet indices for the data group to follow. Note that the file-reading code will check to ensure that there is a SIGPARM data group for every established neural sheet in the system.

\section{SIGPARM}

11

For the retina, and for the other non-trainable neural regions, a resolution value of 128 has proved more than adequate for those code versions in which the parameter had meaning. It does not at present. The entered value now is no more than a place holder.

\subsection{0}

The values given here for the onset and saturation fractions are typical of those which were employed in our first vision systems and were intended to provide a long, quasi-linear region in the Sigmoia response function. The specific values are of little consequence in the present context.

0.01500

0.98500

If the Minimum Neuron Output Limit were employed in the current code, the supplied value for the retina would be zero since approximately mid-saturation inputs are almost invariably applied to the retinal external inputs. Again, for the present code version, the parameter is unused.

0.00000

Because the neurons at the retina bear no trainable input connections, a non-zero Post-Synaptic/10 Input Error Factor would have no meaning. The value for this parameter too is set to zero.

0.00000

CONNTINUE

SIGPARM parameters for the keyboard input sheet $(1,10)$ and the transfer sheet $(2,10)$ can have values identical to those for the retina.

110

\section{CONNTINUE}

,

CONNTINUE 
For the trainable intermediate sheets $(4,1)$ and $(4,2)$ and the trainable top level sheets $(6,9)$, $(6,10),(6,11)$ and $(6,12)$ we require somewhat different behaviour for the Sigmoid response functions than that so far defined. We must preface our remarks here with the comment that we have only begun our exploration of the effects of changes in the SIGPARM parameters on system behaviour. The values listed below reflect only one of several combinations which resulted in reasonable system training behaviour. The reader is urged to experiment.

\section{1}

The entry for the Sigmoid index limit is again a place holder. For earlier versions of the code, we chose a value somewhat larger than those characteristic of the retina and keyboard. The intent was to provide for finer grained neural response in those regions whose input connections undergo training and thereby to facilitate the formation of more precisely defined trained states.

\subsection{0}

Choices for the following values of onset and saturation fractions (leading here to onset and saturation inputs of 100 and 400 respectively) were based on the requirement that the trainably connected neurons have more than unity "gain" and yet be not unduly sensitive to small changes in input signals. The experimentally inclined reader will discover that system behaviour is more dependent on the values suppliec here than on almost any other of the more than 40 so far discussed.

0.10023

0.40023

The non-zero value exhibited here for the Minimum Neuron Output Limit factor is, again, but a place holder. Indeed, it is because the behaviours of later versions of the LILARTI code were almost independent of the value of this parameter that its use was discontinued.

0.00423

The alert reader will recall our previous remarks noting that the Post-Synaptic/10 Input Error Factor can have two meanings. For neurons driven, as are those associated with $(4,1)$, by trainable connections (and thus, by connections to the POIN terminal), but not by 10 connections, the value of the Input Error Factor serves to define the amount by which the "dither" operation will displace the " $N$ " output of a neuron during the "relaxation" phase of neural processing.

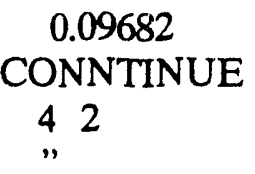

\section{CONNTINUE}

For I0-driven neurons (here, those at $(6,9),(6,10),(6,11)$, and $(6,12))$, the Post-Synaptic/10 Input Error Factor parameter serves, as its name suggests, to define an input error window. 
As such, the value of the parameter plays a very large role in the behaviour of current LILARTI systems since, in effect, it determines the amount of work which the training process must do in order to establish a set of trained states. If the value here is too large (i.e., if the tolerated error is too great), trained states will be indistinguishable and the network will have learned nothing. If the value is too small, it may prove impossible for training to produce the required number of distinct trained states. The value given here, which for the present illusitrative purposes is identical to the value entered above, results in an input error window of approximately 20 percent $(+/-\sim 10 \%$ ), a figure which (for the set of 81 system generated test images) leads to rapid training and consistent dominance of each desired state over any others. The interested reader will find it instructive to adjust the values of the parameters for the two distinct sheet groups independently.

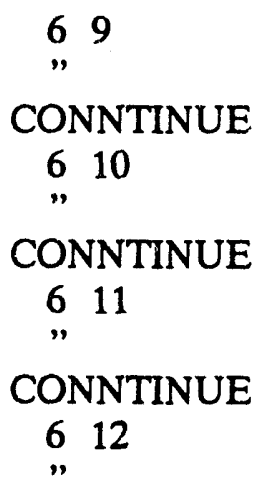

The file ends with the single string "END". An error will result if this is not present.

END

\section{Appendix A2 \\ Ilustrative Examples of Network-Defining Data Files}

In this appendix we address very briefly some aspects of the connection building process which, particularly in the discussion of the CONNPARM entries as they applied to the illustrative example network of A1.2, were of necessity slighted. Included here are sections describing the use of the source and target subsheet parameters, the function of the "lambda" parameters in constructing "periodic" connectivities, the use of multiple connection classes to create neural sheet pairs capable of finding "edges" in a presented image, and the random connection generation process. For the convenience of the reader, we separate these discussions into the four subsections which comprise this appendix.

\section{A2.1}

\section{Source- and Target-Subsheet Parameters}

The discussion of Appendix A1.2 of the CONNPARM entries for the connections linking $(2,10)$ with the four sheets at level six touched on the utility of source subsheet implementation. There the concern was the association of four successive columns of $(2,10)$ with the corresponding and distinct neural sheets $(6,9),(6,10),(6,11)$, and $(6,12)$. Although, for illustrative purposes, we used the target subsheet parameters to identify the coordinates of the target ends of the connections, we emphasized that, since the four target regions at level 6 
were independent sheets (rather than portions of one sheet), it was not, in fact, necessary to specify target subsheets. For any of the target sheets, were the values of the four target subsheet parameters set to zero, the connection generation code would "assume" correctly that the entire target sheet was to be the recipient of the connections originating at the subsheet of $(2,10)$ specified by the source subsheet parameters.

Here we shall describe the method of choosing values for the source and target subsheet parameters for a source sheet/target sheet pair each member of which will include subsheets which are to te linked in specified ways. So as to illustrate (or at the very least, suggest) some additional points, we choose an example somewhat more complicated than good pedagogy might suggest. The sketch of Figure 14 includes the salient features. Here, a 25 neuron square source sheet at $(2,1)$ is to be linked to a 20 neuron square target sheet at $(3,1)$, among other ways not shown, by connections originating at a source subsheet and terminating at two different target subsheets. For purposes of illustration, we place the scurce subsheet (whose size we shall make 7 by 5 ) at position row $=18$, column $=12$. The target subsheets we make 7 by 5 and 5 by 5 and place on the target sheet at row $=9$, column $=4$ and row $=11$, column $=16$ respectively. We wish to arrange for the source subsheet to drive the first target subsheet by non-trainable connections to the POIN terminals. In violation of the usual LILARTI convention which would exclude I0 and POIN connections linking the same neuron pair, the second target subsheet will! be driven by trainable connections at the POIN terminals and non-trainable connections at the 10 terminals. The connection classes defining these connectivities are described below.

We begin as we previously have with the class delimiter and continue with the header denoting the first connection class for connections linking a source sheet at $(2,1)$ and a target sheet at $(3,1)$.

\section{CONNTINUE}

\section{$\begin{array}{lllll}1 & 2 & 1 & 3 & 1\end{array}$}

We shall suppose that it is required that each neuron in the source subsheet drive a corresponding neuron in the first target subsheet by exactly one connection, a requirement which leads immediately to a fanin value of unity.

\subsection{0}

In order to force a unique correspondence between the source mapping point and the target neuron coordinates, we set both mapping parameters to 1 . Because the fanin value is unity, the mapping point (in the absence of non-zero parity values) will be the source neuron point as well.

\subsection{0}

1.00000

Since there must be, for each target neuron, but one allowed source position in the source region, we force the source region to have unit area by setting both range parameters to zero. 


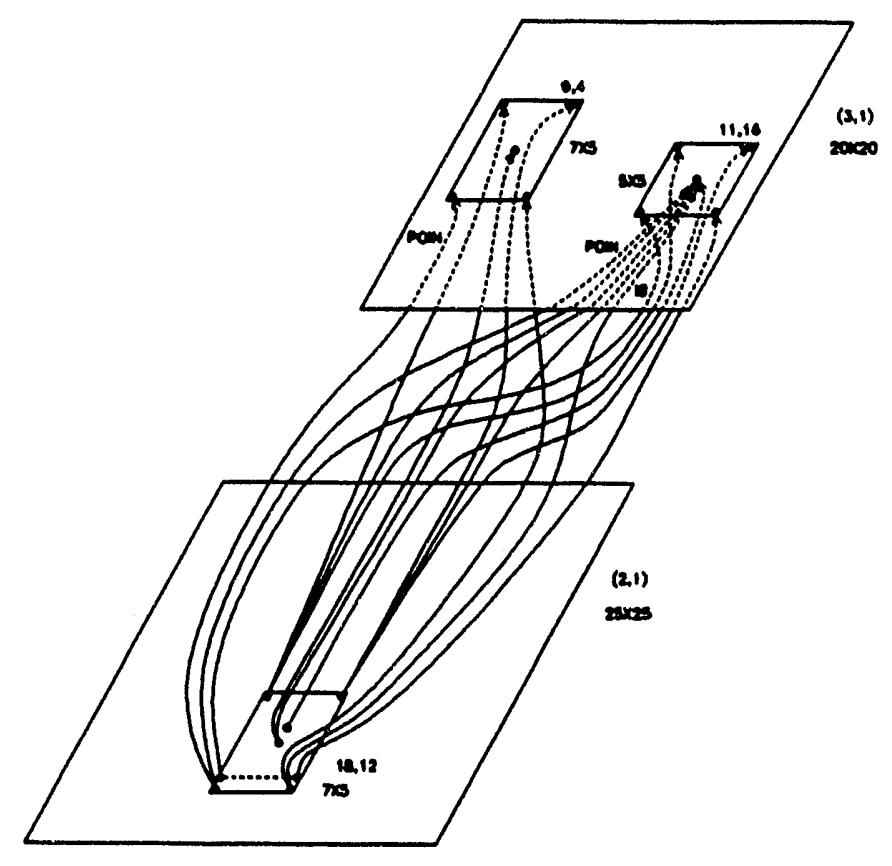

Fig. 14. Use of source and target subsheets.

Because periodicity cannot be defined over unit source area, the periodicity parameters must be zero.

\subsection{0}

0.00000

Since we require no displacement of the origin of the source region (which here is, as already noted, the position of the source neuron itself) from the position computed by the connection generator, the parity parameters should be set to zero.

\subsection{0 \\ 0.00000}

The (I/J) Edge-Factor and the DUMMY parameters, for reasons already frequently noted, should be zero. 
Because we want the output of a typical neuron on $(2,1)$ to contribute to the post-synaptic input of the target neuron, the value of the "EXT_10"/"POIN" parameter must be unity.

\subsection{0}

Since self-activation has no meaning here, the Self-Linking factor is zero.

\subsection{0}

It is with the next eight parameters that we begin our illustration of source and target subsheet definitions. Recall that these eight are divided into two groups of four, the first group defining source subsheet parameter values, the second target subsheet parameter values. With each group we specify subsheet dimensions and subsheet center coordinates. Because our illustration includes only one source subsheet, the values established here can be repeated for the subsequent connection classes. We represent the 7 row by 5 column dimensions as noted below.

Next we list the row and column coordinates of the subsheet center.

\subsection{0}

12.00000

Care must be taken with the establishment of the target subsheet parameters to ensure that we relate the desired pair of regions. With this first class, our intent is to link the source subsheet established above to the first of the target subsheets. This latter region is, like the source subsheet, 7 by 5 neurons in size and is centered at row 9, column 4. Listing these in order yields the desired entries.

It is important to appreciate the fact that with these eight entries we have defined regions of a source sheet and a target sheet which can, for almost all purposes, be treated as though they were separate neural sheets. A distinction to note with care, however, is that, whereas the sheets defined by LEVELPARM entries have a permanent "reality" (i.e., they can be accessed, displayed, etc. as independent entities), the subsheets referred to by CONNPARM entries have meaning only within the limits of the defining connection class and cannot be uniquely referenced. Nevertheless, any number of connection classes may refer to the same subsheet(s).

We establish for the connection strength a value near unity (recall that the entry here is multiplied by the corresponding CORTPARM entry to generate the weight). 
Since each target neuron for the current class bears but one input connection, the Local Radial Weighting exponent can have any value. As we have in previously noted similar cases, we choose 0 .

0.00000

The comments made elsewhere about these last five parameters apply here.

0.00000

0.00000

0.00000

0.00000

0.00000

CONNTINUE

$\begin{array}{lllll}2 & 2 & 1 & 3 & 1\end{array}$

With the second class of $(2,1) \rightarrow(3,1)$ connections we wish to establish, for each neuron in the second subsheet of $(3,1)$, links from every neuron in the subsheet of $(2,1)$. Since the source subsheet area is $35(7 \times 5)$ square neurons, the fanin value must be 35 .

35.00000

In order to force the source mapping point to be the source subsheet center, we set both mapping parameters to 0 .

0.00000

0.00000

So that the available source region space exactly matches the defined source subsheet, the range parameters must satisfy the condition:

$$
(2 * \operatorname{IRANGE}+1) *(2 * \text { JRANGE }+1)=\text { FANIN } .
$$

In the present case, the only values for IRANGE and JRANGE which satisfy this relation and which do not violate the boundaries of the source subsheet are 3 and 2 . Note that we would have chosen these values even were the source subsheet larger so as to force non-random connection generation.

3.00000

2.00000

So that all neurons in the source subsheet are taken as sources, the periodicity parameters must be unity.

1.00000

1.00000

Since we require no displacement of the origin of the source region from the position computed by the connection generator, the parity parameters must be set to zero. 
The (I/J) Edge-Factor and the DUMMY parameters, for reasons already noted, should be zero.

\subsection{0}

0.00000

Because we want the output of a typical neuron on $(2,1)$ to contribute to the post-synaptic input of the target neuron, the "EXT_I0"/"POIN" parameter must be unity.

\subsection{0}

Since self-activation has no meaning here, the Self-Linking factor is zero.

0.00000

The parameters defining the source subsheet are as previously noted.

7.00000

5.00000

18.00000

12.00000

The target subsheet for this second class is 5 rows by 5 columns in size and is centered at row 11 , column 16 of the target sheet. Listing these in order provides the target subsheet parameters.

5.00000

5.00000

11.00000

16.00000

We establish for the connection strength a value near unity (recall that the entry here is multiplied by the corresponding CORTPARM entry to generate the weight).

0.98814

As we have before, we shall use the Local Radial Weighting exponent to provide an initial radially symmetric "bias" in the system weights for the current connection class.

$-0.00283$

The comments made elsewhere about these last five parameters apply here.

0.00000

0.00000

0.00000 


\subsection{0}

0.00000

CONNTINUE

$\begin{array}{lllll}3 & 2 & 1 & 3 & 1\end{array}$

With the third class of $(2,1)->(3,1)$ connections we shall establish a completely improbable distribution of connections by linking some of the neurons in the source subsheet with corresponding neurons in the second target subsheet by $\mathrm{I} 0$ connections. We shall require that the single output from each source neuron connect to the $\mathrm{I0}$ input of the associated target neuron. A fanin value of unity is clearly implied.

\subsection{0}

In order to force the source mapping point to be uniquely associated with the position of the defining target neuron, we set both mapping parameters to 1 .

\subsection{0}

1.00000

Since there must be but one possible source location in the source subsheet for each target neuron, we force the source region to have unit area by setting the range parameters to zero.

0.00000

0.00000

The periodicity parameters, for reasons often cited, must be zero.

0.00000

0.00000

Since, as elsewhere noted, there should be no displacement of the origin of the source region from the position computed by the connection builder, the parity parameters can be set to zero.

\subsection{0}

0.00000

The (I/J) Edge-Factor and the DUMMY parameters, for reasons already noted, should be zero.

$=0.00000$

0.00000

Because we want the output of a typical neuron on $(2,1)$ to contribute to the 10 input of the target neuron, the "EXT_I0"/"POIN" parameter must be zero. 
Since self-activation has no meaning here, the Self-Linking factor is zero.

0.00000

It is with the parameters defining the source subsheet that we illustrate one last twist. We shall require that the target subsheet (which, recall, is 5 neurons square) receive inputs, not from the source subsheet as we have previously defined it, but from the 5 neuron square portion thereof occupying its "upper end". The new size we easily establish by setting the values of both source subsheet size parameters to 5 .

5.00000

5.00000

Because it is necessary only to displace the row coordinate by one in order to set the position of the new center, we have at once the entries noted below. We hasten to note that we have done nothing more with these four parameters than define an entirely new source subsheet whose neurons happen to coincide with some of those of another subsheet, a subsheet which, for connection construction purposes, will be treated entirely independently of any others.

17.00000

12.00000

The target subsheet for this third class is the area already defined and remains centered at row 11 , column 16 of the target sheet. Repeating the entries from the previous class provides the target subsheet parameters.

5.00000

5.00000

11.00000

16.00000

We establish for the connection strength a value near unity (recall that the entry here is multiplied by the corresponding CORTPARM entry to generate the weight).

0.97714

As we have noted several times, the Local Radial Weighting exponent has no meaning for classes for which the fanin is unity. Thus, we set the value of the parameter at zero.

0.00000

The comments made elsewhere about these last five parameters apply here.

0.00000

0.00000

0.00000

0.00000

0.00000 
Although with these examples we have illustrated but a few of the configurations in which source and target subsheets can exist, the range of possibilities may now be a bit more apparent. A last point, probably by now self-evident, but, because we have only alluded to it, worth explicit expression is that when source subsheets are employed the Mapping, Range, Periodicity, and Parity parameters function as though the source subsheet were in fact an ordinary sheet which happens to have the dimensions of the subsheet. This point should be born in mind when more complex connection distributions are contemplated.

\section{A2.2 \\ Periodic Connectivities}

For certain applications, it has proved useful to construct non-trainable connection distributions which are characterized by a periodicity greater than unity. We illustrate here the method of choosing CONNPARM entries so as to force the generation of a distribution in which every neuron on a target sheet (which we arbitrarily place at $(2,1)$ ) is driven at its POIN input terminal by two classes of source neurons, one class displaced by one source neuron from the other and defined by connections of equal magnitude but opposite sign with respect to the first, with the neurons of each class occupying alternate columns of a source sheet located at $(1,1)$. Although it is not required that the source sheet and target sheet be of identical size, the situation will be somewhat easier to visualize if we force this condition. For our purposes, the specific dimensions are of no consequence as long as source sheet space is provided for the "generic" connection classes. The general scheme which we wish to capture is suggested by the sketch of Figure 15.

As usual, we begin with the delimiter string and the header defining the connection class and the source sheet/target sheet pair.

\section{CONNTINUE}

$\begin{array}{lllll}1 & 1 & 1 & 2 & 1\end{array}$

Per the figure, we shall require that each target neuron be driven by five source neurons from each of the two classes, establishing the value of the fanin parameter for both classes at 5 .

\subsection{0}

In order to force the source mapping point (but not necessarily the source neuron point) to correspond uniquely to the target neuron coordinates, we set both mapping parameters to 1 .

\subsection{0}

1.00000

Since we have required five inputs per target neuron (per class) and are going to specify a periodicity of two in the "row" dimension, we must choose the values for IRANGE and JRANGE carefully. Because we want the source neurons for any target neuron to lie along a single row we must set the value of JRANGE at 0 . Because every other source neuron along the row dimension will be skipped, the value of IRANGE must be large enough to 
ORNL-DWG 92-11946

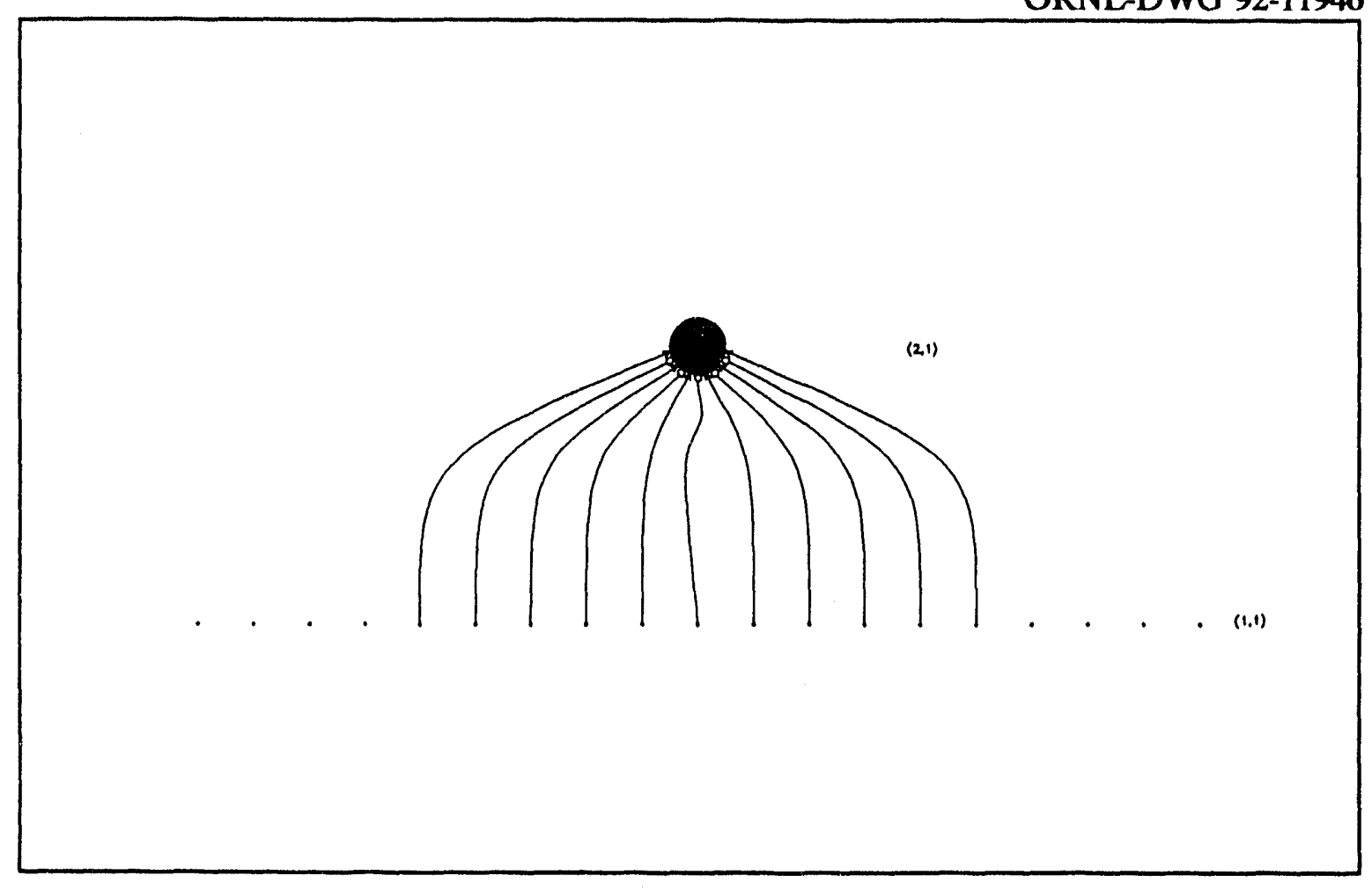

Fig. 15. Periodic connectivity.

accommodate, in effect, twice the number of source connections actually specified. Thus, IRANGE will be 4.

\subsection{0}

0.00000

To specify the "every other" source neuron distribution in the "row" dimension the row periodicity must be 2 . As often previously noted, periodicity cannot be defined over unit source area. Since the source region has unit extent along the "column" dimension, the second periodicity parameter is set to zero.

\subsection{0}

0.00000

We will require, for the first connection class, no displacement of the source region origin from the position computed by the connection generator. Thus, both parity parameters must be zero.

\subsection{0}

0.00000

The (I/J) Edge-Factor and the DUMMY parameters, for reasons frequently noted, should be zero. 
0.00000

0.00000

Because we want the output of a typical neuron on $(1,1)$ to contribute to the post-synaptic input of the target neuron on $(2,1)$, the "EXT_10"/"POIN" parameter must be unity.

1.00000

Since self-activation will not be required here, the Self-Linking factor is set to zero.

0.00000

Because we want the distribution we are defining to obtain over the entire source sheet and to involve the entire target sheet, we set all source and target subsheet parameters to zero.

0.00000

0.00000

0.00000

0.00000

0.00000

0.00000

0.00000

0.00000

We establish for the connection strength a value near unity (recall that the entry here is multiplied by the corresponding CORTPARM entry to generate the weight).

0.99914

Although it is often useful, in the context of "pre-wired" distributions, to make use of the Local Radial Weighting exponent, we set it to zero here in order to avoid further complexity.

0.00000

The comments made elsewhere about these last five parameters apply here. Recall that the fifth establishes the initial connection catalyst value. Since we want only non-learning connections for this class, the value should be 0 .

0.00000

0.00000

0.00000

0.00000

0.00000

CONNTINUE

The entries for the second class can be made by inspection if it is recalled that we require unit offset of the source neurons relative to those of the first class and that we wish to reverse the signs of the connections. We annotate only as required. 


\section{$\begin{array}{lllll}2 & 1 & 1 & 2 & 1\end{array}$}

5.00000

1.00000

1.00000

4.00000

0.00000

2.00000

0.00000

Since, for the second connection class, we require a unit displacement in the "row" coordinate of the source region origin from the position computed by the connection generator, the first parity parameter must be unity.

\subsection{0}

Since we require that the rows for the two connection classes, although offset by one neuron in the "row" coordinate, be collinear, the "column" parity parameter must be zero.

0.00000

0.00000

0.00000

1.00000

0.00000

0.00000

0.00000

0.00000

0.00000

0.00000

0.00000

0.00000

0.00000

Recalling that we want the connections of the second class to have signs opposite from those of the first, we negate the value previously entered.

$-0.99914$

0.00000

0.00000

0.00000

0.00000

0.00000

0.00000

The interested reader will find it instructive to examine the response to images of systems comprising periodic connections. Appropriately chosen combinations of periodicities in two orthogonal axes perform interesting parsing functions. 


\section{A2.3 \\ Connection Distributions for Edge-Finding}

One of the most useful image parsing functions is "edge-finding". By this term we mean to suggest, as a simple representation, an arrangement comprising a retina and an associated neural sheet of the same size as the retina whose neurons will, by the distribution of their activity, denote the presence of an edge (i.e., a contrast of at least some specified magnitude along a specified axis) in corresponding locations on the retina. We will concern ourselves here specifically with the creation of a connection distribution "tuned" to find "left" edges in a presented image. In operation, those neurons on the upper of the two illustrated sheets will respond for which the regions of the retina for which they map exhibit at least the required direction-specific contrast in the "column" coordinate (since a left or right edge is realized along a row).

With Figure 16 we illustrate both the retinal intensity variation which we wish to detect and the neural scheme by which to effect the detection. Note that what the depicted neural wiring will respond to is not just one specified contrast value, but any which exceeds some threshold value. Thus, any region of the image exhibiting at least the required contrast will be identified by active neurons on the upper level. If, instead, it is desired to locate regions of some precisely specified contrast, a more sophisticated approach must be employed. Perhaps the simplest scheme would involve construction of multiple target sheets all connected as illustrated and each "cuned" for a specific minimum contrast (a tuning which need require only respecification of the associated Sigmoid parameters). If connections are provided so that each target neuron inhibits its corresponding member on sheets representing smaller contrast values, it will be the case that, for each image position, only the target neuron representing the prevailing contrast will be active. Alternatively, an adjustable "bias" added as an input to all neurons on a single sheet can be used to identify in succession regions of decreasing contrast. In any case, the user who chooses to experiment with variations on the presented theme should note that it will be necessary to modify the LILARTI test image generator so as to reintroduce images characterized by grey-scale.

For the sake of simplicity, we depict only those source and target neurons which are required for defining the associated connection classes and remind the reader that the illustrated connectivity pattern would be replicated across the entire source sheet/target sheet pair during the connection generation process. The general approach should be apparent from the intensity and connection weight values noted in the figure. In effect, we intend for the illustrated target neuron to respond if there is at least an increase in intensity of 100 per source neuron across the neural region serving as input to the target. So that the target neuron response will be fairly "sharp", we assume a "steep" Sigmoid for which the halfsaturation input is 100 .

So that we can make an important and general point about feature extracting neural regions, we begin our discussion of the CONNPARM entries required for edge-finding with a description of the connection classes pertinent only to the upper of the two illustrated levels, the level whose neural activity will define the loci of "left-edges" in a presented retinal image. The point we wish to make is that, whatever feature a region may be designed to extract, some mechanism should be provided whereby to ensure that, of several adjacent target neurons all suggesting the presence in the underlying region of the image of the desired 


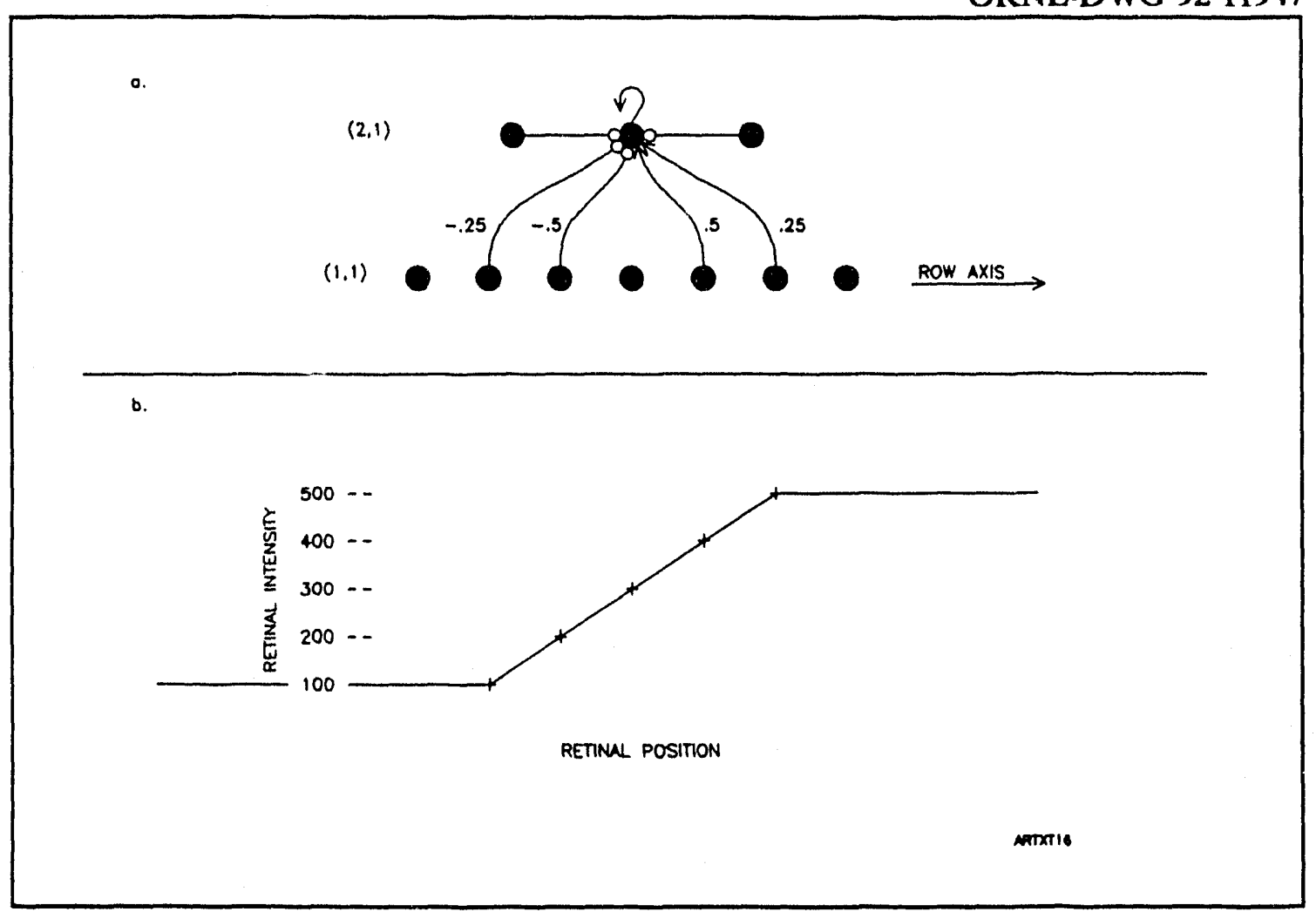

Fig. 16. Edge-finding connectivity.

feature, only the one whose input conditions are most nearly satisfied (i.e., the one most accurately representing the presence of the feature) will remain active when system stability is achieved. The required behaviour will be exhibited if target sheet neurons are connected so as to experience nearest-neighbor lateral inhibition and are provided with a small amount of positive feedback (or self-excitation). These two connectivity types will be generated by the first two connection classes noted below.

\section{CONNTINUE}

$\begin{array}{lllll}1 & 2 & 1 & 2 & 1\end{array}$

With the first of the connection classes linking $(2,1)$ to itself we define the self-activating link. Because each target neuron must receive one and only one connection of this type, the fanin value must be unity.

1.00000

In order to force the source mapping point to correspond uniquely to the target neuron coordinates (and thus, here, to ensure that the target neuron "sees" itself as the source neuron), we set both mapping parameters to 1 . 
Because we require one and only one input of the current type for each target neuron, the range parameters should detine a source area of unity. The appropriate values are 0.0 and 0.0 .

\subsection{0}

0.00000

One connection does not define a periodicity. Thus, both periodicity parameters should be zero.

\subsection{0 \\ 0.00000}

Since we require that source neuron and target neuron correspond exactly, there must be no displacement of the source region origin from the position computed by the connection generator from the target neuron coordinates.

0.00000

0.00000

The (I/J) Edge-Factor and the DUMMY parameters, for reasons frequently noted, should be zero.

0.00000

0.00000

Because we want the output of a typical neuron on $(2,1)$ to contribute to the posi-synaptic innut of the target neuron, the "EXT_I0"/"POIN" parameter must be unity.

\subsection{0}

Since we are defining a self-activating connection class here, the Self-Linking factor must be unity.

\subsection{0}

Because we want the distribution we are defining to o'stain over the entire source sheet and to involve the entire target sheet (here, of course, irentically the source sheet), we set all source and target subsheet parameters to zero. 
We establish for the self-activating connection strength a small positive value. The value of the entry here is heavily dependent on the values of several other system parameters. Both the SIGPARM parameter values and the values established for the remaining connections serving $(2,1)$ must be taken into account in selecting the most efficacious self-activation. Since we choose to avoid the additionai complexity of introducing considerations of SIGMOID parameters, we simply list a serviceable value and leave it to the reader to determine the optimum parameter values for his systems.

\subsection{7}

The Local Radial Weighting exponent should be zero as there is but one connection to consider. As we have noted elsewhere, a non-zero value would, under the circumstances, have no effect.

\subsection{0}

The comments made elsewhere about the last five parameters apply here.

0.00000

0.00000

0.00000

0.00000

0.00000

CONNTINUE

2212121

With the second of the connection classes linking $(2,1)$ to itself we define the laterally inhibiting links. In order to establish the fanin value, we must decide what we want the lateral inhibition to accomplish. If we want the effect to be the selection, over an area of the retina (the source area), of the position most representative of a "left edge", we should pick fanin and range parameters for the laterally inhibiting links so as to accept inputs from source neurons within a corresponding defining radius of the target neuron. If, instead, we want the selection process to operate only along the row occupied by the target, we should arrange for inputs only from the neurons immediately to the left and right of the target. For the purposes for which edge-finders are usually employed (among them, the reconstitution of an outline or contour map representation of a retinal image), this latter choice is the more appropriate. The fanin value should thus be set at 2 .

\subsection{0}

In order to force the source mapping point (not, ncte, the source neuron position) to correspond uniquely to the target neuron coordinates (and here, as it happens, to be coincident with the target neuron position), we set both mapping parameters to 1 . 
Because we require two inputs of the current type from source neurons distributed along the row occupied by the target neuron and immediately adjacent to it, the required "source area" is three ( 1 row by 3 columns). The range parameters are thus 1 and 0 .

\subsection{0 \\ 0.00000}

For the required connection distribution, the periodicity must be unity in the "row" coordinate and zero in the column coordinate.

\subsection{0}

0.00000

Since we require that the mapping position and the target neuron position correspond exactly, there must be no displacement of the source region origin from the position computed by the connection generator from the target neuron coordinates. Thus, the parity values are both zero.

\subsection{0}

0.00000

The $(I / J)$ Edge-Factor and the DUMMY parameters, for reasons frequently noted, should be zero.

\subsection{0}

0.00000

Because we want the output of a typical source neuron on $(2,1)$ to contribute to the postsynaptic input of the target neuron (also on $(2,1)$ ), the "EXT_I0"/"POIN" parameter must be unity.

\subsection{0}

Since self-activation is not involved here, the Self-Linking factor must be zero.

\subsection{0}

Because we want the distribution we are defining to obtain over the entire source sheet and to involve the entire target sheet, we set all source and target subsheet parameters to zero. 
We establish for the lateral inhibition connection strength a modest negative value. The intent is to make it possible for the dominant of two target neurons (i.e., the neuron with the, however slightly, greater output) to begin to extinguish its neighbors and, as a result, to be relieved of such inhibition as they had been contributing. The overall effect is one of contrast enhancement. The previous remarks concerning the importance of SIGPARM parameter values in the context of self-activation apply here as well.

$$
-0.39024
$$

Although the Local Radial Weighting exponent need not be zero here, there would be little point in making it otherwise.

\subsection{0}

The comments made elsewhere about the last five parameters apply here.

0.00000

0.00000

0.00000

0.00000

0.00000

Because we have chosen to illustrate first some points about the upper level of our two level system, we must present the final material of this section out of the order in which connection classes would normally be distributed in a connectivity file. The truly tireless reader will recall that we noted in the first appendix that the file-reading code is insensitive to the order in which classes are entered.

We begin by listing the delimiter string and the header denoting the class number and the source sheet/target sheet pair.

\section{CONNTINUE}

$\begin{array}{lllll}1 & 1 & 1 & 2 & 1\end{array}$

At first glance, it might appear that the four connections which we require for linking $(1,1)$ to $(2,1)$ could all be represented by one connection class. However, two of the four are activating connections (those served by the two source neurons on $(1,1)$ to the right of the central $(2,1)$ neuron) and two are inhibiting. Thus, since a connection class can define connections of only one sign, at least two classes will be required, one for each of the oppositely signed connection pairs originating on the two laterally displaced source neuron pairs. The astute reader will recognize that even this arrangement can only with difficulty be realized in the context of the present connection generation code because of an implicit requirement for connection class centers of symmetry. If one were willing to waste two connections, it could, for instance, be arranged that two classes of three oppositely signed connections be established, each offset by one neuron from the central source neuron (which, note, is not a source for the current target neuron). If the initial weights were made equal in magnitude and if the radial weighting factors were likewise equal, the two connections which would link the central source neuron to the target neuron would have equal and opposite 
effects. Although such an inefficient approach is a reasonable one to adopt for small systems, for larger ones for which total connection count is an important consideration, the scheme should be avoided.

By far the most straightforward, if somewhat cumbersome, representation scheme results from assigning one connection class to each of the four connections we need to define. Although somewhat wasteful of file space, the resulting connection distribution includes only the required connections. Note that, for each of the four classes we shall define, a fanin of unity is required.

\subsection{0}

In order to force the source mapping point to correspond uniquely to the target neuron coordinates, we set both mapping parameters to 1 . The reader should reflect here on the distinction between the functions of the mapping and parity parameters. As long as it is required that the source region coordinate center track the target neuron position in a coordinate (depth or width), the mapping parameter for that coordinate should be unity. This in no way implies that the source point is "equivalent" to the target neuron position, merely that for every target neuron position there is a corresponding source region coordinate center position. It is when a displacement of a source region coordinate position from the mapped position is required that a parity parameter is utilized.

\subsection{0}

1.00000

Because we require that the available source area be unity (since we are defining but one connection), both range parameters must be zero.

Since one connection can define no periodicity, the next parameters must likewise be zero.

0.00000

0.00000

Since this connection is to originate one neuron to the right of the mapping position, we require an offset of plus one in the "row" coordinate. No offset is required in the "column" value since the column coordinate of the source is not only to track the column coordinate of the target, but to be identical to it.

\subsection{0}

0.00000

The (I/J) Edge-Factor and the DUMMY parameters, for reasons frequently noted, should be zero. 
Because we want the output of a typical source neuron on $(1,1)$ to contribute to the postsynaptic input of the target neuron, the "EXT_I0"/"POIN" parameter must be unity.

1.00000

Since self-activating is not involved here, the Self-Linking factor must be zero.

0.00000

Because we want the distribution we are defining to obtain over the entire source sheet and to involve the entire target sheet, we set all source and target subsheet parameters to zero.
0.00000
0.00000
0.00000
0.00000
0.00000
0.00000
0.00000
0.00000

We establish for the strength of the first laterally displaced connection a value of .5 (primarily, because the value makes the arithmetic easy!).

0.50013

A non-zero value for the Local Radial Weighting exponent would be meaningless since the connection class contains but one connection.

0.00000

The comments made elsewhere about the last five parameters apply here.

0.00000

0.00000

0.00000

0.00000

0.00000

With the next class, we define the inhibiting connection which, with the exception of the inverted sign, is the mirror image of the connection just established. We annotate only where necessary.

\section{CONNTINUE}

$\begin{array}{lllll}2 & 1 & 1 & 2 & 1\end{array}$

1.00000

1.00000

1.00000 


\subsection{0 \\ 0.00000 \\ 0.00000 \\ 0.00000}

Since this connection is to originate one neuron to the left of the mapping position, we require an offset of minus one in the "row" coordinate. No offset is required in the "column" dimension.
$-1.00000$
0.00000
0.00000
0.00000
1.00000
0.00000
0.00000
0.00000
0.00000
0.00000
0.00000
0.00000
0.00000
0.00000

The entry here results from inverting the previously established value.
$-0.50013$
0.00000
0.00000
0.00000
0.00000
0.00000
0.00000

The last two classes are identical to the first two with the exceptions of the entries in the parity and weight positions. Again, we annotate only as necessary.

\section{CONNTINUE \\ $\begin{array}{lllll}3 & 1 & 1 & 2 & 1\end{array}$ \\ 1.00000 \\ 1.00000 \\ 1.00000 \\ 0.00000 \\ 0.00000 \\ 0.00000 \\ 0.00000}


Since this connection is to originate two neurons to the right of the source mapping position, we require an offset of plus two in the "row" coordinate. No offset is required in the "column" dimension.
2.00000
0.00000
0.00000
0.00000
1.00000
0.00000
0.00000
0.00000
0.00000
0.00000
0.00000
0.00000
0.00000
0.00000

The value of the weight here should be such that its product with the desired four-neuron source intensity difference (400) is 100 .

0.25001

0.00000

0.00000

0.00000

0.00000

0.00000

0.00000

With the next class, we define the inhibiting connection which, with the exception of the inverted sign, is the mirror image of the connection just established. We annotate only where necessary.

\section{CONNTINUE \\ $\begin{array}{lllll}4 & 1 & 1 & 2 & 1\end{array}$ \\ 1.00000 \\ 1.00000 \\ 1.00000 \\ 0.00000 \\ 0.00000 \\ 0.00000 \\ 0.00000}

Since this connection is to originate two neurons to the left of the source mapping position, we require an offset of minus two in the "row" coordinate. No offset is required in the "column" dimension. 


\section{$-2.00000$ \\ 0.00000 \\ 0.00000 \\ 0.00000 \\ 1.00000 \\ 0.00000 \\ 0.00000 \\ 0.00000 \\ 0.00000 \\ 0.00000 \\ 0.00000 \\ 0.00000 \\ 0.00000 \\ 0.00000}

The entry here results from inverting the previously established value.

-0.25001
0.00000
0.00000
0.00000
0.00000
0.00000
0.00000

We urge the reader to experiment with variations on the definitions just given. A trick we have often utilized involves the combination of outputs from edge-finders (at least one for each axis) to construct an approximate outline of the initially presented image. Because the outline can itself be neurally searched for features, a very effective image compression scheme can result in terms of which training of the modifiable portions of a network can occur with considerable efficiency.

\section{A2.4 \\ Random Connection Generation}

The Randorn Connection Generation process performs the function which its name suggests by distributing at random, for each neuron on a target sheet, a specified number of connections over the source sheet area associated with the target neuron. It is important to note that the random distribution will result only if the CONNPARM entries linking source and target sheets specify a number of connections which is numerically smaller than the source area (in square neurons) less one. As noted in Appendix A1.2, this artifice was introduced as a mechanism for supporting the generation of the predictable connection distributions which characterized the networks intended for applications like the NDE system discussed in Section V. When a random distribution is required, it is only necessary to specify the Fanin, Range and Periodicity parameters so that the available source region area is greater than the Fanin value plus one. Note that if the Periodicity parameters are both unity, the source region area is the product noted below. 


\section{Source Region Area $=(2 * \operatorname{IRANGE}+1) *(2 * \mathrm{JRANGE}+1)$}

Note too that, if either Periodicity parameter is greater than unity, the effective source region area is reduced by a fraction numerically equal to the inverse of the Periodicity parameter value. Furthermore, those connections which are generated will originate on source neurons distributed in the source region according to the values of the Periodicity parameters.

Because the code responsible for Random Connection Generation makes no use of such clever tricks as randomizable pointer tables, the process of filling the connectivity array could take a very long time in those cases for which the user-specified Fanin value was near the maximum value permitted for the random process. In order to facilitate the connection building process, the code is so arranged that only a limited effort will be expended on the attempt to find a "legal" source neuron for any single connection. Thus, it is quite possible that, for some (or several) target neurons, the total number of generated connections will be less than the user-specified Fanin value. In random systems, this should be of no great concern! 


\section{REFERENCES}

1. Two very different programs (both still resident on several laboratory machines) are important here, the OPS-5 expert system CHONSYN, and the LISP system CANONMAN. The former codified (in some versions) in a few thousand rules some of the important principles of organic chemical synthesis and could apply them to the synthesis of molecules as complex as a 20 - or 30 -carbon steroid. CANONMAN was both a learning system and a crude (but very effective) analogical asoning system which could, as it gained experience, apply reactions in environments analogous to, but distinct from, those for which it had been taught.

2. Included here are two groups of OPS-5 expert system programs, both intended for the degradation of a class of undesirable compounds. The waste conversion programs encoded both the structural transformations and the "meta-rules" required for performing effective transformation of hazardous waste-stream compounds into either less toxic or more useful compounds. The polymer degradation programs performed much the same function for waste polymer streams.

3. Design and Implementation of a Parallel Computer for Expert System

Applications, P. L. Butler, J. D. Allen, Jr., pp 638-45 in Applications of Artificial Intelligence VI, ed. Mohan M. Trivedi, SPIE.

4. Oliver Sacks, The Man Who Mistook His Wife for a Hat, Summit Books, New York, 1985.

5. Conversations with Ed Hagaman of the ORNL chemistry division about his young daughter.

6. Bela Julesz, Early Vision and Focal Attention, Reviews of Modern Physics, Vol, 63, No. 3, July, 1991.

7. For a brief description, see The Emperor's New Mind, Roger Penrose, p 386, Oxford University Press, 1989.

8. For a general introduction by one of the masters of the field, see Eye, Brain, and Vision by David H. Hubel, 1987, Scientific American Library, Distributed by W. H. Freeman and Company.

9. Neural Networks, Algorithms, Applications, and Programming Techniques, James A. Freeman/David M. Skapura, Addison-Wesley Publishing Company, 1991.

10. Ref. 9

11. For a general introduction, see chapters 11 through 14 of the Nondestructive Testing Handbook, Second Edition, Volume 4, American Society for Nondestructive Testing, 1986. 
12. Ref. 11 and W. E. Deeds, C. V. Dodd, G. W. Scott, "Computer-Aided Design of Multifrequency Eddy-Current Tests for Layered Conductors with Multiple Property Variations", ORNL/TM-6858, October 1979.

13. See the remarks of the first two endnotes.

14. For a review of some of the important points from very much a theoretical perspective, see M. A. Cohen, The Construction of Arbitrary Stable Dynamics in Nonlinear Neural Networks, Neural Networks, Vol. 5, pp. 83-103, 1992. 


\section{INTERNAL DISTRIBUTION}

1-2. Central Research Library

3. Document Reference Section

4-5. Laboratory Records Department

6. Laboratory Records, ORNL RC

7. ORNL Patent Section

8-10. M\&C Records Office

11-15. J. D. Allen, Jr.

16. G. T. Alley

17. P. L. Butler

18. D. F. Craig

19-23. C. V. Dodd

24. B. J. Eads

25. D. McDonald
26. D. J. McGuire

27. C. J. Remenyik

28-32. F. M. Schell

33. W. A. Simpson

34. G. Slaughter

35. J. O. Stiegler

36. Y. A. Chang (Consultant)

37. H. W. Foglesong (Consultant)

38. J. J. Hren (Consultant)

39. M. L. Savitz (Consultant)

40. J. G. Simon (Consultant)

41. K. E. Spear (Consultant)

\section{EXTERNAL DISTRIBUTION}

42. WRIGHT LABORATORY, WL/MLLP, Bldg. 655-Area B, WPAFB, OH 45433-6533

C. Bhagat

43. U.S. DOE, Oak Ridge Field Office, Oak Ridge, P.O. Box 2001, Oak Ridge, TN 37831-8600

Assistant Manager for Energy Research and Development

44-53. U.S. DOE OFFICE OF SCIENTIFIC AND TECHNICAL INFORMATION, Office of Information Services, P.O. Box 62, Oak Ridge, TN 37831 

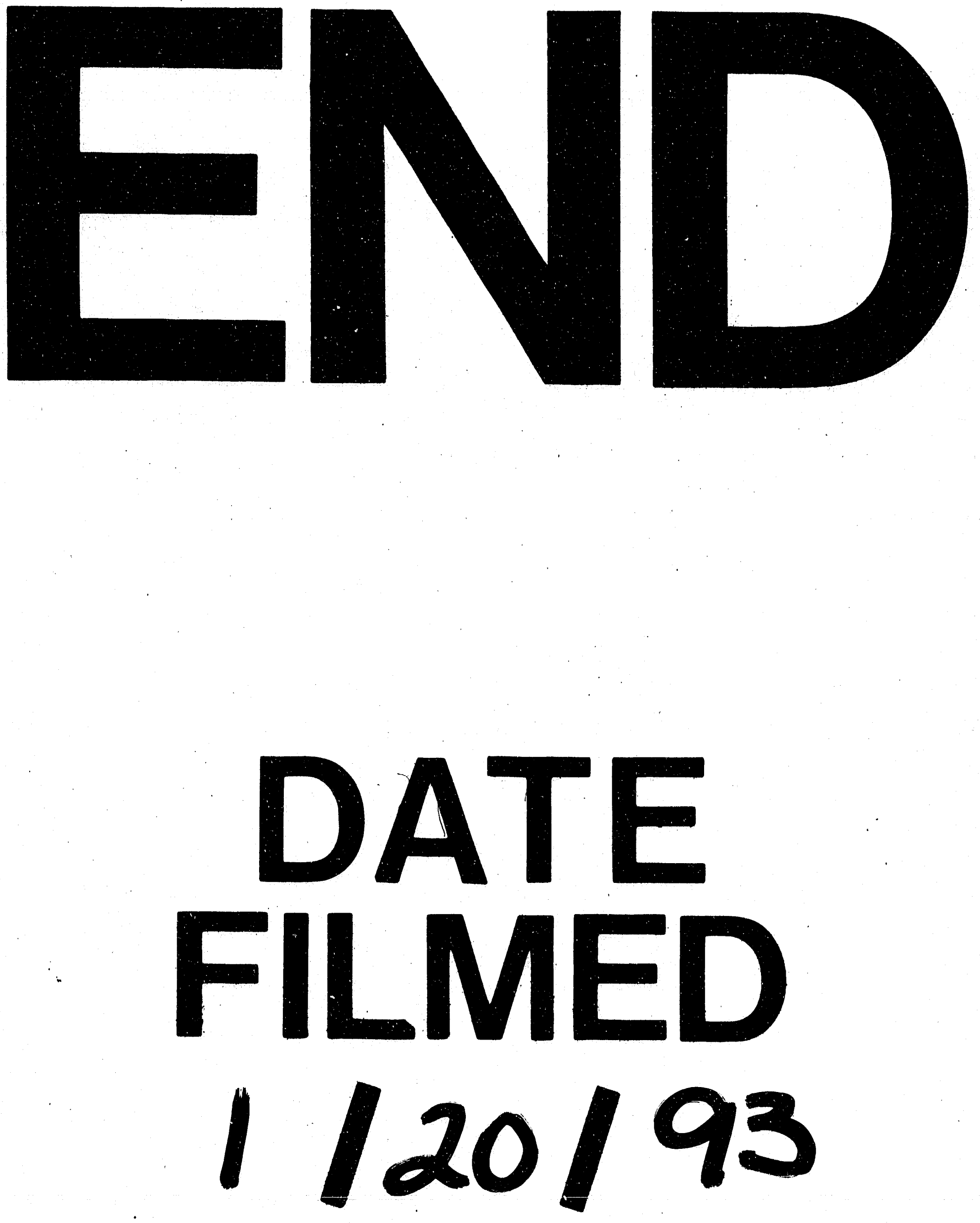

1 
